\title{
Regularity of Fourier integral operators with amplitudes in general Hörmander classes
}

\author{
Alejandro J. Castro ${ }^{1} \cdot$ Anders Israelsson ${ }^{2} \cdot$ Wolfgang Staubach ${ }^{2}$
}

Received: 21 May 2020 / Revised: 7 April 2021 / Accepted: 18 May 2021 / Published online: 5 June 2021

(C) The Author(s) 2021

\section{Abstract}

We prove the global $L^{p}$-boundedness of Fourier integral operators that model the parametrices for hyperbolic partial differential equations, with amplitudes in classical Hörmander classes $S_{\rho, \delta}^{m}\left(\mathbb{R}^{n}\right)$ for parameters $0 \leq \rho \leq 1,0 \leq \delta<1$. We also consider the regularity of operators with amplitudes in the exotic class $S_{0, \delta}^{m}\left(\mathbb{R}^{n}\right), 0 \leq \delta<1$ and the forbidden class $S_{\rho, 1}^{m}\left(\mathbb{R}^{n}\right), 0 \leq \rho \leq 1$. Furthermore we show that despite the failure of the $L^{2}$-boundedness of operators with amplitudes in the forbidden class $S_{1.1}^{0}\left(\mathbb{R}^{n}\right)$, the operators in question are bounded on Sobolev spaces $H^{s}\left(\mathbb{R}^{n}\right)$ with $s>0$. This result extends those of Y. Meyer and E. M. Stein to the setting of Fourier integral operators.

Keywords Fourier integral operators · Hyperbolic PDEs $\cdot$ Hörmander classes

Mathematics Subject Classification Primary: 42B20 - 47D06 - Secondary: 35S30 . $35 \mathrm{~L} 05$

The first author is supported by the Nazarbayev University Faculty Development Competitive Research Grants Program, grant number 110119FD4544. During the initial stage of preparation of the paper, the third author was partially supported by a grant from the Crafoord foundation.

\footnotetext{
$凶$ Wolfgang Staubach wulf@math.uu.se

Alejandro J. Castro

alejandro.castilla@nu.edu.kz

Anders Israelsson

anders.israelsson@math.uu.se

1 Department of Mathematics, Nazarbayev University, 010000 Nur-Sultan, Kazakhstan

2 Department of Mathematics, Uppsala University, S-751 06 Uppsala, Sweden
}

(Dirkhäuser 


\section{Contents}

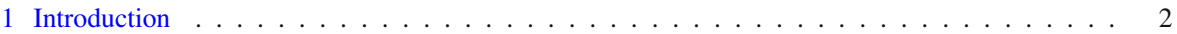

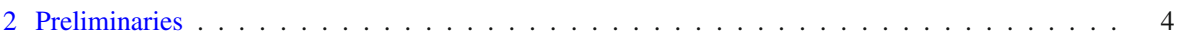

3 Reduction . . . . . . . . . . . . . . . . . . . . . 10

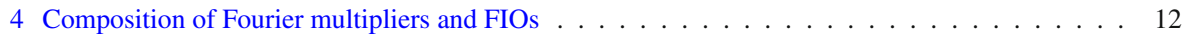

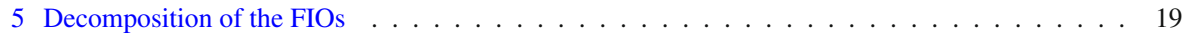

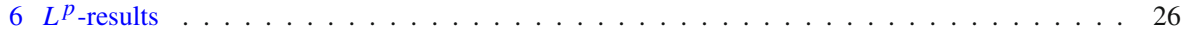

7 Sobolev space boundedness of FIOs with $s_{1,1}^{0}$-amplitudes $\ldots \ldots \ldots \ldots \ldots \ldots$

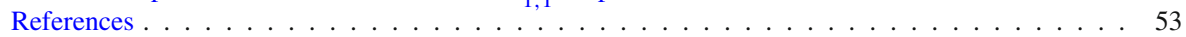

\section{Introduction}

In this paper we investigate the local and global regularity of Fourier integral operators (FIOs) of the form

$$
T_{a}^{\varphi} f(x)=\frac{1}{(2 \pi)^{n}} \int_{\mathbb{R}^{n}} e^{i \varphi(x, \xi)} a(x, \xi) \widehat{f}(\xi) \mathrm{d} \xi,
$$

with amplitudes in Hörmander classes $S_{\rho, \delta}^{m}\left(\mathbb{R}^{n}\right)$ consisting of functions in $\mathcal{C}^{\infty}\left(\mathbb{R}^{n} \times\right.$ $\mathbb{R}^{n}$ ) satisfying

$$
\left|\partial_{\xi}^{\alpha} \partial_{x}^{\beta} a(x, \xi)\right| \leq C_{\alpha, \beta}(1+|\xi|)^{m-\rho|\alpha|+\delta|\beta|}
$$

for $0 \leq \rho \leq 1,0 \leq \delta \leq 1$. More specifically we consider the boundedness in $L^{p}\left(\mathbb{R}^{n}\right)$ and $H^{s}\left(\mathbb{R}^{n}\right)$ (Sobolev spaces) for FIOs that model the parametrices of variable coefficient wave equations where the rank $\left(\partial_{\xi \xi}^{2} \varphi(x, \xi)\right)=n-1$. The corresponding investigation for the other extreme case, i.e. rank $\left(\partial_{\xi \xi}^{2} \varphi(x, \xi)\right)=0$ which is the pseudodifferential operator-case, was carried out by J. Alvarez, and J. Hounie in [1]. In that paper the authors consider the $L^{p}$-boundedness of pseudodifferential operators with symbols in $S_{\rho, \delta}^{m}\left(\mathbb{R}^{n}\right)$ where $0<\rho \leq 1,0 \leq \delta<1$. It was then shown that for these ranges of $\rho$ and $\delta$ and $1<p<\infty$ one has the $L^{p}$-boundedness of pseudodifferential operators provided that

$$
m \leq-(n-n \rho)\left|\frac{1}{p}-\frac{1}{2}\right|-n \max \left(0, \frac{\delta-\rho}{2}\right) .
$$

However, we shall also emphasize that here we are not putting any assumptions on rank $\left(\partial_{\xi \xi}^{2} \varphi(x, \xi)\right)$ in our theorems. Therefore the results that we obtain are also valid for pseudodifferential operators, although they are not necessarily optimal for these kind of operators. For instance, we obtain $L^{p}$-boundedness results for pseudodifferential operators with exotic amplitudes i.e. those with amplitudes in $S_{0, \delta}^{m}\left(\mathbb{R}^{n}\right), 0 \leq \delta<1$ (which was missing in [1]). The case of $\rho=\delta=0$ was treated (using methods that are different than ours) by R. Coifman and Y. Meyer in [5], see also [24].

The first main result of this paper regarding the $L^{p}$-boundedness of FIOs is 
Theorem 1 Let $n \geq 1,0 \leq \rho \leq 1,0 \leq \delta<1$,

$$
m=-(n-\rho)\left|\frac{1}{p}-\frac{1}{2}\right|-n \max \left(0, \frac{\delta-\rho}{2}\right),
$$

and $a \in S_{\rho, \delta}^{m}\left(\mathbb{R}^{n}\right)$. Assume that $\varphi(x, \xi) \in \mathcal{C}^{\infty}\left(\mathbb{R}^{n} \times \mathbb{R}^{n} \backslash\{0\}\right)$ is a strongly nondegenerate phase function, positively homogeneous of degree one in the frequency variable $\xi$ satisfying the following estimate

$$
\sup _{(x, \xi) \in \mathbb{R}^{n} \times \mathbb{R}^{n} \backslash\{0\}}|\xi|^{-1+|\alpha|}\left|\partial_{\xi}^{\alpha} \partial_{x}^{\beta} \varphi(x, \xi)\right| \leq C_{\alpha, \beta},
$$

for any pair of multi-indices $\alpha$ and $\beta$, with $|\alpha|+|\beta| \geq 2$. Then the FIO $T_{a}^{\varphi}$ is $L^{p}$ bounded for $1<p<\infty$.

Prior to this investigation, the only source for results regarding $L^{p}$-regularity of FIOs in $S_{\rho, \delta}^{m}$-classes were those by A. Seeger. C. Sogge and E. M. Stein [23], where the authors established the local $L^{p}$-boundedness for $\rho \in[1 / 2,1]$ and $\delta=1-\rho$.

Regarding global $L^{p}$-boundedness, the results of $\mathrm{M}$. Ruzhansky and M. Sugimoto [22] are global extensions of those of Seeger-Sogge-Stein, however they are confined to the amplitudes with $\rho=1$ and $\delta=0$.

If one goes outside the aforementioned Hörmander classes of operators, then global boundedness results have been proven in various settings for example in the papers by S. Coriasco and M. Ruzhansky [9,10] and E. Cordero, F. Nicola and L. Rodino [7,8]. Recently, A. Hassell, P. Portal and J. Rozendaal [13] obtained results regarding global boundedness of Fourier integral operators, that go beyond those in [22]. More precisely in [13] the authors also establish the regularity of FIOs with amplitudes that decay faster than those in $S_{\rho, 1-\rho}^{m}\left(\mathbb{R}^{n}\right)$ (with $\rho \in[1 / 2,1]$ ), when differentiated in the radial direction in the frequency variables. In [11] D. Dos Santos Ferreira and W. Staubach considered amplitudes in very rough classes (that also contain all the Hörmander classes $S_{\rho, \delta}^{m}\left(\mathbb{R}^{n}\right)$ ), and proved global $L^{p}$-boundedness of corresponding FIOs. However, due to the roughness of the amplitudes, the order $m$ (which depends on $\rho$ and $\delta$ ) is not as good as the expected one for smooth amplitudes, and further work is needed to achieve the right order of decay required for the $L^{p}$-boundedness of, for example, FIOs that yield parametrices for variable coefficient wave equations.

As one of the justifications of this investigation, we would like to mention that the work of R. Melrose and M. Taylor [18], and also the study of FIOs on certain nilpotent Lie groups (other than the Heisenberg group) motivates the consideration of FIOs with amplitudes in Hörmander classes $S_{1 / 3,2 / 3}^{m}\left(\mathbb{R}^{n}\right)$, for which, so far, no $L^{p}$-boundedness results have been available. Regarding $L^{2}$-boundedness of operators with general Hörmander-class amplitudes, in [11] Dos Santos Ferreira and Staubach showed that $T_{a}^{\varphi}$ is globally $L^{2}$-bounded, provided that $\rho, \delta \in[0,1], \delta \neq 1$ and $m=\min (0, n(\rho-\delta) / 2)$, or $\rho \in[0,1], \delta=1$ and $m<n(\rho-1) / 2$. This result is sharp. In this paper we also discuss the global $L^{p}$-boundedness of FIOs with forbidden amplitudes $S_{\rho, 1}^{m}\left(\mathbb{R}^{n}\right), 0 \leq \rho \leq 1$. For instance, it was shown in [11, Theorem 2.17] that FIOs with strongly non-degenerate phase functions and amplitudes in $S_{1,1}^{m}\left(\mathbb{R}^{n}\right)$ are 
$L^{p}$-bounded if and only if $m<-(n-1)|1 / p-1 / 2|$, a result which parallels the wellknown facts about pseudodifferential operators with forbidden symbols. However, the endpoint case of $S_{1,1}^{0}\left(\mathbb{R}^{n}\right)$, which is not covered by the results above, is of particular interest. Indeed as Y. Meyer [19] and E. Stein (unpublished) have shown, despite the lack of, say $L^{2}$-boundedness ([24, Prop. 2, p. 272]), the pseudodifferential operators with symbols in $S_{1,1}^{0}\left(\mathbb{R}^{n}\right)$ map Sobolev spaces $H^{s}\left(\mathbb{R}^{n}\right)$ with $s>0$ continuously to themselves. This remarkable fact has had a large impact on the applications of J. M. Bony's paradifferential calculus [2] to a systematic study of various nonlinear partial differential equations, see [25] for a comprehensive presentation. Our second main result in this paper is

Theorem 2 Let $a \in S_{1,1}^{0}\left(\mathbb{R}^{n}\right)$ and $\varphi$ satisfy the same conditions as in Theorem 1 above. Then for $s>0$ the FIO $T_{a}^{\varphi}$ is bounded from the Sobolev space $H^{s}\left(\mathbb{R}^{n}\right)$ to $H^{s}\left(\mathbb{R}^{n}\right)$.

The paper is organised as follows; in Sect. 2 we recall some definitions, facts and results from microlocal and harmonic analysis that will be used throughout the paper. In Sect. 3 we reduce the FIOs to a form that is amenable for Ruzhansky-Sugimoto's globalisation technique, which will in turn be adapted to general classes of Hörmanderclass amplitudes. In Sect. 4 we first prove a general composition formula for the left-action of a Fourier multiplier on an FIO with amplitude in general Hörmander classes. Our result extends the known results to the global setting and all values of $\rho, \delta$ (although the case of $\delta=1$ has to be excluded). Thereafter, in Sect. 5, we extend the method of Seeger-Sogge-Stein to the case of FIOs with general classical Hörmanderclass amplitudes, and decompose the Fourier integral operators into certain pieces for which we establish the basic kernel estimates. In Sect. 6 we prove Theorem 1 above (for FIOs with amplitudes in the exotic and classical Hörmander classes) as a combination of the results of Propositions 6.2 and 6.4. Finally in Sect. 7 we prove the $H^{s}$-boundedness for FIOs with amplitudes in the forbidden class $S_{1,1}^{0}\left(\mathbb{R}^{n}\right)$ for $s>0$ and thereby extend the result of Meyer and Stein to the FIO-setting. This is done by using an auxiliary class of amplitudes $C_{*}^{r} S_{1,1}^{0}\left(\mathbb{R}^{n}\right)$ which contains the class $S_{1,1}^{0}\left(\mathbb{R}^{n}\right)$, for all $r>0$ and showing that for $\max (0, r-1 / 2)<s<r$, FIOs with amplitudes in $C_{*}^{r} S_{1,1}^{0}\left(\mathbb{R}^{n}\right)$ map $H^{s}\left(\mathbb{R}^{n}\right)$ to itself.

\section{Preliminaries}

As is common practice, we will denote positive constants in the inequalities by $C$, which can be determined by known parameters in a given situation but whose value is not crucial to the problem at hand. Such parameters in this paper would be, for example, $m, p, s, n$, and the constants connected to the seminorms of various amplitudes or phase functions. The value of $C$ may differ from line to line, but in each instance could be estimated if necessary. We also write $a \lesssim b$ as shorthand for $a \leq C b$ and moreover will use the notation $a \approx b$ if $a \lesssim b$ and $b \lesssim a$.

Definition 2.1 Let $\psi_{0} \in \mathcal{C}_{c}^{\infty}\left(\mathbb{R}^{n}\right)$ be equal to 1 on $B(0,1)$ and have its support in $B(0,2)$. Then let

$$
\psi_{j}(\xi):=\psi_{0}\left(2^{-j} \xi\right)-\psi_{0}\left(2^{-(j-1)} \xi\right)
$$


where $j \geq 1$ is an integer and $\psi(\xi):=\psi_{1}(\xi)$. Then $\psi_{j}(\xi)=\psi\left(2^{-(j-1)} \xi\right)$ and one has the following Littlewood-Paley partition of unity

$$
\sum_{j=0}^{\infty} \psi_{j}(\xi)=1, \quad \text { forall } \xi \in \mathbb{R}^{n}
$$

It is sometimes also useful to define a sequence of smooth and compactly supported functions $\Psi_{j}$ with $\Psi_{j}=1$ on the support of $\psi_{j}$ and $\Psi_{j}=0$ outside a slightly larger compact set. One could for instance set

$$
\Psi_{j}:=\psi_{j+1}+\psi_{j}+\psi_{j-1}
$$

with $\psi_{-1}:=\psi_{0}$.

In what follows we define the Littlewood-Paley operators by

$$
\psi_{j}(D) f(x)=\int_{\mathbb{R}^{n}} \psi_{j}(\xi) \widehat{f}(\xi) e^{i x \cdot \xi} \mathrm{d} \xi
$$

where $₫ \xi$ denotes the normalised Lebesgue measure $\mathrm{d} \xi /(2 \pi)^{n}$ and

$$
\widehat{f}(\xi)=\int_{\mathbb{R}^{n}} e^{-i x \cdot \xi} f(x) \mathrm{d} x,
$$

is the Fourier transform of $f$. Using the Littlewood-Paley decomposition of Definition 2.1 , we define the Sobolev space $H^{s}\left(\mathbb{R}^{n}\right)$ in a somewhat unusual way. One can however show that this is equivalent to the standard definition of $H^{s}\left(\mathbb{R}^{n}\right)$.

Definition 2.2 Let $s \in \mathbb{R}$. The Sobolev space is defined by

$$
H^{s}\left(\mathbb{R}^{n}\right):=\left\{f \in \mathscr{S}^{\prime}\left(\mathbb{R}^{n}\right):\|f\|_{H^{s}\left(\mathbb{R}^{n}\right)}:=\left(\sum_{j=0}^{\infty} 4^{j s}\left\|\psi_{j}(D) f\right\|_{L^{2}\left(\mathbb{R}^{n}\right)}^{2}\right)^{1 / 2}<\infty\right\}
$$

where $\mathscr{S}^{\prime}\left(\mathbb{R}^{n}\right)$ denotes the space of tempered distributions.

Remark 2.3 Different choices of the sequence $\left\{\psi_{j}\right\}_{j=0}^{\infty}$ in Definition 2.1 give equivalent norms of $H^{s}\left(\mathbb{R}^{n}\right)$ in Definition 2.2, see e.g. [26]. We will use either $\left\{\psi_{j}\right\}_{j=0}^{\infty}$ or $\left\{\Psi_{j}\right\}_{j=0}^{\infty}$ to define the norm of $H^{s}\left(\mathbb{R}^{n}\right)$.

Remark 2.4 By Fubini's theorem, one can change the order of the norms in Definition 2.2, i.e.

$$
\|f\|_{H^{s}\left(\mathbb{R}^{n}\right)} \approx\left\|\left\{\sum_{j=0}^{\infty} 4^{j s}\left|\psi_{j}(D) f\right|^{2}\right\}^{1 / 2}\right\|_{L^{2}\left(\mathbb{R}^{n}\right)} \approx\left(\sum_{j=0}^{\infty} 4^{j s}\left\|\psi_{j}(D) f\right\|_{L^{2}\left(\mathbb{R}^{n}\right)}^{2}\right)^{1 / 2} .
$$


Also, using fairly standard Littlewood-Paley theory one can show the following well-known result:

Lemma 2.5 Let $\left\{f_{j}\right\}_{j=0}^{\infty} \subset \mathscr{S}^{\prime}\left(\mathbb{R}^{n}\right)$ be such that

$$
\operatorname{supp} \widehat{f_{j}} \subseteq\left\{\xi \in \mathbb{R}^{n}:|\xi| \lesssim 2^{j}\right\}, \quad j \geq 0
$$

Then, for $s>0$, one has

$$
\left\|\sum_{j=0}^{\infty} f_{j}\right\|_{H^{s}\left(\mathbb{R}^{n}\right)} \lesssim\left\|\left\{\sum_{j=0}^{\infty} 4^{j s}\left|f_{j}\right|^{2}\right\}^{1 / 2}\right\|_{L^{2}\left(\mathbb{R}^{n}\right)},
$$

For a proof, see e.g. [25].

In proving the $L^{p}$-boundedness of FIOs $(1<p<\infty)$, the standard procedure is to first show the boundedness of the operator (and its adjoint) from the Hardy space $\mathscr{H}^{1}\left(\mathbb{R}^{n}\right)$ to $L^{1}\left(\mathbb{R}^{n}\right)$ and thereafter interpolate the results with the $L^{2}$-boundedness. In proving the Hardy space boundedness, the main tool is to use the so-called Hardy space atoms.

Definition 2.6 Let $p \in(0,1]$. A function $\mathfrak{a}$ is called an $\mathscr{H}^{p}$-atom if for some $x_{0} \in \mathbb{R}^{n}$ and $r>0$ the following three conditions are satisfied:

(i) supp a $\subset B\left(x_{0}, r\right)$,

(ii) $|\mathfrak{a}(x)| \leq\left|B\left(x_{0}, r\right)\right|^{-1 / p}$,

(iii) $\int_{\mathbb{R}^{n}} x^{\alpha} \mathfrak{a}(x) \mathrm{d} x=0$ for all $|\alpha| \leq N$ for some $N \geq n(1 / p-1)$.

Then a distribution $f \in \mathscr{H}^{p}\left(\mathbb{R}^{n}\right)$, has an atomic decomposition

$$
f=\sum_{j=0}^{\infty} \lambda_{j} \mathfrak{a}_{j}
$$

where the $\lambda_{j}$ are constants with

$$
\|f\|_{\mathscr{H}^{p}\left(\mathbb{R}^{n}\right)}:=\inf \left(\sum_{j}\left|\lambda_{j}\right|^{p}\right)^{1 / p}<\infty .
$$

and the $\mathfrak{a}_{j}$ are $\mathscr{H}^{p}$-atoms.

Remark 2.7 Different choices of $N$ in (iii) above give equivalent definitions of the $\mathscr{H}^{p}$-norm.

Next we define the building blocks of the FIOs and the pseudodifferential operators. These are the amplitudes (symbols in the pseudodifferential setting) and the phase functions. The class of amplitudes considered in this paper were first introduced by L. Hörmander in [14]. 
Definition 2.8 Let $m \in \mathbb{R}$ and $\rho, \delta \in[0,1]$. An amplitude (symbol) $a(x, \xi)$ in the class $S_{\rho, \delta}^{m}\left(\mathbb{R}^{n}\right)$ is a function $a \in \mathcal{C}^{\infty}\left(\mathbb{R}^{n} \times \mathbb{R}^{n}\right)$ that verifies the estimate

$$
\left|\partial_{\xi}^{\alpha} \partial_{x}^{\beta} a(x, \xi)\right| \lesssim\langle\xi\rangle^{m-\rho|\alpha|+\delta|\beta|}
$$

for all multi-indices $\alpha$ and $\beta$ and $(x, \xi) \in \mathbb{R}^{n} \times \mathbb{R}^{n}$, where $\langle\xi\rangle:=\left(1+|\xi|^{2}\right)^{1 / 2}$. We shall henceforth refer to $m$ as the order of the amplitude. Following the folklore in harmonic and microlocal analysis, we shall refer to the class $S_{0, \delta}^{m}\left(\mathbb{R}^{n}\right)$ as the exotic class and to $S_{\rho, 1}^{m}\left(\mathbb{R}^{n}\right)$ as the forbidden class of amplitudes.

Towards the end of this paper, in connection with the amplitudes with low spatial regularity and also the forbidden amplitudes, we will use the Zygmund class $C_{*}^{r}\left(\mathbb{R}^{n}\right)$ whose definition we now recall.

Definition 2.9 Let $r \in \mathbb{R}$. The Zygmund class is defined by

$$
C_{*}^{r}\left(\mathbb{R}^{n}\right):=\left\{f \in \mathscr{S}^{\prime}\left(\mathbb{R}^{n}\right):\|f\|_{C_{*}^{r}\left(\mathbb{R}^{n}\right)}:=\sup _{j \geq 0} 2^{j r}\left\|\psi_{j}(D) f\right\|_{L^{\infty}\left(\mathbb{R}^{n}\right)}<\infty\right\} .
$$

If $C^{r}\left(\mathbb{R}^{n}\right), r \in \mathbb{R}_{+}$, denotes the Hölder space, and $\mathcal{C}^{r}\left(\mathbb{R}^{n}\right)$ denotes the space of continuous functions with continuous derivatives of orders up to and including $r$, then one also has that

$$
C_{*}^{r}\left(\mathbb{R}^{n}\right)=C^{r}\left(\mathbb{R}^{n}\right) \text { for } r \in \mathbb{R}_{+} \backslash \mathbb{Z}_{+} \text {and } \mathcal{C}^{r}\left(\mathbb{R}^{n}\right) \subset C_{*}^{r}\left(\mathbb{R}^{n}\right) \text { for } r \in \mathbb{Z}_{+} \text {. }
$$

In connection to the definition of the Zygmund class, there is another class of amplitudes which have low regularity in the $x$-variable, which were considered by $\mathrm{G}$. Bourdaud in [3].

Definition 2.10 Let $m \in \mathbb{R}, 0 \leq \delta \leq 1$ and $r>0$. An amplitude (symbol) $a(x, \xi)$ is in the class $C_{*}^{r} S_{1, \delta}^{m}\left(\mathbb{R}^{n}\right)$ if it is $\overline{\mathcal{C}}^{\infty}\left(\mathbb{R}^{n}\right)$ in the $\xi$ variable and verifies the estimates

$$
\left\|\partial_{\xi}^{\alpha} a(\cdot, \xi)\right\|_{L^{\infty}\left(\mathbb{R}^{n}\right)} \lesssim\langle\xi\rangle^{m-|\alpha|}
$$

and

$$
\left\|\partial_{\xi}^{\alpha} a(\cdot, \xi)\right\|_{C_{*}^{r}\left(\mathbb{R}^{n}\right)} \lesssim\langle\xi\rangle^{m-|\alpha|+\delta r},
$$

for all multi-indices $\alpha$ and $\xi \in \mathbb{R}^{n}$. Here $C_{*}^{r}\left(\mathbb{R}^{n}\right)$ is the Zygmund class of Definition 2.9 .

It is important to note that $S_{1,1}^{m}\left(\mathbb{R}^{n}\right) \subset C_{*}^{r} S_{1,1}^{m}\left(\mathbb{R}^{n}\right)$, for all $r>0$, which follows from (3).

Given the symbol classes defined above, one associates to the symbol its KohnNirenberg quantisation as follows: 
Definition 2.11 Let $a$ be a symbol. Define a pseudodifferential operator ( $\Psi$ DO for short) as the operator

$$
a(x, D) f(x):=\int_{\mathbb{R}^{n}} e^{i x \cdot \xi} a(x, \xi) \widehat{f}(\xi) \mathrm{d} \xi,
$$

a priori defined on the Schwartz class $\mathscr{S}\left(\mathbb{R}^{n}\right)$.

In order the define the Fourier integral operators that are studied in this paper, following [11], we also define the classes of phase functions.

Definition 2.12 A phase function $\varphi(x, \xi)$ in the class $\Phi^{k}$ is a function $\varphi(x, \xi) \in \mathcal{C}^{\infty}\left(\mathbb{R}^{n} \times \mathbb{R}^{n} \backslash\{0\}\right)$, positively homogeneous of degree one in the frequency variable $\xi$ satisfying the following estimate

$$
\sup _{(x, \xi) \in \mathbb{R}^{n} \times \mathbb{R}^{n} \backslash\{0\}}|\xi|^{-1+|\alpha|}\left|\partial_{\xi}^{\alpha} \partial_{x}^{\beta} \varphi(x, \xi)\right| \leq C_{\alpha, \beta},
$$

for any pair of multi-indices $\alpha$ and $\beta$, satisfying $|\alpha|+|\beta| \geq k$. In this paper we will mainly use phases in class $\Phi^{2}$ and $\Phi^{1}$.

We will also need to consider phase functions that satisfy certain non-degeneracy conditions. These conditions have to be adapted to the case of local and global boundedness in an appropriate way. Following [23], in connection to the investigation of the local results, that is, under the assumption that the $x$-support of the amplitude $a(x, \xi)$ lies within a fixed compact set $\mathcal{K}$, the non-degeneracy condition is formulated as follows:

Definition 2.13 Let $\mathcal{K}$ be a fixed compact subset of $\mathbb{R}^{n}$. One says that the phase function $\varphi(x, \xi)$ satisfies the non-degeneracy condition if

$$
\operatorname{det}\left(\partial_{x_{j} \xi_{k}}^{2} \varphi(x, \xi)\right) \neq 0, \quad \text { for all }(x, \xi) \in \mathcal{K} \times \mathbb{R}^{n} \backslash\{0\}
$$

Following the approaches in e.g. $[11,21,22]$, for the global $L^{p}$-boundedness results that were established in those papers, we also define the following somewhat stronger notion of non-degeneracy:

Definition 2.14 One says that the phase function $\varphi(x, \xi)$ satisfies the strong nondegeneracy condition (or $\varphi$ is SND for short) if

$$
\left|\operatorname{det}\left(\partial_{x_{j} \xi_{k}}^{2} \varphi(x, \xi)\right)\right| \geq \delta, \quad \text { for some } \delta>0 \text { and all }(x, \xi) \in \mathbb{R}^{n} \times \mathbb{R}^{n} \backslash\{0\} \text {. }
$$

Having the definitions of the amplitudes and the phase functions at hand, one has

Definition 2.15 A Fourier integral operator (FIO for short) $T_{a}^{\varphi}$ with amplitude $a$ and phase function $\varphi$, is an operator defined (once again a-priori on $\mathscr{S}\left(\mathbb{R}^{n}\right)$ ) by

$$
T_{a}^{\varphi} f(x):=\int_{\mathbb{R}^{n}} e^{i \varphi(x, \xi)} a(x, \xi) \widehat{f}(\xi) \mathrm{d} \xi,
$$


where $\varphi(x, \xi) \in \mathcal{C}^{\infty}\left(\mathbb{R}^{n} \times \mathbb{R}^{n} \backslash\{0\}\right)$ and is positively homogeneous of degree one in $\xi$.

In this paper, the basic $L^{2}$-boundedness result which we shall utilise for the FIOs, is the following proposition which could be found in [11] as Theorems 2.2 and 2.7.

Proposition 2.16 Let $\rho, \delta \in[0,1], \delta \neq 1$. Assume that $a(x, \xi) \in S_{\rho, \delta}^{m}\left(\mathbb{R}^{n}\right)$ and $\varphi(x, \xi)$ is in the class $\Phi^{2}$ and is SND. Then the FIO $T_{a}^{\varphi}$ is bounded on $L^{2}\left(\mathbb{R}^{n}\right)$ if and only if $m=-n \max (0,(\delta-\rho) / 2)$. In case $\rho \in[0,1], \delta=1$ then the $L^{2}$-boundedness is valid if and only if $m<n(\rho-1) / 2$.

A global result concerning the boundedness of FIOs with amplitudes of order zero, which will be used in the proof of Proposition 7.2 goes as follows:

Lemma 2.17 Let $a(x, \xi) \in S_{1,0}^{0}\left(\mathbb{R}^{n}\right)$. Assume also that $\varphi(x, \xi) \in \Phi^{2}$, is SND. Then for $s \in \mathbb{R}$, the FIO $T_{a}^{\varphi}$ is bounded from the Sobolev space $H^{s}\left(\mathbb{R}^{n}\right)$ to $H^{s}\left(\mathbb{R}^{n}\right)$.

Proof This follows immediately from [16, Theorem 5.7 part (ii)], by noting that the Besov-Lipschitz space $B_{p, q}^{s}\left(\mathbb{R}^{n}\right)$ in that result reduces to the Sobolev space $H^{s}\left(\mathbb{R}^{n}\right)$ when $p=q=2$.

We also state the following version of the non-stationary phase lemma, whose proof can be found in [21, Lemma 3.2].

Lemma 2.18 Let $\mathcal{K} \subset \mathbb{R}^{n}$ be a compact set and $\Omega \supset \mathcal{K}$ an open set. Assume that $\Phi$ is a real valued function in $\mathcal{C}^{\infty}(\Omega)$ such that $|\nabla \Phi|>0$ and

$$
\left|\partial^{\alpha} \Phi\right| \lesssim|\nabla \Phi|
$$

for all multi-indices $\alpha$ with $|\alpha| \geq 1$. Then, for any $F \in \mathcal{C}_{c}^{\infty}(\mathcal{K}), \lambda>0$, and any integer $k \geq 0$,

$$
\left|\int_{\mathbb{R}^{n}} F(\xi) e^{i \lambda \Phi(\xi)} \mathrm{d} \xi\right| \leq C_{k, n, \mathcal{K}} \lambda^{-k} \sum_{|\alpha| \leq k} \int_{\mathcal{K}}\left|\partial^{\alpha} F(\xi)\right||\nabla \Phi(\xi)|^{-k} \mathrm{~d} \xi
$$

Finally we recall a composition result, whose proof can be found in [20, Theorem 4.2], or in a more general setting in [4, Theorem 3.11], which will enable us to keep track of the parameter while a parameter-dependent $\Psi$ DO acts from the left on a parameterdependent FIO. This will be crucial in the proof of the boundedness of FIOs with forbidden amplitudes on Sobolev spaces.

Proposition 2.19 Let $m \leq 0,0<\varepsilon<1 / 2$ and $\Omega:=\mathbb{R}^{n} \times\{|\xi|>1\}$. Suppose that $a_{t}(x, \xi) \in S_{1,0}^{m}\left(\mathbb{R}^{n}\right)$ uniformly in $t \in(0,1]$ and it is supported in $\Omega, b(\xi) \in S_{1,0}^{0}\left(\mathbb{R}^{n}\right)$ and $\varphi \in \mathcal{C}^{\infty}(\Omega)$ is such that

(i) for constants $C_{1}, C_{2}>0, C_{1}|\xi| \leq\left|\nabla_{x} \varphi(x, \xi)\right| \leq C_{2}|\xi|$ for all $(x, \xi) \in \Omega$, and

(ii) for all $|\alpha|,|\beta| \geq 1,\left|\partial_{x}^{\alpha} \varphi(x, \xi)\right| \lesssim\langle\xi\rangle$ and $\left|\partial_{\xi}^{\alpha} \partial_{x}^{\beta} \varphi(x, \xi)\right| \lesssim|\xi|^{1-|\alpha|}$, for all $(x, \xi) \in \Omega$. 
Consider the parameter dependent Fourier integral operator $T_{a_{t}}^{\varphi}$, given by (6) with amplitude $a_{t}(x, \xi)$, and the parameter dependent Fourier multiplier

$$
b(t D) f(x):=\int_{\mathbb{R}^{n}} e^{i x \cdot \xi} b(t \xi) \widehat{f}(\xi) đ \xi .
$$

Then the composition $b(t D) T_{a_{t}}^{\varphi}$ is also an FIO with phase $\varphi$ and amplitude $\sigma_{t}$ which is given by

$$
\sigma_{t}(x, \xi):=\iint_{\mathbb{R}^{n} \times \mathbb{R}^{n}} a_{t}(y, \xi) b(t \eta) e^{i(x-y) \cdot \eta+i \varphi(y, \xi)-i \varphi(x, \xi)} \mathrm{d} \eta \mathrm{d} y .
$$

Moreover, for each $M \geq 1$, we can write $\sigma_{t}$ as

$$
\sigma_{t}(x, \xi)=b\left(t \nabla_{x} \varphi(x, \xi)\right) a_{t}(x, \xi)+\sum_{0<|\alpha|<M} \frac{t^{|\alpha|}}{\alpha !} \sigma_{\alpha}(t, x, \xi)+t^{M \varepsilon} r(t, x, \xi),
$$

for $t \in(0,1)$. Moreover, for all multi-indices $\beta, \gamma$ one has

$$
\sup _{t \in(0,1)}\left|\partial_{\xi}^{\gamma} \partial_{x}^{\beta} \sigma_{\alpha}(t, x, \xi) t^{|\alpha|(1-\varepsilon)}\right| \lesssim\langle\xi\rangle^{m-|\alpha|(1 / 2-\varepsilon)-|\gamma|} \text { for } 0<|\alpha|<M
$$

and

$$
\sup _{t \in(0,1)}\left|\partial_{\xi}^{\gamma} \partial_{x}^{\beta} r(t, x, \xi)\right| \lesssim\langle\xi\rangle^{m-M(1 / 2-\varepsilon)-|\gamma|}
$$

\section{Reduction}

We start by describing how the problem of $L^{p}$-boundedness of FIOs with SND phase functions that belong to the class $\Phi^{2}$, can be reduced to the case of operators that are well-suited for the Ruzhansky-Sugimoto's globalisation procedure.

Thus let $T_{a}^{\varphi}$ be an FIO given by (6), with $\varphi \in \Phi^{2}$ and SND, and an amplitude $a(x, \xi) \in S_{\rho, \delta}^{m}\left(\mathbb{R}^{n}\right)$ with $m \in \mathbb{R}, \rho, \delta \in[0,1]$. We start by localising the amplitude in the $\xi$ variable by introducing an open convex covering $\left\{U_{l}\right\}_{l=1}^{M}$ of the unit sphere $\mathbb{S}^{n-1}$, where $M$ is finite (due to compactness of $\mathbb{S}^{n-1}$ ) but large enough, and the sets $U_{l}$ have diameters of at most $d$. Let $\Xi_{l}$ be a smooth partition of unity subordinate to the covering $U_{l}$ and set

$$
a_{l}(x, \xi):=a(x, \xi) \Xi_{l}\left(\frac{\xi}{|\xi|}\right)
$$

Define

$$
T_{l} f(x):=\int_{\mathbb{R}^{n}} a_{l}(x, \xi) e^{i \varphi(x, \xi)} \widehat{f}(\xi) đ \xi
$$


and fix a point $\zeta_{l} \in U_{l}$. Then for any $\xi \in U_{l}$, Taylor's formula and Euler's homogeneity formula yield

$$
\begin{aligned}
\varphi(x, \xi) & =\varphi\left(x, \zeta_{l}\right)+\nabla_{\xi} \varphi\left(x, \zeta_{l}\right) \cdot\left(\xi-\zeta_{l}\right)+\lambda(x, \xi) \\
& =\lambda(x, \xi)+\nabla_{\xi} \varphi\left(x, \zeta_{l}\right) \cdot \xi
\end{aligned}
$$

where $\lambda$ is the remainder term and its dependence on $\zeta_{l}$ has been suppressed due the fact that $\zeta_{l}$ was fixed. Thus for $\xi \in U_{l}$, using the fact that $\partial_{\xi_{k}} \varphi(x, \xi)$ is homogeneous of degree zero in $\xi$, we have

$$
\partial_{\xi_{k}} \lambda(x, \xi)=\partial_{\xi_{k}} \varphi\left(x, \frac{\xi}{|\xi|}\right)-\partial_{\xi_{k}} \varphi\left(x, \zeta_{l}\right),
$$

and hence the mean-value theorem and the definition of class $\Phi^{2}$ yield

$$
\left|\partial_{\xi}^{\alpha} \partial_{x}^{\beta} \lambda(x, \xi)\right| \leq C|\xi|^{1-|\alpha|},
$$

for all $|\beta| \geq 0$, and for $|\alpha| \geq 2$. One also observes that due to the homogeneity of $\lambda(x, \xi)$ in $\xi$ and the mean-value theorem, one also has that $\left|\nabla_{x} \lambda(x, \xi)\right| \lesssim|\xi|$. We shall now extend the function $\lambda(x, \xi)$ to the whole of $\mathbb{R}^{n} \times \mathbb{R}^{n} \backslash\{0\}$, preserving its properties and we denote this extension by $\lambda(x, \xi)$ again. Now this $\lambda$ belongs to the class $\Phi^{1}$. Hence the Fourier integral operators $T_{l}$ defined by

$$
T_{l} f(x):=\int_{\mathbb{R}^{n}} a_{l}(x, \xi) e^{i \lambda(x, \xi)+i \nabla_{\xi} \varphi\left(x, \zeta_{l}\right) \cdot \xi} \widehat{f}(\xi) \mathrm{d} \xi,
$$

are the localised pieces of the original Fourier integral operator $T$ and therefore

$$
T=\sum_{l=1}^{M} T_{l}
$$

Now, let us investigate the $L^{p}$-boundedness of each piece $T_{l}$. To this end we observe that due to the SND assumption on $\varphi$, the map $\mathbf{t}_{l}(x):=\nabla_{\xi} \varphi\left(x, \zeta_{l}\right)$ is a global diffeomorphism and composing $T_{l} f(x)$ with the inverse of $\mathbf{t}_{l}$ results in the FIO

$$
\left(T_{l} f\right)\left(\mathbf{t}_{l}^{-1}(x)\right)=\int_{\mathbb{R}^{n}} a_{l}\left(\mathbf{t}_{l}^{-1}(x), \xi\right) e^{i \lambda\left(\mathbf{t}_{l}^{-1}(x), \xi\right)+i x \cdot \xi} \widehat{f}(\xi) \mathrm{d} \xi
$$

Observe that all the derivatives of $\mathbf{t}_{l}^{-1}$ are bounded and indeed the phase function $\lambda\left(\mathbf{t}_{l}^{-1}(x), \xi\right)+x \cdot \xi$ is SND (note also that the diameters $d$ can be picked as small as we like). Therefore the study of the global $L^{p}$-boundedness of $T_{l}$ is reduced to the study of the global $L^{p}$-boundedness of FIOs of the form

$$
\int_{\mathbb{R}^{n}} \sigma(x, \xi) e^{i \theta(x, \xi)+i x \cdot \xi} \widehat{f}(\xi) \mathrm{d} \xi
$$


where $\sigma(x, \xi)$ belongs to the same amplitude-class as $a(x, \xi)$ (i.e. $\left.S_{\rho, \delta}^{m}\left(\mathbb{R}^{n}\right)\right), \theta \in \Phi^{1}$ and $\theta(x, \xi)+x \cdot \xi$ being SND.

In a similar way, one can show that, for an FIO of the form

$$
\iint_{\mathbb{R}^{n} \times \mathbb{R}^{n}} a(y, \xi) e^{i \varphi(y, \xi)-i x \cdot \xi} f(y) \mathrm{d} \xi \mathrm{d} y,
$$

with $\varphi \in \Phi^{2}$, matters can be reduced to FIOs of the form

$$
\iint_{\mathbb{R}^{n} \times \mathbb{R}^{n}} \sigma(y, \xi) e^{i \theta(y, \xi)+i(y-x) \cdot \xi} f(y) \mathrm{d} \xi \mathrm{d} y,
$$

where $\sigma(y, \xi)$ belongs to the same class as $a(y, \xi)$ and $\theta(y, \xi) \in \Phi^{1}$.

\section{Composition of Fourier multipliers and FIOs}

We start with a composition theorem which allows us to left-compose a Fourier multiplier with an FIO. The difference between Theorem 4.1 below and Proposition 2.19 lies in the fact that, although the latter deals with the parameter dependent case, it only covers amplitudes in $S_{1,0}^{m}\left(\mathbb{R}^{n}\right)$. Also the method of proof of Proposition 2.19 is quite different from that of the following theorem whose proof is not just a modification of the former. The difficulties arise exactly when $\delta \geq \rho$, but they can be overcome. However, as we shall see, the forbidden case of $\delta=1$ has to be excluded.

Note that the composition theorem below also allows us to compose our more general FIOs with Bessel potential operators (see i.e. Lemma 6.3) in order to obtain crucial $\mathscr{H}^{q}-L^{2}$ estimates that are in turn used in the proofs of the $L^{p}$-boundedness results (Proposition 6.4).

Theorem 4.1 Let $m, m^{\prime} \in \mathbb{R}, \rho \in[0,1], \delta \in[0,1)$ and $\Omega:=\mathbb{R}^{n} \times\{|\xi|>1\}$. Suppose that $a(x, \xi) \in S_{\rho, \delta}^{m}\left(\mathbb{R}^{n}\right)$, and it is supported in $\Omega, \gamma(\xi) \in S_{1,0}^{m^{\prime}}\left(\mathbb{R}^{n}\right)$ and $\varphi \in \mathcal{C}^{\infty}(\Omega)$ is such that

(i) for constants $C_{1}, C_{2}>0, C_{1}|\xi| \leq\left|\nabla_{x} \varphi(x, \xi)\right| \leq C_{2}|\xi|$ for all $(x, \xi) \in \Omega$, and

(ii) for all $|\alpha|,|\beta| \geq 1,\left|\partial_{x}^{\alpha} \varphi(x, \xi)\right| \lesssim\langle\xi\rangle$ and $\left|\partial_{\xi}^{\alpha} \partial_{x}^{\beta} \varphi(x, \xi)\right| \lesssim 1$, for all $(x, \xi) \in \Omega$.

Consider the Fourier multiplier and the Fourier integral operator

$\gamma(D) f(x):=\int_{\mathbb{R}^{n}} e^{i x \cdot \xi} \gamma(\xi) \widehat{f}(\xi) \mathrm{d} \xi$ and $T_{a}^{\varphi} f(x):=\int_{\mathbb{R}^{n}} e^{i \varphi(x, \xi)} a(x, \xi) \widehat{f}(\xi) \mathrm{d} \xi$.

Then the composition operator $T_{b}^{\varphi}:=\gamma(D) T_{a}^{\varphi}$ is also an FIO (with the same phase as $T_{a}^{\varphi}$ ), and with amplitude given by

$$
b(x, \xi):=\iint_{\mathbb{R}^{n} \times \mathbb{R}^{n}} a(y, \xi) \gamma(\eta) e^{i(x-y) \cdot \eta+i \varphi(y, \xi)-i \varphi(x, \xi)} \mathrm{d} \eta \mathrm{d} y .
$$

Moreover $b \in S_{\rho, \delta}^{m+m^{\prime}}\left(\mathbb{R}^{n}\right)$. 
Remark 4.2 It is easy to show that if a phase function $\varphi \in \Phi^{2}$ is $S N D$ then it satisfies all the requirements of Theorem 4.1 .

Proof of Theorem 4.1 The expression in (7) can easily be derived through a simple calculation. Set

$$
c(x, \xi, \eta):=a(x, \xi) \gamma(\eta)
$$

and

$$
\Phi(x, y, \xi, \eta):=(x-y) \cdot \eta+\varphi(y, \xi)-\varphi(x, \xi)
$$

Then the following estimates are valid:

$$
\begin{aligned}
\left\langle\nabla_{\eta} \Phi\right\rangle & =\langle x-y\rangle, \\
\left\langle\nabla_{y} \Phi\right\rangle & \geq C_{R}\langle\xi-\eta\rangle, \quad|\xi| \leq R, \quad R>0, \\
\left|\nabla_{x} \Phi\right| & \lesssim|\xi|+|\xi-\eta|, \\
\left|\nabla_{\xi} \Phi\right| & \lesssim|x-y| .
\end{aligned}
$$

Indeed the first equality is trivial, and for the second one, setting

$$
\Lambda(x, \xi):=\varphi(x, \xi)-x \cdot \xi
$$

we note that for $|\xi|<R$ and $R \geq 1$, triangle inequality and condition $(i)$ on the phase yield that

$$
\begin{aligned}
2\left(C_{2}+1\right) R\left(1+\left|\nabla_{y} \Phi\right|\right) & \geq 2\left(C_{2}+1\right) R+\left|\nabla_{y} \Phi\right| \\
& =2\left(C_{2}+1\right) R+\left|\nabla_{y}(\varphi(y, \xi)-y \cdot \eta)\right| \\
& =2\left(C_{2}+1\right) R+\left|\nabla_{y}(y \cdot(\xi-\eta)+\Lambda(y, \xi))\right| \\
& =2\left(C_{2}+1\right) R+\left|\xi-\eta+\nabla_{y} \Lambda(y, \xi)\right| \\
& \geq 2\left(C_{2}+1\right) R+|\xi-\eta|-\left(C_{2}+1\right)|\xi| \\
& \geq 2\left(C_{2}+1\right) R-\left(C_{2}+1\right) R+|\xi-\eta| \\
& =\left(C_{2}+1\right) R+|\xi-\eta| \\
& \geq(1+|\xi-\eta|) .
\end{aligned}
$$

On the other hand, for $|\xi|<R$ and $R<1$, condition $(i)$ on the phase implies

$$
\begin{aligned}
\left(C_{2}+1\right)\left(1+\left|\nabla_{y} \Phi\right|\right) & \geq\left(C_{2}+1\right)+\frac{1}{2}\left|\nabla_{y} \Phi\right| \\
& =\left(C_{2}+1\right)+\frac{1}{2}\left|\xi-\eta+\nabla_{y} \Lambda(y, \xi)\right| \\
& \geq\left(C_{2}+1\right)+\frac{1}{2}|\xi-\eta|-\frac{C_{2}+1}{2}|\xi|
\end{aligned}
$$




$$
\begin{aligned}
& \geq\left(C_{2}+1\right)-\frac{R\left(C_{2}+1\right)}{2}+\frac{1}{2}|\xi-\eta| \\
& \geq \frac{1}{2}(1+|\xi-\eta|) .
\end{aligned}
$$

To show the third estimate in (8) we observe that condition $(i)$ on the phase yields

$$
\left|\nabla_{x} \Phi\right|=\left|\nabla_{x}(x \cdot(\eta-\xi)-\Lambda(x, \xi))\right| \lesssim|\xi-\eta|+\left(C_{2}+1\right)|\xi| \lesssim|\xi-\eta|+|\xi|
$$

Finally to show the fourth estimate in (8) we observe that the mean-value theorem and condition $(i i)$ on the phase yield that

$$
\left|\nabla_{\xi} \Phi\right|=\left|\nabla_{\xi} \varphi(y, \xi)-\nabla_{\xi} \varphi(x, \xi)\right| \lesssim|x-y| .
$$

Using Faà di Bruno's formulae and estimate (8) we can also show that

$$
\left|\partial_{x}^{\beta} \partial_{\xi}^{\alpha} e^{i \Phi}\right| \lesssim\langle x-y\rangle^{|\alpha|}\left(1+|\xi|^{2}+|\xi-\eta|^{2}\right)^{|\beta| / 2} .
$$

Introduce the differential operators

$$
\begin{aligned}
& L_{\eta}:=\left\langle\nabla_{\eta} \Phi\right\rangle^{-2}\left(1-i \nabla_{\eta} \Phi \cdot \nabla_{\eta}\right) \\
& L_{y}:=\left\langle\nabla_{y} \Phi\right\rangle^{-2}\left(1-i \nabla_{y} \Phi \cdot \nabla_{y}\right),
\end{aligned}
$$

and integrating by parts we have

$$
b(x, \xi)=\iint_{\mathbb{R}^{n} \times \mathbb{R}^{n}} e^{i \Phi}\left(L_{\eta}^{*}\right)^{M}\left(L_{y}^{*}\right)^{N} c(y, \xi, \eta) \mathrm{d} \eta \mathrm{d} y,
$$

for large positive $N$ and $M$. Note that

$$
\left|\partial_{\xi}^{\alpha} \partial_{y}^{\beta} \partial_{\eta}^{\gamma} c(y, \xi, \eta)\right| \lesssim\langle\xi\rangle^{m-\rho|\alpha|+\delta|\beta|}\langle\eta\rangle^{m^{\prime}-|\gamma|},
$$

for all multi-indices $\alpha, \beta, \gamma$. It follow from this and (8) that $b(x, \xi)$ is bounded on $\mathbb{R}_{x}^{n} \times B(0, R)$ for $R>0$, and using (9) one can also show that, for any multiindices $\alpha, \beta, \partial_{\xi}^{\alpha} \partial_{x}^{\beta} b(x, \xi)$ is also bounded on $\mathbb{R}_{x}^{n} \times B(0, R)$ and therefore $b(x, \xi) \in$ $\mathcal{C}_{b}^{\infty}\left(\mathbb{R}_{x}^{n} \times B(0, R)\right)$ (the subscript $b$ indicates the boundedness of all the derivatives). It also follows from condition $(i)$ on the phase that

$$
\left|\nabla_{y} \Phi\right|=\left|\nabla_{y} \varphi(y, \xi)-\eta\right| \geq C_{1}|\xi|-|\eta|
$$

Now let $\chi \in \mathcal{C}_{c}^{\infty}\left(\mathbb{R}^{n}\right)$ be such that $0 \leq \chi(x) \leq 1$ and $\chi(x)=1$ when $|x| \leq 1 / 2$ and $\chi(x)=0$ when $|x| \geq 2 / 3$. Set

$$
\chi_{1}(\eta, \xi):=\chi\left(\frac{\eta}{C_{1}\langle\xi\rangle}\right) .
$$


Since $|\eta| \leq 2 C_{1}\langle\xi\rangle / 3$ on the support of $\chi_{1},(11)$ yields (on supp $\chi_{1}$ )

$$
\left|\nabla_{y} \Phi\right| \geq C_{1}\left(|\xi|-\frac{2}{3}\langle\xi\rangle\right)
$$

At this point, we observe that since

$$
\lim _{|\xi| \rightarrow \infty} C_{1}\left(\frac{|\xi|}{\langle\xi\rangle}-\frac{2}{3}\right)=\frac{C_{1}}{3}
$$

it follows that there exists $R_{1}>0$ and $C>0$ such that on supp $\chi_{1} \cap\left\{|\xi| \geq R_{1}\right\}$ one has

$$
\left|\nabla_{y} \Phi\right| \geq C\langle\xi\rangle=C\left(\frac{1}{2}+\frac{|\xi|^{2}}{2}+\frac{\langle\xi\rangle^{2}}{2}\right)^{1 / 2} \gtrsim\left(1+|\xi|^{2}+|\eta|^{2}\right)^{1 / 2} .
$$

Setting

$$
\begin{gathered}
b_{1}(x, \xi):=\iint_{\mathbb{R}^{n} \times \mathbb{R}^{n}} c(y, \xi, \eta) \chi_{1}(\eta, \xi) e^{i \Phi} \mathrm{d} \eta \mathrm{d} y, \\
\tilde{L}_{y}:=-i\left|\nabla_{y} \Phi\right|^{-2} \nabla_{y} \Phi \cdot \nabla_{y}
\end{gathered}
$$

and integrating by parts yields

$$
b_{1}(x, \xi)=\iint_{\mathbb{R}^{n} \times \mathbb{R}^{n}} e^{i \Phi}\left(L_{\eta}^{*}\right)^{M}\left(\tilde{L}_{y}^{*}\right)^{N}\left(\chi_{1}(\eta, \xi) c(y, \xi, \eta)\right) đ \eta \mathrm{d} y .
$$

Since $b(x, \xi) \in \mathcal{C}_{b}^{\infty}\left(\mathbb{R}_{x}^{n} \times B(0, R)\right)$, estimates (9) and (12) yield that $b_{1}(x, \xi) \in$ $S^{-\infty}\left(\mathbb{R}^{n}\right)$ (first we show using (10) that $\left|b_{2}(x, \xi)\right| \lesssim\langle\xi\rangle^{-N}$, for all $N \geq 0$, then using (9) we boost up this result to all the derivatives of $b(x, \xi)$, for more details on these type of calculations, see e.g. the somewhat similar proof of Theorem 3.11 in [4]).

Now define $\chi_{2}:=1-\chi_{1}$ and consider

$$
b_{2}(x, \xi):=\iint_{\mathbb{R}^{n} \times \mathbb{R}^{n}} c(y, \xi, \eta) \chi_{2}(\eta, \xi) e^{i \Phi} \mathrm{d} \eta \mathrm{d} y .
$$

To simplify the calculations from here we set

$$
I(x, y, \xi):=\int_{0}^{1} \nabla_{x} \Lambda(y+s(x-y), \xi) \mathrm{d} s
$$

Noting that $\nabla_{x} \varphi(x, \xi)=\nabla_{x} \Lambda(x, \xi)+\xi$, rewriting

$$
\Phi(x, y, \xi, \eta)=(x-y) \cdot(\eta-\xi-I(x, y, \xi))
$$

making the change of variables

$$
z:=y-x, \quad \zeta:=\eta-\xi-I(x, y, \xi)
$$


and then defining

$$
c_{1}(y, \xi, \eta):=\chi_{2}(\eta+I(x, y, \xi), \xi) c(y, \xi, \eta+I(x, y, \xi))
$$

we obtain

$$
b_{2}(x, \xi)=\iint_{\mathbb{R}^{n} \times \mathbb{R}^{n}} e^{-i z \cdot \zeta} c_{1}(x+z, \xi, \xi+\zeta) \mathrm{d} \zeta \mathrm{d} z .
$$

Moreover, $b_{2}(x, \xi)$ in turn can be split into $b_{3}(x, \xi)+b_{4}(x, \xi)$ with

$$
b_{3}(x, \xi):=\iint_{\mathbb{R}^{n} \times \mathbb{R}^{n}} e^{-i z \cdot \zeta} \chi_{2}(\zeta, \xi) c_{1}(x+z, \xi, \xi+\zeta) \mathrm{d} \zeta \mathrm{d} z
$$

and

$$
b_{4}(x, \xi):=\iint_{\mathbb{R}^{n} \times \mathbb{R}^{n}} e^{-i z \cdot \zeta} \chi_{1}(\zeta, \xi) c_{1}(x+z, \xi, \xi+\zeta) \mathrm{d} \zeta \mathrm{d} z .
$$

We observe that on the support of $\chi_{2}(\zeta, \xi)$ one has that

$$
|\zeta| \geq \frac{1}{2} C_{1}\langle\xi\rangle
$$

and on the support of $\chi_{2}(I(x, y, \xi)+\xi+\zeta, \xi)$,

$$
|I(x, y, \xi)+\xi+\zeta| \geq \frac{1}{2} C_{1}\langle\xi\rangle .
$$

Therefore integrating by parts we can show that

$$
\begin{aligned}
b_{3}(x, \xi)= & \iint_{\mathbb{R}^{n} \times \mathbb{R}^{n}} e^{-i z \cdot \zeta}|\zeta|^{-2 N} \\
& \times\left(-\Delta_{z}\right)^{N}\left\{\langle z\rangle^{-2 N}\left(1-\Delta_{\zeta}\right)^{N}\left(\chi_{2}(\zeta, \xi) c_{1}(x+z, \xi, \xi+\zeta)\right)\right\} \mathrm{d} \zeta \mathrm{d} z
\end{aligned}
$$

to conclude that $b_{3}(x, \xi) \in S^{-\infty}\left(\mathbb{R}^{n}\right)$.

Defining

$$
c_{2}(y, \xi, \eta):=\chi_{1}(\eta-\xi, \xi) c_{1}(y, \xi, \eta)
$$

we see that

$$
c_{2}(y, \xi, \eta)=\chi_{1}(\eta-\xi, \xi) \chi_{2}(\eta+I(x, y, \xi), \xi) c(y, \xi, \eta+I(x, y, \xi))
$$

and $b_{4}(x, \xi)$ can be written as

$$
b_{4}(x, \xi)=\iint_{\mathbb{R}^{n} \times \mathbb{R}^{n}} e^{-i z \cdot \zeta} c_{2}(x+z, \xi, \xi+\zeta) đ \zeta \mathrm{d} z
$$


Using the definition of $c_{2}(y, \xi, \eta)$ we see that

$$
\begin{aligned}
& |\eta-\xi| \leq \frac{2}{3} C_{1}\langle\xi\rangle \\
& |\eta+I(x, y, \xi)| \geq \frac{1}{2} C_{1}\langle\xi\rangle,
\end{aligned}
$$

on the support of $c_{2}(y, \xi, \eta)$.

In what follows we shall denote the derivative of $c_{2}(y, \xi, \eta)$ with respect to $y$ by $\partial_{1} c_{2}$, the derivative of $c_{2}(y, \xi, \eta)$ w.r.t. $\xi$ by $\partial_{2} c_{2}$, and the derivative of $c_{2}(y, \xi, \eta)$ w.r.t. $\eta$ by $\partial_{3} c_{2}$. Now using (13) and Taylor's formula we have that

$$
\begin{aligned}
& b_{4}(x, \xi)=\sum_{|\nu|<N} \frac{(-i)^{|v|}}{\nu !} \iint_{\mathbb{R}^{n} \times \mathbb{R}^{n}} e^{-i z \cdot \zeta}\left(\partial_{1}^{v} \partial_{3}^{v} c_{2}\right)(x+z, \xi, \xi) \mathrm{d} \zeta \mathrm{d} z \\
& \quad+N \sum_{|\nu|=N} \int_{0}^{1} \frac{(1-s)^{N-1}}{\nu !} \iint_{\mathbb{R}^{n} \times \mathbb{R}^{n}} e^{-i z \cdot \zeta}\left(\partial_{1}^{v} \partial_{3}^{v} c_{2}\right)(x+z, \xi, \xi+s \zeta) \mathrm{d} \zeta \mathrm{d} z \mathrm{~d} s .
\end{aligned}
$$

For $s \in[0,1]$, set

$$
\sigma(x+z, \xi, \xi+s \zeta):=\left(\partial_{1}^{v} \partial_{3}^{v} c_{2}\right)(x+z, \xi, \xi+s \zeta)
$$

Let us now study the behaviour of the derivatives of

$$
r_{s}(x, \xi):=\iint_{\mathbb{R}^{n} \times \mathbb{R}^{n}} e^{-i z \cdot \zeta} \sigma(x+z, \xi, \xi+s \zeta) \mathrm{d} \zeta \mathrm{d} z .
$$

To this end observe that

$$
\partial_{\xi}^{\alpha} \partial_{x}^{\beta} r_{s}(x, \xi)=\iint_{\mathbb{R}^{n} \times \mathbb{R}^{n}} e^{-i z \cdot \zeta} \partial_{\xi}^{\alpha} \partial_{x}^{\beta} \sigma(x+z, \xi, \xi+s \zeta) \mathrm{d} \zeta \mathrm{d} z .
$$

Now for $M>n / 2$ we write

$$
e^{i z \cdot \zeta}=\left(1+\langle\xi\rangle^{2 \delta}|z|^{2}\right)^{-M}\left(1+\langle\xi\rangle^{2 \delta}\left(-\Delta_{\zeta}\right)\right)^{M} e^{i z \cdot \zeta}
$$

and integration by parts yields that

$$
\partial_{\xi}^{\alpha} \partial_{x}^{\beta} r_{s}(x, \xi)=\iint_{\mathbb{R}^{n} \times \mathbb{R}^{n}} e^{-i z \cdot \zeta} R_{s}(x, \xi, z, \zeta) \mathrm{d} \zeta \mathrm{d} z,
$$

with

$$
R_{S}(x, \xi, z, \zeta):=\left(1+\langle\xi\rangle^{2 \delta}|z|^{2}\right)^{-M}\left(1+\langle\xi\rangle^{2 \delta}\left(-\Delta_{\zeta}\right)\right)^{M} \partial_{\xi}^{\alpha} \partial_{x}^{\beta} \sigma(x+z, \xi, \xi+s \zeta)
$$


We also have that

$$
\begin{aligned}
\mid(1 & \left.+\langle\xi\rangle^{2 \delta}\left(-\Delta_{\zeta}\right)\right)^{M} \partial_{\xi}^{\alpha} \partial_{x}^{\beta} \partial_{z}^{\gamma} \sigma(x+z, \xi, \xi+s \zeta) \mid \\
\lesssim & \sum_{|\lambda|<M}\langle\xi\rangle^{2 \delta|\lambda|}\left(\partial_{1}^{\beta+\gamma} \partial_{2}^{\alpha} \partial_{3}^{2 \lambda} c_{2}\right)(x+z, \xi, \xi+s \zeta) \\
& +\sum_{|\lambda|<M}\langle\xi\rangle^{2 \delta|\lambda|}\left(\partial_{1}^{\beta+\gamma} \partial_{3}^{2 \lambda+\alpha} c_{2}\right)(x+z, \xi, \xi+s \zeta) \\
& \lesssim \sum_{|\lambda|<M}\langle\xi\rangle^{2 \delta|\lambda|}\langle\xi\rangle^{m+\delta|\beta|+\delta|\gamma|-\rho|\alpha|}\langle\xi+s \zeta\rangle^{m^{\prime}-2|\lambda|} \\
& +\sum_{|\lambda|<M}\langle\xi\rangle^{2 \delta|\lambda|}\langle\xi\rangle^{m+\delta|\beta|+\delta|\gamma|}\langle\xi+s \zeta\rangle^{m^{\prime}-2|\lambda|-|\alpha|} .
\end{aligned}
$$

Divide the domain of integration in $\zeta$ in (14) into three pieces $\mathbf{A}:=\left\{|\zeta| \leq\langle\xi\rangle^{\delta} / 2\right\}$, $\mathbf{B}:=\left\{\langle\xi\rangle^{\delta} / 2 \leq|\zeta| \leq\langle\xi\rangle / 2\right\}$ and $\mathbf{C}:=\{|\zeta| \geq\langle\xi\rangle / 2\}$.

We observe that for $s \in[0,1],|\zeta| \leq\langle\xi\rangle / 2$ we get

$$
|\langle\xi+s \zeta\rangle-\langle\xi\rangle|=\left|s \int_{0}^{1} \sum_{k=1}^{n} \zeta_{j} \partial_{\xi_{j}}\langle\xi+t s \zeta\rangle \mathrm{d} t\right| \leq \frac{\langle\xi\rangle}{2}
$$

since $\left|\partial_{\xi_{j}}\langle\xi+t s \zeta\rangle\right| \leq 1$. This implies that for $s \in[0,1],|\zeta| \leq\langle\xi\rangle / 2$ we have

$$
\frac{\langle\xi\rangle}{2} \leq\langle\xi+s \zeta\rangle \leq \frac{3\langle\xi\rangle}{2}
$$

Moreover (16) and (17) also yield that in $\mathbf{A} \cup \mathbf{B}$ we have

$$
\left|R_{S}(x, \xi, z, \zeta)\right| \lesssim\left(1+\langle\xi\rangle^{2 \delta}|z|^{2}\right)^{-M}\langle\xi\rangle^{m+m^{\prime}-\rho|\alpha|+\delta|\beta|}
$$

\section{Hence}

$$
\begin{aligned}
\left|\int_{\mathbf{A}} \int_{\mathbb{R}^{n}} e^{-i z \cdot \zeta} R_{S} \mathrm{~d} z đ \zeta\right| & \lesssim\langle\xi\rangle^{m+m^{\prime}-\rho|\alpha|+\delta|\beta|-\delta n} \int_{\mathbf{A}} \int_{\mathbb{R}^{n}}\left(1+|u|^{2}\right)^{-M} \mathrm{~d} u đ \zeta \\
& \lesssim\langle\xi\rangle^{m+m^{\prime}-\rho|\alpha|+\delta|\beta|} .
\end{aligned}
$$

Next we observe that since

$$
\left|\partial_{z}^{\gamma}\left(\left(1+\langle\xi\rangle^{2 \delta}|z|^{2}\right)^{-M}\right)\right| \lesssim\langle\xi\rangle^{\delta|\gamma|}\left(1+\langle\xi\rangle^{2 \delta}|z|^{2}\right)^{-M}
$$

one has

$$
\left|\left(-\Delta_{z}\right)^{M} R_{S}\right| \lesssim\langle\xi\rangle^{m+m^{\prime}-\rho|\alpha|+\delta|\beta|+2 M \delta}\left(1+\langle\xi\rangle^{2 \delta}|z|^{2}\right)^{-M} .
$$


Therefore

$$
\begin{aligned}
& \left|\int_{\mathbf{B}} \int_{\mathbb{R}^{n}} e^{-i z \cdot \zeta} R_{S} \mathrm{~d} z \mathrm{~d} \zeta\right|=\left.\left|\int_{\mathbf{B}} \int_{\mathbb{R}^{n}} e^{-i z \cdot \zeta}\right| \zeta\right|^{-2 M}\left(-\Delta_{z}\right)^{M} R_{S} \mathrm{~d} z \mathrm{~d} \zeta \mid \\
& \quad \lesssim\langle\xi\rangle^{m+m^{\prime}-\rho|\alpha|+\delta|\beta|+(2 M-n) \delta} \int_{\mathbf{B}}|\zeta|^{-2 M} \int_{\mathbb{R}^{n}}\left(1+|u|^{2}\right)^{-M} \mathrm{~d} u \mathrm{~d} \zeta \\
& \quad \lesssim\langle\xi\rangle^{m+m^{\prime}-\rho|\alpha|+\delta|\beta|} .
\end{aligned}
$$

On the set $\mathbf{C}$ we have $\langle\xi\rangle \leq 2|\zeta|$ and for all $s \in[0,1]$ that

$$
\langle\xi+s \zeta\rangle \leq\langle\xi\rangle+|\zeta| \leq 3|\zeta|
$$

Hence the definition of $R_{S}$ in (15) and (16), (18), yield that

$$
\left|\left(-\partial_{z}^{\gamma}\right) R_{S}\right| \lesssim|\zeta|^{\max \left(m^{\prime}, 0\right)+\delta|\beta|+2 M \delta+\delta|\gamma|}\langle\xi\rangle^{m}\left(1+\langle\xi\rangle^{2 \delta}|z|^{2}\right)^{-M} .
$$

Therefore integrating by parts and choosing $N$ so large that

$$
\max \left(m^{\prime}, 0\right)+\delta|\beta|+2 M \delta-2 N(1-\delta)<-n
$$

and

$m+\delta|\beta|+\max \left(m^{\prime}, 0\right)+2 M \delta-2 N(1-\delta)+(1-\delta) n \leq m+m^{\prime}-\rho|\alpha|+\delta|\beta|$

(observe once again that $\delta<1$ ), we obtain

$$
\begin{aligned}
\left|\int_{\mathbf{C}} \int_{\mathbb{R}^{n}} e^{-i z \cdot \zeta} R_{S} \mathrm{~d} z \mathrm{~d} \zeta\right| & =\left.\left|\int_{\mathbf{C}} \int_{\mathbb{R}^{n}} e^{-i z \cdot \zeta}\right| \zeta\right|^{-2 N}\left(-\Delta_{z}\right)^{N} R_{S} \mathrm{~d} z \mathrm{~d} \zeta \mid \\
& \lesssim\langle\xi\rangle^{m-n \delta} \int_{\mathbf{C}}|\zeta|^{\max \left(m^{\prime}, 0\right)+\delta|\beta|+2 M \delta-2 N(1-\delta)} \mathrm{d} \zeta \\
& \lesssim\langle\xi\rangle^{m+m^{\prime}-\rho|\alpha|+\delta|\beta|} .
\end{aligned}
$$

This concludes the proof.

\section{Decomposition of the FIOs}

In connection to the study of the $L^{p}$-regularity of FIOs, based on an idea of C. Fefferman [12], Seeger, Sogge and Stein [23] introduced a second dyadic decomposition superimposed on a preliminary Littlewood-Paley decomposition.

Note that, since we are dealing with FIOs with amplitudes in general Hörmander classes, the constructions in [23] have to be generalised to this setting. Here we follow the rendition of the Seeger-Sogge-Stein decomposition, as was given in Dos Santos Ferreira-Staubach [11]. 
To start, one considers an FIO $T_{a}^{\varphi}$ with amplitude $a(x, \xi) \in S_{\rho, \delta}^{m}\left(\mathbb{R}^{n}\right), 0<\rho \leq 1$, $0 \leq \delta<1, m \in \mathbb{R}$ and the SND phase $\varphi \in \Phi^{2}$ and its Littlewood-Paley decomposition

$$
T_{a}^{\varphi}=\sum_{j=0}^{\infty} T_{a}^{\varphi} \psi_{j}(D)=: \sum_{j=0}^{\infty} T_{j},
$$

where the kernel $K_{j}$ of $T_{j}$ is given by

$$
\begin{aligned}
K_{j}(x, y) & =\int_{\mathbb{R}^{n}} e^{i \varphi(x, \xi)-i y \cdot \xi} \psi_{j}(\xi) a(x, \xi) đ \xi \\
& =2^{j n \rho} \int_{\mathbb{R}^{n}} e^{i 2^{j \rho} \varphi(x, \xi)-i 2^{j \rho} y \cdot \xi} \psi_{j}\left(2^{j \rho} \xi\right) a\left(x, 2^{j \rho} \xi\right) \mathrm{d} \xi
\end{aligned}
$$

Here each $\psi_{j}$ is supported in a dyadic shell $\left\{2^{j-1} \leq|\xi| \leq 2^{j+1}\right\}$ (as in Definition 2.1) so the $\xi$-support of the integrand in the second integral is

$$
A_{j}:=\left\{\xi \in \mathbb{R}^{n}: 2^{j(1-\rho)-1} \leq|\xi| \leq 2^{j(1-\rho)+1}\right\} .
$$

The shells $A_{j}$ will in turn be decomposed into truncated cones using the following construction:

Definition 5.1 For each $j \in \mathbb{N}$ and $0<\rho \leq 1$, we fix a collection of unit vectors $\left\{\xi_{j}^{\nu}\right\}$ that satisfy the following two conditions.

(i) $\left|\xi_{j}^{\nu}-\xi_{j}^{v^{\prime}}\right| \geq 2^{-j \rho / 2}$, if $v \neq v^{\prime}$.

(ii) If $\xi \in \mathbb{S}^{n-1}$, then there exists a $\xi_{j}^{\nu}$ so that $\left|\xi-\xi_{j}^{\nu}\right|<2^{-j \rho / 2}$.

To do this we take a collection $\left\{\xi_{j}^{\nu}\right\}$ which is maximal with respect to the first property of Definition 5.1.

Let $\Gamma_{j}^{v}$ denote the cone in the $\xi$-space, with the apex at the origin, whose central direction is $\xi_{j}^{\nu}$, i.e.

$$
\Gamma_{j}^{\nu}:=\left\{\xi \in \mathbb{R}^{n}:\left|\frac{\xi}{|\xi|}-\xi_{j}^{\nu}\right| \leq 2 \cdot 2^{-j \rho / 2}\right\}
$$

One also defines

$$
\chi_{j}^{v}:=\frac{\eta_{j}^{\nu}}{\sum_{v} \eta_{j}^{v}}
$$

where

$$
\eta_{j}^{\nu}(\xi):=\phi\left(2^{j \rho / 2}\left(\frac{\xi}{|\xi|}-\xi_{j}^{\nu}\right)\right)
$$

and $\phi$ is a non-negative function in $\mathcal{C}_{c}^{\infty}\left(\mathbb{R}^{n}\right)$ with $\phi(u)=1$ for $|u| \leq 1$ and $\phi(u)=0$ for $|u| \geq 2$. 
Now turning back to the shells (20), we decompose each $A_{j}$ into truncated cones $\Gamma_{j}^{v} \cap A_{j}$ and observe that since the diameter of the set $\Gamma_{j}^{v} \cap\{\xi ;|\xi|=1\}$ is of size $2^{-j \rho / 2}$, using elementary geometry, it is clear that the diameter of the set $\Gamma_{j}^{v} \cap\{\xi ;|\xi|=$ $\left.2^{j(1-\rho)+1}\right\}$ is of size $2^{j(1-\rho)} 2^{-j \rho / 2}$. Therefore, for each truncated cone $\Gamma_{j}^{\nu} \cap A_{j}$ there are $n-1$ directions with length (roughly) equal to $2^{j(1-\rho)} 2^{-j \rho / 2}$ and one direction with length roughly equal to $2^{j(1-\rho)}$ (which is the thickness of $A_{j}$ ). Hence we infer that

$$
\left|\Gamma_{j}^{\nu} \cap A_{j}\right| \sim\left(2^{-j \rho / 2} 2^{j(1-\rho)}\right)^{(n-1)} 2^{j(1-\rho)}=2^{j(n+\rho(1-3 n) / 2)} .
$$

Using this, it also follows that there are $O\left(2^{j \rho(n-1) / 2}\right)$ such truncated cones needed to cover one shell $A_{j}$, and thus there are at most $O\left(2^{j \rho(n-1) / 2}\right)$ elements in the collection $\left\{\xi_{j}^{v}\right\}$, with regard to $\nu$ 's.

For the following lemma and throughout the rest of the paper, we choose the coordinate axes in $\xi$-space such that $\xi_{1}$ is in the direction of $\xi_{j}^{v}$ and $\xi^{\prime}:=\left(\xi_{2}, \ldots, \xi_{n}\right)$ is perpendicular to $\xi_{j}^{v}$.

Lemma 5.2 The functions $\chi_{j}^{v}$ belong to $\mathcal{C}^{\infty}\left(\mathbb{R}^{n} \backslash\{0\}\right)$ and are supported in the cones $\Gamma_{j}^{v}$. They sum to 1 in $v$ :

$$
\sum_{\nu} \chi_{j}^{v}(\xi)=1, \quad j \in \mathbb{N} \text { and } \xi \neq 0
$$

and moreover they satisfy the estimates

$$
\left|\partial_{\xi}^{\alpha} \chi_{j}^{\nu}(\xi)\right| \lesssim 2^{j \rho|\alpha| / 2}|\xi|^{-|\alpha|}
$$

for all multi-indices $\alpha$ and

$$
\left|\partial_{\xi_{1}}^{N} \chi_{j}^{\nu}(\xi)\right| \leq C_{N}|\xi|^{-N}, \text { for } N \geq 1
$$

Proof In proving (23) we note that the argument of $\eta_{j}^{\nu}$ contains a factor of $2^{j \rho / 2}$ followed by a factor that is homogeneous of degree zero. Hence $\alpha$ derivatives yield a factor of $2^{j \rho|\alpha| / 2}$ and a function that is homogeneous of degree $-|\alpha|$. To prove (24) one observes that in the support of $\chi_{j}^{v}$ one can write

$$
\partial_{\xi_{1}}=\partial_{r}+O\left(2^{-j \rho / 2}\right) \cdot \nabla_{\xi},
$$

where $\partial_{r}$ is the radial derivative and $\partial_{r}^{N} \chi_{j}^{v}=0$ since $\chi_{j}^{v}$ is homogeneous of degree zero.

We will split the phase $\varphi(x, \xi)-y \cdot \xi$ into two different pieces, $\left(\nabla_{\xi} \varphi\left(x, \xi_{j}^{v}\right)-y\right) \cdot \xi$ (which is linear in $\xi$ ), and $\varphi(x, \xi)-\nabla_{\xi} \varphi\left(x, \xi_{j}^{\nu}\right) \cdot \xi$. The following lemma yields an estimate for the nonlinear second piece. 
Lemma 5.3 For $j, v \geq 1$, define

$$
h_{j}^{\nu}(x, \xi):=\varphi(x, \xi)-\nabla_{\xi} \varphi\left(x, \xi_{j}^{\nu}\right) \cdot \xi
$$

Then for $0<\rho \leq 1$ and for $\xi$ in $A_{j} \cap \Gamma_{j}^{v}$ (see (20) and (21)), one has that

$$
\left|\partial_{\xi^{\prime}}^{\alpha} h_{j}^{\nu}(x, \xi)\right| \lesssim\left\{\begin{array}{ll}
\left|\xi^{\prime}\right||\xi|^{-1} & \text { if }|\alpha|=1 \\
|\xi|^{1-|\alpha|} & \text { if }|\alpha| \geq 2
\end{array} \lesssim 2^{-j \rho / 2}\right.
$$

and for $N \geq 1$

$$
\left|\partial_{\xi_{1}}^{N} h_{j}^{v}(x, \xi)\right| \lesssim\left|\xi^{\prime}\right|^{2}|\xi|^{-N-1} \lesssim 2^{-j \rho}
$$

Proof The proof is based on simple Taylor expansions and homogeneity considerations, see [24, p. 407]. Also observe that $\left|\xi^{\prime}\right| \lesssim 2^{j(1-3 \rho / 2)}$.

Remark 5.4 Note that by the previous lemma, one has the estimate

$$
\left|\nabla_{\xi} h_{j}^{v}(x, \xi)\right| \lesssim 2^{-j \rho / 2},
$$

for all $\xi \in \Gamma_{j}^{v}$, due to the fact that $\nabla_{\xi} h_{j}^{v}(x, \xi)$ is homogeneous of degree zero in $\xi$.

In [23] the authors define an "influence set" associated to the SND phase function $\varphi$. We have to make a similar definition but it has to be fitted to the more general classes of amplitudes that we are considering here. To this end we have

Definition 5.5 Assume that $\varphi$ is an $S N D$ phase function in the class $\Phi^{2}$, and let $\bar{y} \in \mathbb{R}^{n}$ be the centre of a ball $B$ with radius $r<1$, and $\rho \in(0,1]$. Define for $j, v \geq 1$,

$$
\tilde{R}_{j}^{v}:=\left\{y \in \mathbb{R}^{n}:|y-\bar{y}| \leq c 2^{-\rho j}, \quad\left|\pi_{j}^{v}(y-\bar{y})\right| \leq c 2^{-\rho j / 2}\right\},
$$

where $\pi_{j}^{v}$ is the orthogonal projection in the direction $\xi_{j}^{v}$ and $c$ is a large constant depending on the size of the Hessian matrix of $\varphi$ but independent of $j$, to be specified later. We also define $R_{j}^{v}$ as the preimage of $\tilde{R}_{j}^{v}$ under the mapping $x \rightarrow \nabla_{\xi} \varphi\left(x, \xi_{j}^{v}\right)$, i.e.

$$
R_{j}^{v}:=\left\{x \in \mathbb{R}^{n}:\left|\nabla_{\xi} \varphi\left(x, \xi_{j}^{\nu}\right)-\bar{y}\right| \leq c 2^{-\rho j}, \quad\left|\pi_{j}^{\nu}\left(\nabla_{\xi} \varphi\left(x, \xi_{j}^{v}\right)-\bar{y}\right)\right| \leq c 2^{-\rho j / 2}\right\} .
$$

Now set

$$
B^{*}=\bigcup_{2^{-j} \leq r} \bigcup_{v} R_{j}^{v}
$$

and recall from the discussion following Definition 5.1 that the number of $v$ 's in the union above is $O\left(2^{j \rho(n-1) / 2}\right)$.

In this connection we have the following estimates. 
Lemma 5.6 Let $\bar{y} \in \mathbb{R}^{n}$ be the centre of a ball $B$ with radius $r<1$ and let $B^{*}$ be defined as in (27). Then for $\rho \in(0,1]$

(i) One has the following estimate for the measure of $B^{*}$

$$
\left|B^{*}\right| \lesssim r^{\rho}
$$

(ii) If $x \in \mathbb{R}^{n} \backslash B^{*}, y \in B(\bar{y}, r)$ and the integer $k \geq 1$ is uniquely chosen in such a way that $2^{-(k-1)} \leq r<2^{-k}$, then for $c$ in (26) large enough, we have

$$
2^{j \rho}\left|\left(\nabla_{\xi} \varphi\left(x, \xi_{j}^{\nu}\right)-y\right)_{1}\right|+2^{j \rho / 2}\left|\left(\nabla_{\xi} \varphi\left(x, \xi_{j}^{\nu}\right)-y\right)^{\prime}\right| \gtrsim 2^{(j-k) \rho / 2}, \quad j \geq k .
$$

Proof $(i)$ Since $R_{j}^{v}$ is of size $O\left(2^{-j \rho}\right)$ in the $\xi_{j}^{v}$-direction and $O\left(2^{-j \rho / 2}\right)$ in the other $n-1$ directions, we have for $0<\rho \leq 1$

$$
\left|B^{*}\right| \leq \sum_{2^{-j} \leq r} \sum_{\nu}\left|R_{j}^{v}\right| \lesssim \sum_{2^{-j} \leq r} 2^{j \rho(n-1) / 2} 2^{-j(\rho+\rho(n-1) / 2)} \lesssim r^{\rho} .
$$

(ii) Observe that (28) is equivalent to

$$
2^{(j+k) \rho / 2}\left|\left(\nabla_{\xi} \varphi\left(x, \xi_{j}^{\nu}\right)-y\right)_{1}\right|+2^{k \rho / 2}\left|\left(\nabla_{\xi} \varphi\left(x, \xi_{j}^{\nu}\right)-y\right)^{\prime}\right| \gtrsim 1, \quad j \geq k .
$$

Moreover, it is enough to show that

$$
2^{k \rho}\left|\left(\nabla_{\xi} \varphi\left(x, \xi_{j}^{\nu}\right)-y\right)_{1}\right|+2^{k \rho / 2}\left|\left(\nabla_{\xi} \varphi\left(x, \xi_{j}^{\nu}\right)-y\right)^{\prime}\right| \gtrsim 1 .
$$

Since $\xi_{j}^{v} \in \mathbb{S}^{n-1}$, by Definition 5.1 part $(i i)$, there exists a unit vector $\xi_{k}^{\nu^{\prime}}$ such that

$$
\left|\xi_{j}^{\nu}-\xi_{k}^{\nu^{\prime}}\right|<2^{-k \rho / 2}
$$

Furthermore, since $\mathbb{R}^{n} \backslash B^{*} \subset \mathbb{R}^{n} \backslash R_{k}^{v^{\prime}}$ then the assumption $x \in \mathbb{R}^{n} \backslash B^{*}$ yields that $x \in \mathbb{R}^{n} \backslash R_{k}^{v^{\prime}}$ and therefore by definition (26) we have

$$
2^{k \rho}\left|\pi_{k}^{v^{\prime}}\left(\nabla_{\xi} \varphi\left(x, \xi_{k}^{\nu^{\prime}}\right)-\bar{y}\right)\right|+2^{k \rho / 2}\left|\nabla_{\xi} \varphi\left(x, \xi_{k}^{\nu^{\prime}}\right)-\bar{y}\right| \geq c,
$$

which in turn implies that

$$
2^{k \rho}\left|\left(\nabla_{\xi} \varphi\left(x, \xi_{k}^{v^{\prime}}\right)-\bar{y}\right)_{1}\right|+2^{k \rho / 2}\left|\left(\nabla_{\xi} \varphi\left(x, \xi_{k}^{v^{\prime}}\right)-\bar{y}\right)^{\prime}\right| \geq c / 2
$$

for $c$ sufficiently large. Moreover we also have that

$$
\left|\nabla_{\xi} \varphi\left(x, \xi_{j}^{v}\right)-\nabla_{\xi} \varphi\left(x, \xi_{k}^{v^{\prime}}\right)\right| \lesssim 2^{-k \rho / 2}
$$


Indeed, we observe that since $\varphi \in \Phi^{2}$ one has that $\left|\partial_{\xi \xi}^{2} \varphi(x, \xi)\right| \lesssim|\xi|^{-1}$, for all $x$ and all $\xi \neq 0$. Therefore the mean-value theorem and (30) yield that for some $t \in[0,1]$ and all $k \geq 1$ one has

$$
\begin{aligned}
& \left|\nabla_{\xi} \varphi\left(x, \xi_{j}^{v}\right)-\nabla_{\xi} \varphi\left(x, \xi_{k}^{v^{\prime}}\right)\right| \\
& \quad \leq\left|\xi_{j}^{v}-\xi_{k}^{v^{\prime}}\right|\left|\xi_{j}^{v}+t\left(\xi_{k}^{v^{\prime}}-\xi_{j}^{v}\right)\right|^{-1} \\
& \quad \leq 2^{-k \rho / 2}\left(1-t 2^{-k \rho / 2}\right)^{-1} \leq 2^{-k \rho / 2}\left(1-2^{-\rho / 2}\right)^{-1} \lesssim 2^{-k \rho / 2} .
\end{aligned}
$$

Note that (32) trivially implies

$$
\left|\left(\nabla_{\xi} \varphi\left(x, \xi_{j}^{\nu}\right)-\nabla_{\xi} \varphi\left(x, \xi_{k}^{\nu^{\prime}}\right)\right)^{\prime}\right| \lesssim 2^{-k \rho / 2} .
$$

We also claim that (30) yields

$$
\left|\left(\nabla_{\xi} \varphi\left(x, \xi_{j}^{\nu}\right)-\nabla_{\xi} \varphi\left(x, \xi_{k}^{v^{\prime}}\right)\right) \cdot \xi_{k}^{v^{\prime}}\right| \lesssim 2^{-k \rho}
$$

To see this, using the homogeneity of $\varphi$ (dictated by the $\Phi^{2}$-condition) we have

$$
\begin{aligned}
& \left(\nabla_{\xi} \varphi\left(x, \xi_{j}^{v}\right)-\nabla_{\xi} \varphi\left(x, \xi_{k}^{v^{\prime}}\right)\right) \cdot \xi_{k}^{v^{\prime}} \\
& \quad=\left(\nabla_{\xi} \varphi\left(x, \xi_{j}^{v}\right)-\nabla_{\xi} \varphi\left(x, \xi_{k}^{v^{\prime}}\right)\right) \cdot\left(\xi_{k}^{v^{\prime}}-\xi_{j}^{v}\right)+\varphi\left(x, \xi_{j}^{v}\right)-\nabla_{\xi} \varphi\left(x, \xi_{k}^{v^{\prime}}\right) \cdot \xi_{j}^{v} \\
& \quad=\left(\nabla_{\xi} \varphi\left(x, \xi_{j}^{v}\right)-\nabla_{\xi} \varphi\left(x, \xi_{k}^{v^{\prime}}\right)\right) \cdot\left(\xi_{k}^{v^{\prime}}-\xi_{j}^{v}\right)+h_{k}^{v^{\prime}}\left(x, \xi_{j}^{v}\right),
\end{aligned}
$$

where

$$
h_{k}^{\nu^{\prime}}(x, \xi):=\varphi(x, \xi)-\nabla_{\xi} \varphi\left(x, \xi_{k}^{v^{\prime}}\right) \cdot \xi
$$

Moreover, by (25) one has the estimate

$$
\left|\left(\nabla_{\xi} h_{k}^{v^{\prime}}\right)(x, \xi)\right| \leq 2^{-k \rho / 2}
$$

for all $\xi \in \Gamma_{k}^{v^{\prime}}=\left\{\xi ;\left|\frac{\xi}{|\xi|}-\xi_{k}^{v^{\prime}}\right|<2 \cdot 2^{-k \rho / 2}\right\}$ (which is a cone with vertex at the origin and central direction $\xi_{k}^{v^{\prime}}$ ). Recalling that $\xi_{j}^{v}$ is in $\mathbb{S}^{n-1},(30)$ shows that $\xi_{j}^{v}$ also belongs to the cone $\Gamma_{k}^{v^{\prime}}$, and so for all $t \in[0,1]$, the expression $\xi_{j}^{v}+t\left(\xi_{k}^{v^{\prime}}-\xi_{j}^{v}\right)$ which represents the line segment joining $\xi_{j}^{v}$ and $\xi_{k}^{v^{\prime}}$ belongs to $\Gamma_{k}^{v^{\prime}}$, due to the convexity of the cone. Therefore, (35) yields that for all $t \in[0,1]$

$$
\left|\left(\nabla_{\xi} h_{k}^{v^{\prime}}\right)\left(x, \xi_{j}^{v}+t\left(\xi_{k}^{v^{\prime}}-\xi_{j}^{\nu}\right)\right)\right| \lesssim 2^{-k \rho / 2}
$$

Now we also observe that $h_{k}^{\nu^{\prime}}\left(x, \xi_{k}^{\nu^{\prime}}\right)=0$. Hence, using the mean-value theorem and (30), one readily sees that $h_{k}^{\nu^{\prime}}\left(x, \xi_{j}^{\nu}\right)=O\left(2^{-k \rho}\right)$. For the term $\left(\nabla_{\xi} \varphi\left(x, \xi_{j}^{v}\right)-\right.$ $\left.\nabla_{\xi} \varphi\left(x, \xi_{k}^{v^{\prime}}\right)\right) \cdot\left(\xi_{k}^{\nu^{\prime}}-\xi_{j}^{\nu}\right)$ we just use the Cauchy-Schwarz inequality, (30) and (32), which concludes the proof of (34). 
Now having (33) we claim that

$$
\left|\left(\nabla_{\xi} \varphi\left(x, \xi_{j}^{\nu}\right)-\nabla_{\xi} \varphi\left(x, \xi_{k}^{v^{\prime}}\right)\right)_{1}\right| \lesssim 2^{-k \rho}
$$

This is because our initial convention that $\xi_{j}^{v}$ lies along the $\xi_{1}$-axis, the triangle inequality, the Cauchy-Schwarz inequality, (30), (32) and (34), yield that

$$
\begin{aligned}
& \left|\left(\nabla_{\xi} \varphi\left(x, \xi_{j}^{\nu}\right)-\nabla_{\xi} \varphi\left(x, \xi_{k}^{\nu^{\prime}}\right)\right)_{1}\right|=\left|\left(\nabla_{\xi} \varphi\left(x, \xi_{j}^{\nu}\right)-\nabla_{\xi} \varphi\left(x, \xi_{k}^{\nu^{\prime}}\right)\right) \cdot \xi_{j}^{\nu}\right|= \\
& \quad=\left|\left(\nabla_{\xi} \varphi\left(x, \xi_{j}^{\nu}\right)-\nabla_{\xi} \varphi\left(x, \xi_{k}^{v^{\prime}}\right)\right)\left(\xi_{j}^{\nu}-\xi_{k}^{\nu^{\prime}}\right)+\left(\nabla_{\xi} \varphi\left(x, \xi_{j}^{\nu}\right)-\nabla_{\xi} \varphi\left(x, \xi_{k}^{\nu^{\prime}}\right)\right) \cdot \xi_{k}^{v^{\prime}}\right| \\
& \quad \leq\left|\nabla_{\xi} \varphi\left(x, \xi_{j}^{\nu}\right)-\nabla_{\xi} \varphi\left(x, \xi_{k}^{\nu^{\prime}}\right)\right|\left|\xi_{j}^{\nu}-\xi_{k}^{\nu^{\prime}}\right|+\left|\left(\nabla_{\xi} \varphi\left(x, \xi_{j}^{\nu}\right)-\nabla_{\xi} \varphi\left(x, \xi_{k}^{v^{\prime}}\right)\right) \cdot \xi_{k}^{\nu^{\prime}}\right| \\
& \quad \lesssim 2^{-k \rho} .
\end{aligned}
$$

Finally to show (29) (which as we mentioned above implies the desired estimate (28)), we use the triangle inequality, (31), (33) and (36) to obtain

$$
\begin{aligned}
& 2^{k \rho}\left|\left(\nabla_{\xi} \varphi\left(x, \xi_{j}^{\nu}\right)-y\right)_{1}\right|+2^{k \rho / 2}\left|\left(\nabla_{\xi} \varphi\left(x, \xi_{j}^{\nu}\right)-y\right)^{\prime}\right| \\
& =2^{k \rho}\left|\left(\nabla_{\xi} \varphi\left(x, \xi_{j}^{v}\right)-\bar{y}-(y-\bar{y})\right)_{1}\right|+2^{k \rho / 2}\left|\left(\nabla_{\xi} \varphi\left(x, \xi_{j}^{v}\right)-\bar{y}-(y-\bar{y})\right)^{\prime}\right| \\
& \geq 2^{k \rho}\left|\left(\nabla_{\xi} \varphi\left(x, \xi_{j}^{\nu}\right)-\bar{y}\right)_{1}\right|-2^{k \rho}\left|y_{1}-\bar{y}_{1}\right|+2^{k \rho / 2}\left|\left(\nabla_{\xi} \varphi\left(x, \xi_{j}^{\nu}\right)-\bar{y}\right)^{\prime}\right| \\
& -2^{k \rho / 2}\left|y^{\prime}-\bar{y}^{\prime}\right| \\
& \geq 2^{k \rho}\left|\left(\nabla_{\xi} \varphi\left(x, \xi_{j}^{\nu}\right)-\bar{y}\right)_{1}\right|+2^{k \rho / 2}\left|\left(\nabla_{\xi} \varphi\left(x, \xi_{j}^{\nu}\right)-\bar{y}\right)^{\prime}\right|-2^{k \rho} 2^{1-k}-2^{k \rho / 2} 2^{1-k} \\
& =2^{k \rho}\left|\left(\nabla_{\xi} \varphi\left(x, \xi_{j}^{\nu}\right)-\nabla_{\xi} \varphi\left(x, \xi_{k}^{v^{\prime}}\right)+\nabla_{\xi} \varphi\left(x, \xi_{k}^{v^{\prime}}\right)-\bar{y}\right)_{1}\right| \\
& +2^{k \rho / 2}\left|\left(\nabla_{\xi} \varphi\left(x, \xi_{j}^{\nu}\right)-\nabla_{\xi} \varphi\left(x, \xi_{k}^{\nu^{\prime}}\right)+\nabla_{\xi} \varphi\left(x, \xi_{k}^{\nu^{\prime}}\right)-\bar{y}\right)^{\prime}\right|-2^{k \rho} 2^{1-k} \\
& -2^{k \rho / 2} 2^{1-k} \\
& \geq 2^{k \rho}\left|\left(\nabla_{\xi} \varphi\left(x, \xi_{k}^{v^{\prime}}\right)-\bar{y}\right)_{1}\right|-2^{k \rho}\left|\left(\nabla_{\xi} \varphi\left(x, \xi_{j}^{\nu}\right)-\nabla_{\xi} \varphi\left(x, \xi_{k}^{v^{\prime}}\right)\right)_{1}\right| \\
& +2^{k \rho / 2}\left|\left(\nabla_{\xi} \varphi\left(x, \xi_{k}^{\nu^{\prime}}\right)-\bar{y}\right)^{\prime}\right|-2^{k \rho / 2}\left|\left(\nabla_{\xi} \varphi\left(x, \xi_{j}^{\nu}\right)-\nabla_{\xi} \varphi\left(x, \xi_{k}^{\nu^{\prime}}\right)\right)^{\prime}\right| \\
& -2^{k \rho} 2^{1-k}-2^{k \rho / 2} 2^{1-k} \\
& \geq 2^{k \rho}\left|\left(\nabla_{\xi} \varphi\left(x, \xi_{k}^{\nu^{\prime}}\right)-\bar{y}\right)_{1}\right|+2^{k \rho / 2}\left|\left(\nabla_{\xi} \varphi\left(x, \xi_{k}^{\nu^{\prime}}\right)-\bar{y}\right)^{\prime}\right| \\
& -2^{k \rho} A 2^{-k}-2^{k \rho / 2} C 2^{-k / 2}-2^{k \rho} 2^{1-k}-2^{k \rho / 2} 2^{1-k} \\
& \geq \frac{c}{2}-2^{k \rho} A 2^{-k}-C 2^{k \rho / 2} 2^{-k / 2}-2^{k \rho} 2^{1-k}-2^{k \rho / 2} 2^{1-k} \\
& =\frac{c}{2}-A 2^{-k(1-\rho)}-C 2^{-k(1-\rho) / 2}-2^{1-k(1-\rho)}-2^{1-k(1-\rho / 2)} \\
& \geq \frac{c}{2}-A-C-4 \text {, }
\end{aligned}
$$

where the constant $A$ stems from estimate (36) and $C$ from (33). Therefore picking $c$ large enough we obtain (28). 


\section{$6 L^{p}$-results}

In this section we prove our main $L^{p}$-boundedness results for FIOs with general Hörmander-class amplitudes. This generalizes the results of Seeger-Sogge-Stein in two ways. First of all this is a global regularity result and opposed to the local one in [23]. Second, we consider all possible values of $\rho$ 's and $\delta$ 's as opposed to just $\rho \in[1 / 2,1]$ and $\delta=1-\rho$.

Remark 6.1 We note that in what follows we can confine ourselves to the case of amplitudes $a(x, \xi)$ that vanish in a neighbourhood of the $\xi=0$. To see this we take $\psi_{0} \in \mathcal{C}_{c}^{\infty}\left(\mathbb{R}^{n}\right)$ be equal to 1 on $B(0,1)$ and have its support in $B(0,2)$, and split the amplitude into the pieces $a(x, \xi) \psi_{0}(\xi)+a(x, \xi)\left(1-\psi_{0}(\xi)\right)$ and observe that the first term is in $S_{1,0}^{m}$ for all $m$. Thus as was shown in [11, Theorem 1.18], the FIO with amplitude $a(x, \xi) \psi_{0}(\xi)$ is an $L^{p}$-bounded operator for $1 \leq p \leq \infty$. This means that our analysis can be concentrated on the amplitude $a(x, \xi)\left(1-\psi_{0}(\xi)\right)$ which belongs to $S_{\rho, \delta}^{m}\left(\mathbb{R}^{n}\right)$ and vanishes near $\xi=0$.

\subsection{Exotic amplitudes}

We start by proving the $L^{p}$-boundedness of exotic FIOs with amplitudes in $a \in$ $S_{\rho, \delta}^{m}\left(\mathbb{R}^{n}\right)$ with $\rho=0,0 \leq \delta<1$.

Proposition 6.2 Let $n \geq 1,0 \leq \delta<1, a \in S_{0, \delta}^{m}\left(\mathbb{R}^{n}\right)$, and assume that $\varphi \in \Phi^{2}$ is SND. Then for

$$
m=-n\left|\frac{1}{p}-\frac{1}{2}\right|-\frac{n \delta}{2}
$$

the FIO $T_{a}^{\varphi}$ is $L^{p}$-bounded for $1<p<\infty$.

Proof Using the discussion in Sect. 3, we shall from now on assume that $T_{a}^{\varphi}$ is of the form

$$
T f(x):=\int_{\mathbb{R}^{n}} a(x, \xi) e^{i(\theta(x, \xi)+x \cdot \xi)} \widehat{f}(\xi) \mathrm{d} \xi,
$$

where $\theta \in \Phi^{1}$ and $\theta(x, \xi)+x \cdot \xi$ is SND.

Since the result has already been proven for the case when $p=2$ (see Proposition 2.16) it only remains to show that $T$ and its adjoint map $\mathscr{H}^{p}\left(\mathbb{R}^{n}\right)$ to $L^{p}\left(\mathbb{R}^{n}\right)$ continuously, for some $p<1$, and thereafter interpolate these with the $L^{2}$-boundedness (see [6] for interpolation of Hardy spaces).

Due to the atomic decomposition in Definition 2.6 of an element of $\mathscr{H}^{p}\left(\mathbb{R}^{n}\right)$, we would need to show that

$$
\int_{\mathbb{R}^{n}}|T \mathfrak{a}(x)|^{p} \mathrm{~d} x
$$

is uniformly bounded for every $\mathscr{H}^{p}$-atom $\mathfrak{a}$, where the atom is supported in the ball $B:=B(\bar{y}, r)$. To prove the assertion in the case $2<p<\infty$ we also need the uniform 
boundedness of (38) for the adjoint operator $T^{*}$. However since the proof is almost identical to the case of $T$, we confine ourselves to this case.

We split $\mathbb{R}^{n}$ into $2 B$ and $\mathbb{R}^{n} \backslash 2 B$ and start with the case of $2 B$ (note that $2 B$ is ball $B(\bar{y}, 2 r)$. By Hölder's inequality and the $L^{2}$-boundedness of $T$, we have

$$
\begin{aligned}
\|T \mathfrak{a}\|_{L^{p}(2 B)} & \lesssim\|T \mathfrak{a}\|_{L^{2}(2 B)}\|1\|_{L^{2 p /(2-p)}(2 B)} \lesssim\|\mathfrak{a}\|_{L^{2}\left(\mathbb{R}^{n}\right)} r^{n(2-p) / 2 p} \\
& \lesssim r^{n(p-2) / 2 p} r^{n(2-p) / 2 p}=1 .
\end{aligned}
$$

We proceed to the boundedness of $\|T \mathfrak{a}\|_{L^{p}\left(\mathbb{R}^{n} \backslash 2 B\right)}$. Now we consider what we call a "generic Littlewood-Paley piece" of the operator $T$ given by (37). The generic Littlewood-Paley piece is the FIO with the amplitude $a_{j}$, where $a_{j}(x, \xi):=$ $a(x, \xi) \psi_{j}(\xi)$, and $j \geq 1$ due to Remark 6.1, and the phase function $x \cdot \xi+\theta(x, \xi)$. We denote this operator by $S_{j}$ and note that the integral kernel of $S_{j}$ is given by

$$
K_{j}(x, y):=\int_{\mathbb{R}^{n}} a_{j}(x, \xi) e^{i \theta(x, \xi)+i(x-y) \cdot \xi} \mathrm{d} \xi
$$

We claim that

$$
\left\|(x-y)^{\alpha} \partial_{y}^{\beta} K_{j}(x, y)\right\|_{L_{x}^{2}\left(\mathbb{R}^{n}\right)} \lesssim 2^{j(|\beta|+m+n / 2+n \delta / 2)},
$$

for all multi-indices $\alpha$ and $\beta$. Since differentiating (40) $\beta$ times in $y$ will only introduce factors of the size $2^{j|\beta|}$, it is enough to establish (41) for $\beta=0$. Now the global $L^{2}$ boundedness (41) of the kernel can be formulated as the $L^{2}$-boundedness of a kernel of the form

$$
\tilde{K}_{j}^{\alpha}(x, x-y):=\int_{\mathbb{R}^{n}} a_{j}(x, \xi)(x-y)^{\alpha} e^{i \theta(x, \xi)+i(x-y) \cdot \xi} \mathrm{d} \xi .
$$

To this end, take $\Psi_{j}$ as in Definition 2.1, integrate by parts and rewrite

$$
\begin{aligned}
\tilde{K}_{j}^{\alpha}(x, x-y)= & \int_{\mathbb{R}^{n}} a_{j}(x, \xi) e^{i \theta(x, \xi)}(-i)^{|\alpha|} \partial_{\xi}^{\alpha} e^{i(x-y) \cdot \xi} \mathrm{d} \xi \\
= & i^{|\alpha|} \int_{\mathbb{R}^{n}} \partial_{\xi}^{\alpha}\left[a_{j}(x, \xi) e^{i \theta(x, \xi)}\right] e^{i(x-y) \cdot \xi} \Psi_{j}(\xi) \mathrm{d} \xi \\
= & \sum_{\alpha_{1}+\alpha_{2}=\alpha} C_{\alpha_{1}, \alpha_{2}} \int_{\mathbb{R}^{n}} \partial_{\xi}^{\alpha_{1}} a_{j}(x, \xi) \partial_{\xi}^{\alpha_{2}} e^{i \theta(x, \xi)} e^{i(x-y) \cdot \xi} \Psi_{j}(\xi) \mathrm{d} \xi \\
= & \sum_{\substack{\alpha_{1}+\alpha_{2}=\alpha \\
\lambda_{1}+\cdots+\lambda_{r}=\alpha_{2}}} C_{\alpha_{1}, \alpha_{2}, \lambda_{1}, \ldots \lambda_{r}} \int_{\mathbb{R}^{n}} \partial_{\xi}^{\alpha_{1}} a_{j}(x, \xi) \\
& \times \partial_{\xi}^{\lambda_{1}} \theta(x, \xi) \cdots \partial_{\xi}^{\lambda_{r}} \theta(x, \xi) e^{i \theta(x, \xi)} e^{i(x-y) \cdot \xi} \Psi_{j}(\xi) đ \xi \\
= & \sum_{\substack{\alpha_{1}+\alpha_{2}=\alpha \\
\lambda_{1}+\cdots+\lambda_{r}=\alpha_{2}}} C_{\alpha_{1}, \alpha_{2}, \lambda_{1}, \ldots \lambda_{r}} 2^{j(m+n \delta / 2)} \int_{\mathbb{R}^{n}} b_{j}^{\alpha_{1}, \alpha_{2}, \lambda_{1}, \ldots, \lambda_{r}}
\end{aligned}
$$




$$
=: \sum_{\substack{\alpha_{1}+\alpha_{2}=\alpha \\ \lambda_{1}+\cdots+\lambda_{r}=\alpha_{2}}} C_{\alpha_{1}, \alpha_{2}, \lambda_{1}, \ldots \lambda_{r}} 2^{j(m+n \delta / 2)} S_{j}^{\alpha_{1}, \alpha_{2}, \lambda_{1}, \ldots \lambda_{r}}\left(\tau_{-y} \Psi_{j}^{\vee}\right)(x),
$$

where $S_{j}^{\alpha_{1}, \alpha_{2}, \lambda_{1}, \ldots \lambda_{r}}$ is an FIO with the phase function $\theta(x, \xi)+x \cdot \xi$ and amplitude $b_{j}^{\alpha_{1}, \alpha_{2}, \lambda_{1}, \ldots, \lambda_{r}}(x, \xi)$ given by

$$
b_{j}^{\alpha_{1}, \alpha_{2}, \lambda_{1}, \ldots, \lambda_{r}}(x, \xi):=2^{-j(m+n \delta / 2)} \partial_{\xi}^{\alpha_{1}} a_{j}(x, \xi) \partial_{\xi}^{\lambda_{1}} \theta(x, \xi) \ldots \partial_{\xi}^{\lambda_{r}} \theta(x, \xi)
$$

Moreover $\left|\lambda_{j}\right| \geq 1$ and $\tau_{-y}$ is a translation by $-y$.

We observe that $b_{j}^{\alpha_{1}, \alpha_{2}, \lambda_{1}, \ldots, \lambda_{r}}(x, \xi) \in S_{0, \delta}^{-n \delta / 2}\left(\mathbb{R}^{n}\right)$ uniformly in $j$, since $a \in$ $S_{0, \delta}^{m}\left(\mathbb{R}^{n}\right)$ and $\theta \in \Phi^{1}$.

Therefore by Proposition 2.16, $S_{j}^{\alpha_{1}, \alpha_{2}, \lambda_{1}, \ldots \lambda_{r}}$ is an $L^{2}$-bounded FIO, so

$$
\begin{aligned}
\left\|\tilde{K}_{j}^{\alpha}(x, x-y)\right\|_{L_{x}^{2}\left(\mathbb{R}^{n}\right)} & \lesssim \sum_{\substack{\alpha_{1}+\alpha_{2}=\alpha \\
\lambda_{1}+\cdots+\lambda_{r}=\alpha_{2}}} 2^{j(m+n \delta / 2)}\left\|S_{j}^{\alpha_{1}, \alpha_{2}, \lambda_{1}, \ldots \lambda_{r}}\left(\tau_{y} \Psi_{j}^{\vee}\right)\right\|_{L^{2}\left(\mathbb{R}^{n}\right)} \\
& \lesssim 2^{j(m+n \delta / 2)}\left\|\Psi_{j}\right\|_{L^{2}\left(\mathbb{R}^{n}\right)} \lesssim 2^{j(m+n / 2+n \delta / 2)}
\end{aligned}
$$

which proves (41).

Now, the estimate in (41) yields that for any integer $M$, if one sums over $|\alpha| \leq M$,

$$
\left\|(1+|x-y|)^{M} K_{j}(x, y)\right\|_{L_{x}^{2}\left(\mathbb{R}^{n}\right)} \lesssim 2^{j(n / 2+m+n \delta / 2)} .
$$

We now observe that for $t \in[0,1], x \in \mathbb{R}^{n} \backslash 2 B$ and $y \in B$, one has

$$
|x-\bar{y}| \lesssim|x-\bar{y}-t(y-\bar{y})| .
$$

Next we introduce

$$
g(x):=(1+|x-\bar{y}|)^{-M},
$$

where $M>n / q$ and $1 / q=1 / p-1 / 2$. The Hölder and the Minkowski inequalities together with (42) and (43) (with $t=1$ ) yield

$$
\begin{aligned}
\left\|S_{j} \mathfrak{a}\right\|_{L^{p}\left(\mathbb{R}^{n} \backslash 2 B\right)} & =\left\|\int_{B} K_{j}(x, y) \mathfrak{a}(y) \mathrm{d} y\right\|_{L_{x}^{p}\left(\mathbb{R}^{n} \backslash 2 B\right)} \\
& \leq\left\|\frac{1}{g(x)} \int_{B} K_{j}(x, y) \mathfrak{a}(y) \mathrm{d} y\right\|_{L_{x}^{2}\left(\mathbb{R}^{n} \backslash 2 B\right)}\|g\|_{L^{q}\left(\mathbb{R}^{n}\right)} \\
& \lesssim \int_{B}\left\|\frac{1}{g(x)} K_{j}(x, y) \mathfrak{a}(y)\right\|_{L_{x}^{2}\left(\mathbb{R}^{n} \backslash 2 B\right)} \mathrm{d} y
\end{aligned}
$$




$$
\begin{aligned}
& \lesssim \int_{B}|\mathfrak{a}(y)|\left\|(1+|x-y|)^{M} K_{j}(x, y)\right\|_{L_{x}^{2}\left(\mathbb{R}^{n} \backslash 2 B\right)} \mathrm{d} y \\
& \lesssim r^{n-n / p} 2^{j(n / 2+m+n \delta / 2)} \lesssim r^{n-n / p} 2^{j(n-n / p)}
\end{aligned}
$$

since $m=-n(1 / p-1 / 2)-n \delta / 2$.

On the other hand, taking $N:=[n(1 / p-1)]$ (note that $N>n / p-n-1$ ), a Taylor expansion of the kernel at the point $y=\bar{y}$ yields that

$$
\begin{aligned}
K_{j}(x, y)= & \sum_{|\beta| \leq N} \frac{(y-\bar{y})^{\beta}}{\beta !} \partial_{y}^{\beta}\left(K_{j}(x, y)\right)_{\mid y=\bar{y}} \\
& +(N+1) \sum_{|\beta|=N+1} \frac{(y-\bar{y})^{\beta}}{\beta !} \int_{0}^{1}(1-t)^{N} \partial_{y}^{\beta}\left(K_{j}(x, y)\right)_{\mid y=\bar{y}+t(y-\bar{y})} \mathrm{d} t
\end{aligned}
$$

and due to vanishing moments of the atom in Definition 2.6, (iii), we may express the operator as

$$
\begin{aligned}
S_{j} \mathfrak{a}(x)= & (N+1) \sum_{|\beta|=N+1} \int_{B} \int_{0}^{1} \frac{(y-\bar{y})^{\beta}}{\beta !}(1-t)^{N} \\
& \partial_{y}^{\beta}\left(K_{j}(x, y)\right)_{\mid y=\bar{y}+t(y-\bar{y})} \mathfrak{a}(y) \mathrm{d} t \mathrm{~d} y .
\end{aligned}
$$

Noting that $\left|(y-\bar{y})^{\beta}\right| \lesssim r^{N+1}$ and applying the same procedure as above together with estimates (41) and (43), we obtain

$$
\left\|S_{j} \mathfrak{a}\right\|_{L^{p}\left(\mathbb{R}^{n} \backslash 2 B\right)} \lesssim r^{N+1-n / p+n} 2^{j(N+1+m+n / 2+n \delta / 2)} \lesssim r^{N+1+n-n / p} 2^{j(N+1+n-n / p)} .
$$

Now we split the proof in two different cases, namely when the radius $r$ of the support of the atom $\mathfrak{a}$ is less than or greater or equal to one.

For $r \geq 1$, (44) yields that

$$
\|T \mathfrak{a}\|_{L^{p}\left(\mathbb{R}^{n} \backslash 2 B\right)}^{p} \lesssim \sum_{j=1}^{\infty}\left\|S_{j} \mathfrak{a}\right\|_{L^{p}\left(\mathbb{R}^{n} \backslash 2 B\right)}^{p} \lesssim \sum_{j=1}^{\infty} r^{n p-n} 2^{j(n p-n)} \lesssim 1 .
$$

Assume now that $r<1$. Choose $\ell \in \mathbb{Z}_{+}$such that $2^{-\ell-1} \leq r<2^{-\ell}$. Using the facts that $2^{-\ell} \approx r, N+1+n-n / p>0, n-n / p<0$, together with (44) and (45) we conclude that

$$
\begin{aligned}
\|T \mathfrak{a}\|_{L^{p}\left(\mathbb{R}^{n} \backslash 2 B\right)}^{p} & \lesssim \sum_{j=1}^{\ell}\left(r^{N+1+n-n / p} 2^{j(N+1+n-n / p)}\right)^{p}+\sum_{j=\ell+1}^{\infty}\left(r^{n-n / p} 2^{j(n-n / p)}\right)^{p} \\
& \lesssim\left(r^{N+1+n-n / p} 2^{\ell(N+1+n-n / p)}\right)^{p}+\left(r^{n-n / p} 2^{\ell(n-n / p)}\right)^{p} \\
& \approx\left(r^{N+1+n-n / p} r^{-(N+1+n-n / p)}\right)^{p}+\left(r^{n-n / p} r^{-(n-n / p)}\right)^{p}
\end{aligned}
$$


$\approx 1$.

Putting this together with (39), yields the uniform boundedness of (38).

The proof of the adjoint case is identical, except for the fact that (41) becomes

$$
\tilde{K}_{j}^{\alpha}(y, x-y):=\int_{\mathbb{R}^{n}} a_{j}(y, \xi)(x-y)^{\alpha} e^{-i \theta(y, \xi)+i(x-y) \cdot \xi} \mathrm{d} \xi
$$

and when applying $\beta$ derivatives in the $y$-variable the $y$-dependence in both arguments has to be taken into consideration.

It is also evident that Proposition 6.2 yields the $L^{p}$-boundedness of pseudodifferential operators with exotic symbols and thereby completes the investigation in [1].

\subsection{Classical amplitudes}

We proceed by proving a global $L^{p}$-boundedness result for classical FIOs with amplitudes in $a \in S_{\rho, \delta}^{m}\left(\mathbb{R}^{n}\right)$ with $0<\rho \leq 1,0 \leq \delta<1$.

Before doing that, we need the following lemma which provides $\mathscr{H}^{q}-L^{2}$ estimates for FIOs with amplitudes in general Hörmander classes.

Lemma 6.3 Let $m_{0} \leq 0, n \geq 1, \rho \in[0,1], \delta \in[0,1)$ and

$$
m:=m_{0}-n \max \left(0, \frac{\delta-\rho}{2}\right) .
$$

Suppose that $a \in S_{\rho, \delta}^{m}\left(\mathbb{R}^{n}\right)$ and that $a(x, \xi)$ vanishes in a neighborhood of $\xi=0$. Also, let $\varphi$ be an SND phase function in the class $\Phi^{2}$. Then $T_{a}^{\varphi}$, defined in (6), satisfies

$$
\left\|T_{a}^{\varphi} f\right\|_{L^{2}\left(\mathbb{R}^{n}\right)} \lesssim\|f\|_{\mathscr{H}^{2 n /\left(n-2 m_{0}\right)}\left(\mathbb{R}^{n}\right)} .
$$

Also for the adjoint operator one has

$$
\left\|\left(T_{a}^{\varphi}\right)^{*} f\right\|_{L^{2}\left(\mathbb{R}^{n}\right)} \lesssim\|f\|_{\mathscr{H}^{2 n /\left(n-2 m_{0}\right)}\left(\mathbb{R}^{n}\right)} .
$$

Proof Since the operator $T_{a}^{\varphi}(1-\Delta)^{-m_{0} / 2}$ is an FIO with the phase $\varphi$ and an amplitude in $S_{\rho, \delta}^{-n \max (0,(\delta-\rho) / 2)}\left(\mathbb{R}^{n}\right)$ it is $L^{2}$-bounded by Proposition 2.16. This $L^{2}$-boundedness together with the estimates for the Bessel potential operators reformulated in terms of embedding of Triebel-Lizorkin spaces (see [27, Corollary 2.7]) yield

$$
\begin{aligned}
\left\|T_{a}^{\varphi} f\right\|_{L^{2}\left(\mathbb{R}^{n}\right)} & =\left\|T_{a}^{\varphi}(1-\Delta)^{-m_{0} / 2}(1-\Delta)^{m_{0} / 2} f\right\|_{L^{2}\left(\mathbb{R}^{n}\right)} \\
& \lesssim\left\|(1-\Delta)^{m_{0} / 2} f\right\|_{L^{2}\left(\mathbb{R}^{n}\right)} \lesssim\|f\|_{\mathscr{H} q\left(\mathbb{R}^{n}\right)},
\end{aligned}
$$

with $1 / q-1 / 2=-m_{0} / n$, which proves (46). Here observe that the choice of the range of $m_{0}$ implies that $0<q \leq 2$. 
Next we prove (47). By Theorem 4.1 the composition $(1-\Delta)^{-m_{0} / 2} T_{a}^{\varphi}$ is an FIO with the phase $\varphi$ and an amplitude in $S_{\rho, \delta}^{-n \max (0,(\delta-\rho) / 2)}\left(\mathbb{R}^{n}\right)$, and therefore $L^{2}$-bounded. Finally, observing that

$$
\left\|\left(T_{a}^{\varphi}\right)^{*}(1-\Delta)^{-m_{0} / 2}\right\|_{L^{2} \rightarrow L^{2}}=\left\|(1-\Delta)^{-m_{0} / 2} T_{a}^{\varphi}\right\|_{L^{2} \rightarrow L^{2}},
$$

one can proceed as above.

Now we are ready to state and prove our main $L^{p}$-estimate for FIOs with general classical Hörmander-type amplitudes.

Proposition 6.4 Let $n \geq 1, a \in S_{\rho, \delta}^{m}\left(\mathbb{R}^{n}\right), \varphi$ be an SND phase function in the class $\Phi^{2}$ and let $T_{a}^{\varphi}$ be given as in Definition 2.15. For $0<\rho \leq 1,0 \leq \delta<1$ and

$$
m=-(n-\rho)\left|\frac{1}{p}-\frac{1}{2}\right|-n \max \left(0, \frac{\delta-\rho}{2}\right),
$$

the FIO $T_{a}^{\varphi}$ is $L^{p}$-bounded for $1<p<\infty$.

Remark 6.5 In Proposition 6.4 it is not possible to consider the case $\rho=0$ for several reasons. First, the definitions of the rectangles in (26) turn out to be inadequate. Second, the choice of $M$ in the proof of (53) below would not be possible. Third, the choice of $L>n / \rho$ in (68) would be problematic.

Proof of Proposition 6.4 For $n=1$, it is well known that FIOs are special cases of pseudodifferential operators and hence the result follows from the corresponding theory for those operators (see e.g. [24]). Therefore, from now on we concentrate on the case $n \geq 2$. We will initially assume that $a(x, \xi)$ is supported in a fixed compact set in the $x$-variable. This will however be removed later on in the proof. Since the result has already been proven for the case when $p=2$ in Proposition 2.16, the only thing that is left to prove is that $T_{a}^{\varphi}$ and its adjoint map $\mathscr{H}^{1}\left(\mathbb{R}^{n}\right)$ to $L^{1}\left(\mathbb{R}^{n}\right)$ continuously, when $a(x, \xi) \in S_{\rho, \delta}^{m}\left(\mathbb{R}^{n}\right)$ with

$$
m=-\frac{n-\rho}{2}-n \max \left(0, \frac{\delta-\rho}{2}\right)
$$

Due to the atomic decomposition in Definition 2.6 of a member of $\mathscr{H}^{1}\left(\mathbb{R}^{n}\right)$ we need to show that

$$
\int_{\mathbb{R}^{n}}\left|T_{a}^{\varphi} \mathfrak{a}(x)\right| \mathrm{d} x
$$

is uniformly bounded for every $\mathscr{H}^{1}$-atom $\mathfrak{a}$, where the atom is supported in the ball $B\left(x_{0}, r\right)$.

Step 1 - Estimates of $\left\|\mathbf{T}_{\mathbf{a}}^{\prime} \mathfrak{a}\right\|_{\mathbf{L}^{1}\left(\mathbb{R}^{\mathbf{n}}\right)}$ when $\mathbf{r}<\mathbf{1}$

Recalling the set $B^{*}$ in (27), we split (48) into two pieces, namely

$$
\left\|T_{a}^{\varphi} \mathfrak{a}\right\|_{L^{1}\left(\mathbb{R}^{n}\right)}=\left\|T_{a}^{\varphi} \mathfrak{a}\right\|_{L^{1}\left(B^{*}\right)}+\left\|T_{a}^{\varphi} \mathfrak{a}\right\|_{L^{1}\left(\mathbb{R}^{n} \backslash B^{*}\right)}=: \mathrm{I}+\mathrm{II}
$$


Using the first part of Lemma 5.6, the Cauchy-Schwarz inequality, and Lemma 6.3 we can deduce that $I$ is uniformly bounded. Indeed take $\mathfrak{b}=|B|^{1-1 / q} \mathfrak{a}$ with $q=2 n /\left(n-2 m^{\prime}\right)$. Note that since $\|\mathfrak{b}\|_{\mathscr{H}^{q}\left(\mathbb{R}^{n}\right)}=1$, we have

$$
\begin{aligned}
\mathrm{I} & \lesssim r^{\rho / 2}\left\|T_{a}^{\varphi} \mathfrak{a}\right\|_{L^{2}\left(\mathbb{R}^{n}\right)} \lesssim r^{\rho / 2}\|\mathfrak{a}\|_{\mathscr{H}^{2 n /\left(n-2 m^{\prime}\right)\left(\mathbb{R}^{n}\right)}} \\
& =r^{\rho / 2+n / q-n}\|\mathfrak{b}\|_{\mathscr{H}^{2 n /\left(n-2 m^{\prime}\right)}\left(\mathbb{R}^{n}\right)}=r^{\rho / 2+n / q-n}=1,
\end{aligned}
$$

provided that

$$
m^{\prime}=\frac{\rho-n}{2} .
$$

Now we have to deal with the last and most complicated part of the proof, that is the boundedness of II. To do this, we use a partition of unity and decompose $T_{a}^{\varphi}$ as in (19). Recall that for $j \geq 1$ the kernel $K_{j}$ of $T_{j}$ is given by

$$
K_{j}(x, y)=2^{j n \rho} \int_{\mathbb{R}^{n}} e^{i 2^{j \rho} \varphi(x, \xi)-i 2^{j \rho} y \cdot \xi} \psi_{j}\left(2^{j \rho} \xi\right) a\left(x, 2^{j \rho} \xi\right) \mathrm{d} \xi .
$$

By Remark 6.1 it is enough to consider $j \geq 1$. We would first like to prove that

$$
\int_{\mathbb{R}^{n}}\left|\nabla_{y} K_{j}(x, y)\right| \mathrm{d} x \lesssim 2^{j}
$$

which immediately yields

$$
\int_{\mathbb{R}^{n}}\left|K_{j}(x, y)-K_{j}(x, z)\right| \mathrm{d} x \lesssim 2^{j}|y-z| .
$$

Now we make a second dyadic decomposition of $K_{j}$ using Lemma 5.2, where each piece has the form,

$$
K_{j}^{\nu}(x, y)=2^{j n \rho} \int_{\mathbb{R}^{n}} e^{i 2^{j \rho} \varphi(x, \xi)-i 2^{j \rho} y \cdot \xi} \chi_{j}^{\nu}(\xi) \psi_{j}\left(2^{j \rho} \xi\right) a\left(x, 2^{j \rho} \xi\right) \mathrm{d} \xi,
$$

where $\chi_{j}^{\nu}(\xi)$ are as in Lemma 5.2. To justify (50), set

$$
h_{j}^{\nu}(x, \xi):=\varphi(x, \xi)-\xi \cdot \nabla_{\xi} \varphi\left(x, \xi_{j}^{\nu}\right)
$$

and observe that

$$
\nabla_{y} K_{j}^{v}(x, y)=2^{j n \rho} \int_{\mathbb{R}^{n}} e^{i 2^{j \rho} \xi \cdot \nabla_{\xi} \varphi\left(x, \xi_{j}^{\nu}\right)-i 2^{j \rho} y \cdot \xi} b_{j}^{\nu}(x, \xi) đ \xi,
$$

with the vector $b_{j}^{v}(x, \xi)$ given by

$$
b_{j}^{\nu}(x, \xi):=-i 2^{j \rho} \xi a\left(x, 2^{j \rho} \xi\right) \chi_{j}^{\nu}(\xi) \psi_{j}\left(2^{j \rho} \xi\right) e^{i 2^{j \rho} h_{j}^{\nu}(x, \xi)} .
$$


Table 1 The estimates on the size of the factors of $b_{j}^{v}(x, \xi)$ after being acted upon by differential operators. The second sum is taken over all partitions of the multi-index $N_{2}$ and is bounded by $2^{j\left|N_{2}\right| \rho}$

\begin{tabular}{lll}
\hline Factor & $\partial_{\xi_{1}}^{2 N_{1}}$ & $\partial_{\xi}^{2 N_{2}}$ \\
\hline$a\left(x, 2^{j \rho} \xi\right)$ & $2^{j m}$ & $2^{j m}$ \\
$\chi_{j}^{\nu}(\xi)$ & 1 & $2^{j\left|N_{2}\right| \rho}$ \\
$2^{j \rho} \xi \psi_{j}\left(2^{j \rho} \xi\right)$ & $2^{j}$ & $2^{j}$ \\
$e^{i 2^{j \rho} h_{j}^{\nu}(x, \xi)}$ & $\sum_{0 \leq \alpha \leq 2 N_{1}} 2^{j \alpha \rho} 2^{-j \alpha \rho}$ & $\sum_{1} 2^{j \alpha_{1} \rho} 2^{-j \alpha_{1} \rho / 2} \ldots 2^{j \alpha_{\kappa} \rho} 2^{-j \alpha_{\kappa} \rho / 2}$ \\
\hline
\end{tabular}

Note that on the $\xi$-support of each component of $b_{j}^{\nu}(x, \xi)$ one has that $|\xi| \sim 2^{j(1-\rho)}$. Therefore to estimate the size of the derivatives of components of $b_{j}^{v}(x, \xi)$, we use this information and Lemmas 5.2 and 5.3. The estimates have been summarised in Table 1.

Hence if we define the differential operator

$$
L:=\left(1-\partial_{\xi_{1}}^{2}\right)\left(1-2^{-j \rho} \Delta_{\xi^{\prime}}\right)
$$

it is clear that for $N \geq 1$

$$
\begin{aligned}
L^{N} e^{i 2^{j \rho} \xi \cdot \nabla_{\xi} \varphi\left(x, \xi_{j}^{\nu}\right)-i 2^{j \rho} y \cdot \xi}= & e^{i \xi \cdot \nabla_{\xi} \varphi\left(x, \xi_{j}^{\nu}\right)-i y \cdot \xi}\left(1+2^{2 j \rho}\left|\left(\nabla_{\xi} \varphi\left(x, \xi_{j}^{\nu}\right)-y\right)_{1}\right|^{2}\right)^{N} \\
& \times\left(1+2^{j \rho}\left|\left(\nabla_{\xi} \varphi\left(x, \xi_{j}^{\nu}\right)-y\right)^{\prime}\right|^{2}\right)^{N}
\end{aligned}
$$

Finally using the information given in Table 1, we deduce that

$$
\left|L^{N} b_{j}^{v}(x, \xi)\right| \lesssim 2^{j(m+1)}
$$

Now using integration by parts

$$
\begin{aligned}
& \nabla_{y} K_{j}^{\nu}(x, y) \\
& =\frac{2^{j n \rho} \int_{\Gamma_{j}^{\nu} \cap A_{j}} e^{i 2^{j \rho} \xi \cdot \nabla_{\xi} \varphi\left(x, \xi_{j}^{\nu}\right)-i 2^{j \rho} y \cdot \xi} L^{N} b_{j}^{v}(x, \xi) \mathrm{d} \xi}{\left(1+2^{2 j \rho}\left|\left(\nabla_{\xi} \varphi\left(x, \xi_{j}^{v}\right)-y\right)_{1}\right|^{2}\right)^{N}\left(1+2^{j \rho}\left|\left(\nabla_{\xi} \varphi\left(x, \xi_{j}^{v}\right)-y\right)^{\prime}\right|^{2}\right)^{N}},
\end{aligned}
$$

where $\Gamma_{j}^{v} \cap A_{j}$ is the support of $b_{j}^{v}(x, \xi)$ and $A_{j}$ and $\Gamma_{j}^{v}$ were defined in (20) and (21) respectively. Let $\widehat{g}_{j}^{v}$ be a smooth cut-off function that is constantly equal to one on the $\xi$-support of $b_{j}^{v}$ and vanishes outside a compact set which is slightly larger the 
aforementioned $\xi$-support. Now set $\mathbf{t}(x):=\nabla_{\xi} \varphi\left(x, \xi_{j}^{v}\right)$ and define

$$
S_{j, y}^{\nu, N} g_{j}^{\nu}(x):=2^{-j-j m^{\prime}} 2^{j n \rho} \int_{\Gamma_{j}^{\nu}} e^{i 2^{j \rho} x \cdot \xi}\left\{L^{N} b_{j}^{\nu}\left(\mathbf{t}^{-1}(x+y), \xi\right)\right\} \widehat{g}_{j}^{\nu}(\xi) đ \xi .
$$

Then because of (52), the choice of $m^{\prime}$ in (49), and that $\mathbf{t}$ is a diffeomorphism, $S_{j, y}^{\nu, N}$ is a $\Psi$ DO of order $-n \max (0,(\delta-\rho) / 2)$ and hence $L^{2}$-bounded, by Proposition 2.16, uniformly in $y$ and $j$. Observe that $\nabla_{y} K_{j}^{v}(x, y)$ can be rewritten as

$$
\nabla_{y} K_{j}^{\nu}(x, y)=\frac{2^{j+j m^{\prime}}\left(S_{j, y}^{\nu, N} g_{j}^{\nu}\right)\left(\nabla_{\xi} \varphi\left(x, \xi_{j}^{\nu}\right)-y\right)}{\left(1+2^{2 j \rho}\left|\left(\nabla_{\xi} \varphi\left(x, \xi_{j}^{\nu}\right)-y\right) 1\right|^{2}\right)^{N}\left(1+2^{j \rho}\left|\left(\nabla_{\xi} \varphi\left(x, \xi_{j}^{\nu}\right)-y\right)^{\prime}\right|^{2}\right)^{N}}
$$

Now using the compact $x$-support, Cauchy-Schwarz inequality and that $\mathbf{t}(x)$ is a diffeomorphism, we have

$$
\begin{aligned}
& \left(\int_{\mathbb{R}^{n}}\left|\nabla_{y} K_{j}^{\nu}(x, y)\right| \mathrm{d} x\right)^{2} \\
& \quad \lesssim \int_{\mathbb{R}^{n}} \frac{2^{2 j+2 j m^{\prime}}\left\|S_{j, y}^{\nu, N} g_{j}^{\nu}\right\|_{L^{2}\left(\mathbb{R}^{n}\right)}^{2}}{\left(1+2^{2 j \rho}\left|\left(\nabla_{\xi} \varphi\left(x, \xi_{j}^{\nu}\right)-y\right)\right|^{2}\right)^{2 N}\left(1+2^{j \rho}\left|\left(\nabla_{\xi} \varphi\left(x, \xi \xi_{j}^{\nu}\right)-y\right)^{\prime}\right|^{2}\right)^{2 N}} \mathrm{~d} x \\
& \quad \lesssim 2^{2 j+2 j m^{\prime}-j(\rho+(n-1) \rho / 2)}\left\|g_{j}^{\nu}\right\|_{L^{2}\left(\mathbb{R}^{n}\right)}^{2} \lesssim 2^{2 j+2 j m^{\prime}-j(n+1) \rho / 2+j(n+\rho(1-3 n) / 2)} \\
& \quad \lesssim 2^{j(2+\rho-2 n \rho)},
\end{aligned}
$$

where recalling (22) we have that $\left|\Gamma_{j}^{\nu} \cap A_{j}\right| \sim 2^{j(n+\rho(1-3 n) / 2)}$. Therefore, summing in $v$ and observing that since there are roughly $2^{j \rho(n-1) / 2}$ terms involved, we obtain

$$
\int_{\mathbb{R}^{n}}\left|\nabla_{y} K_{j}(x, y)\right| \mathrm{d} x \leq \sum_{\nu} 2^{j(2+\rho-2 n \rho) / 2} \lesssim 2^{j},
$$

which is (50).

Our next goal is to show that

$$
\int_{\mathbb{R}^{n} \backslash B^{*}}\left|K_{j}(x, y)\right| \mathrm{d} x \lesssim\left(2^{j} r\right)^{-1}, \quad y \in B, \quad r \geq 2^{-j} .
$$

To this end, define

$$
\widetilde{S}_{j, y}^{v, N} g_{j}^{\nu}(x):=2^{-j m^{\prime}} 2^{j n \rho} \int_{\Gamma_{j}^{\nu}} e^{i 2^{j \rho} x \cdot \xi}\left\{L^{N} \widetilde{b}_{j}^{v}\left(\mathbf{t}^{-1}(x+y), \xi\right)\right\} \widehat{g}_{j}^{v}(\xi) đ \xi,
$$

with

$$
\widetilde{b}_{j}^{\nu}(x, \xi):=a\left(x, 2^{j \rho} \xi\right) \chi_{j}^{\nu}(\xi) \psi_{j}\left(2^{j \rho} \xi\right) e^{i 2^{j \rho} h_{j}^{v}(x, \xi)}
$$


We observe that the same reasoning as in the proof of (52) reveals that for $N \geq 0$

$$
\left|L^{N} \widetilde{b}_{j}^{v}(x, \xi)\right| \lesssim 2^{j m}
$$

which in turn implies that $\widetilde{S}_{j, y}^{v, N}$ is a $\Psi$ DO of order $-n \max (0,(\delta-\rho) / 2)$ and hence once again $L^{2}$-bounded.

A similar calculation as in the case of $\nabla_{y} K_{j}(x, y)$ and estimate (28) yield that

$$
\begin{aligned}
& \left(\int_{\mathbb{R}^{n} \backslash B^{*}}\left|K_{j}^{v}(x, y)\right| \mathrm{d} x\right)^{2} \\
& \lesssim \int_{\mathbb{R}^{n} \backslash B^{*}} \frac{2^{2 j m^{\prime}}\left\|\tilde{S}_{j, y}^{\nu, N} g_{j}^{\nu}\right\|_{L^{2}\left(\mathbb{R}^{n}\right)}^{2}}{\left(1+2^{2 j \rho}\left|\left(\nabla \varphi\left(x, \xi_{j}^{\nu}\right)-y\right)_{1}\right|^{2}\right)^{2 N+2 / \rho-2 / \rho}} \\
& \times \frac{1}{\left(1+2^{j \rho}\left|\left(\nabla \varphi\left(x, \xi_{j}^{\nu}\right)-y\right)^{\prime}\right|^{2}\right)^{2 N+2 / \rho-2 / \rho}} \mathrm{d} x \\
& \lesssim \int_{\mathbb{R}^{n} \backslash B^{*}} \frac{2^{2 j m^{\prime}}\left\|\widetilde{S}_{j, y}^{\nu, N} g_{j}^{\nu}\right\|_{L^{2}\left(\mathbb{R}^{n}\right)}^{2}}{\left(2^{j \rho}\left|\left(\nabla \varphi\left(x, \xi_{j}^{\nu}\right)-y\right)_{1}\right|+2^{j \mu}\left|\left(\nabla \varphi\left(x, \xi_{j}^{\nu}\right)-y\right)^{\prime}\right|\right)^{4 / \rho}} \\
& \times \frac{1}{\left(1+2^{2 j \rho}\left|\left(\nabla \varphi\left(x, \xi_{j}^{\nu}\right)-y\right)_{1}\right|^{2}\right)^{2 N-2 / \rho}} \\
& \times \frac{1}{\left(1+2^{j \rho}\left|\left(\nabla \varphi\left(x, \xi_{j}^{\nu}\right)-y\right)^{\prime}\right|^{2}\right)^{2 N-2 / \rho}} \mathrm{d} x \\
& \lesssim \int_{\mathbb{R}^{n} \backslash B^{*}} \frac{2^{-2(j-k)} 2^{2 j m^{\prime}}\left\|\widetilde{S}_{j, y}^{\nu, N} g_{j}^{\nu}\right\|_{L^{2}\left(\mathbb{R}^{n}\right)}^{2}}{\left(1+2^{2 j \rho}\left|\left(\nabla \varphi\left(x, \xi_{j}^{\nu}\right)-y\right)_{1}\right|^{2}\right)^{2 N-2 / \rho}} \\
& \times \frac{1}{\left(1+2^{j \rho}\left|\left(\nabla \varphi\left(x, \xi_{j}^{v}\right)-y\right)^{\prime}\right|^{2}\right)^{2 N-2 / \rho}} \mathrm{d} x \\
& \lesssim 2^{-2(j-k)} 2^{-j(\rho+(n-1) \rho / 2)} 2^{2 j m^{\prime}}\left\|g_{j}^{\nu}\right\|_{L^{2}\left(\mathbb{R}^{n}\right)}^{2} \\
& \lesssim 2^{-2(j-k)} 2^{-j \rho(n+1) / 2)} 2^{2 j m^{\prime}} 2^{j(n+\rho(1-3 n) / 2)} \\
& =2^{-2(j-k)} 2^{j(1-2 n) \rho} \text {. }
\end{aligned}
$$

Hence,

$$
\int_{\mathbb{R}^{n} \backslash B^{*}}\left|K_{j}(x, y)\right| \mathrm{d} x \leq \sum_{\nu} 2^{-(j-k)} 2^{j(1-2 n) \rho / 2} \leq 2^{-(j-k)} \approx\left(2^{j} r\right)^{-1}
$$


Finally, given an atom $\mathfrak{a}$ with support in the ball $B:=B\left(x_{0}, r\right)$ with $r<1$ we write

$$
T_{a}^{\varphi} \mathfrak{a}(x)=\sum_{2^{j} \leq r^{-1}} T_{j} \mathfrak{a}(x)+\sum_{2^{j}>r^{-1}} T_{j} \mathfrak{a}(x),
$$

where as before, $r$ is the radius of the support of the atom a. Now observe that property (iii) of Definition 2.6 implies that

$$
\int_{B} K_{j}(x, \bar{y}) \mathfrak{a}(y) \mathrm{d} y=0,
$$

so this together with Minkowski's inequality, (51) and (53) yield that

$$
\begin{aligned}
\mathrm{II} & =\left\|T_{a}^{\varphi} \mathfrak{a}\right\|_{L^{1}\left(\mathbb{R}^{n} \backslash B^{*}\right)} \leq \sum_{2^{j} \leq r^{-1}}\left\|T_{j} \mathfrak{a}\right\|_{L^{1}\left(\mathbb{R}^{n} \backslash B^{*}\right)}+\sum_{2^{j}>r^{-1}}\left\|T_{j} \mathfrak{a}\right\|_{L^{1}\left(\mathbb{R}^{n} \backslash B^{*}\right)} \\
& \lesssim \sum_{2^{j} \leq r^{-1}} \int_{B}\left\|K_{j}(x, y)-K_{j}(x, \bar{y})\right\|_{L_{x}^{1}\left(\mathbb{R}^{n} \backslash B^{*}\right)}|\mathfrak{a}(y)| \mathrm{d} y \\
& +\sum_{2^{j}>r^{-1}} \int_{B}\left\|K_{j}(x, y)\right\|_{L_{x}^{1}\left(\mathbb{R}^{n} \backslash B^{*}\right)}|\mathfrak{a}(y)| \mathrm{d} y \\
& \lesssim \sum_{2^{j} \leq r^{-1}} \int_{B} 2^{j} r r^{-n} \mathrm{~d} y+\sum_{2^{j}>r^{-1}} \int_{B}\left(2^{j} r\right)^{-1} r^{-n} \mathrm{~d} y \\
\leq & \sum_{2^{j} \leq r^{-1}} 2^{j} r+\sum_{2^{j}>r^{-1}}\left(2^{j} r\right)^{-1} \lesssim 1 .
\end{aligned}
$$

The corresponding proof of the $\mathscr{H}^{1}-L^{1}$ boundedness of the adjoint $\left(T_{a}^{\varphi}\right)^{*}$ is similar to the one above with few modifications. First, regarding the $L^{2}$-boundedness in Lemma 6.3, estimate (46) in that Lemma has to be replaced by (47). Second, the $x$ and $y$ dependencies of the kernel are reversed. This means the following replacements:

$$
\begin{aligned}
& \nabla_{\xi} \varphi\left(x, \xi_{j}^{v}\right) \longrightarrow x, \\
& y \longrightarrow \nabla_{\xi} \varphi\left(y, \xi_{j}^{v}\right), \\
& \bar{y} \longrightarrow \nabla_{\xi} \varphi\left(\bar{y}, \xi_{j}^{v}\right) .
\end{aligned}
$$

Otherwise the proof remains the same.

Step 2 - Estimates of $\left\|\mathbf{T}_{\mathfrak{a}}^{\prime} \mathfrak{a}\right\|_{\mathbf{L}^{1}\left(\mathbb{R}^{\mathbf{n}}\right)}$ when $\mathbf{r} \geq \mathbf{1}$

Now we turn our attention to atoms with supports in balls of radii $r \geq 1$. In this case, using the compact support of the amplitude $a$ and the $L^{2}$-boundedness of $T_{a}^{\varphi}$ (Proposition 2.16) we have

$$
\left\|T_{a}^{\varphi} \mathfrak{a}\right\|_{L^{1}\left(\mathbb{R}^{n}\right)} \lesssim\left\|T_{a}^{\varphi} \mathfrak{a}\right\|_{L^{2}\left(\mathbb{R}^{n}\right)} \lesssim\|\mathfrak{a}\|_{L^{2}\left(\mathbb{R}^{n}\right)} \lesssim r^{-n / 2} \lesssim 1
$$


To prove the boundedness of the adjoint $\left(T_{a}^{\varphi}\right)^{*}$, we split the $L^{1}$-norm into two pieces, namely

$$
\int_{\mathbb{R}^{n}}\left|\left(T_{a}^{\varphi}\right)^{*} \mathfrak{a}(x)\right| \mathrm{d} x=\int_{B^{\prime}}\left|\left(T_{a}^{\varphi}\right)^{*} \mathfrak{a}(x)\right| \mathrm{d} x+\int_{\mathbb{R}^{n} \backslash B^{\prime}}\left|\left(T_{a}^{\varphi}\right)^{*} \mathfrak{a}(x)\right| \mathrm{d} x,
$$

where $B^{\prime}$ is the ball centered at the origin with radius $2 K$ and

$$
K:=\sup _{(y, \xi) \in \operatorname{supp} a \cap\left(\mathbb{R}^{n} \times \mathbb{S}^{n-1}\right)}\left|\nabla_{\xi} \varphi(y, \xi)\right| .
$$

We treat the first term of (55) as in (54). For the second term we observe that the kernel of $\left(T_{a}^{\varphi}\right)^{*}$ satisfies

$$
\left|\int_{\mathbb{R}^{n}} e^{i x \cdot \xi-i \varphi(y, \xi)} a(y, \xi) \mathrm{d} \xi\right| \lesssim \frac{1}{|x|^{N}},
$$

for $|x|>2 K$. This follows from the fact that, on the support of $a(y, \xi)$, the modulus of the gradient of the phase of the oscillatory integral above satisfies

$$
\left|x-\nabla_{\xi} \varphi(y, \xi)\right| \geq|x|-K \geq|x| / 2 .
$$

Now if

$$
\psi_{0}(\xi)+\sum_{j=1}^{\infty} \psi\left(2^{-j} \xi\right)=1
$$

is a Littlewood-Paley partition of unity with supp $\psi$ inside a fixed annulus (see Definition 2.1), then using Remark 6.1 (i.e. omitting the term where $j=0$ ) we have

$$
\begin{aligned}
\left|\int_{\mathbb{R}^{n}} e^{i x \cdot \xi-i \varphi(y, \xi)} a(y, \xi) \mathrm{d} \xi\right| & \lesssim \sum_{j=1}^{\infty}\left|\int_{\mathbb{R}^{n}} e^{i x \cdot \xi-i \varphi(y, \xi)} \psi\left(2^{-j} \xi\right) a(y, \xi) \mathrm{d} \xi\right| \\
& =\sum_{j=1}^{\infty} 2^{j n}\left|\int_{\mathbb{R}^{n}} e^{i \lambda \Phi(x, y, \xi)} b(y, \xi) \mathrm{d} \xi\right|
\end{aligned}
$$

with $\lambda:=2^{j}|x|$

$$
\Phi(x, y, \xi):=\frac{x \cdot \xi-\varphi(y, \xi)}{|x|}
$$

and

$$
b(y, \xi):=\psi(\xi) a\left(y, 2^{j} \xi\right)
$$

with compact support in $y$ and annulus-support in $\xi$. Now since for all multiindices $\alpha,\left|\partial_{\xi}^{\alpha} b(y, \xi)\right| \lesssim 2^{j m}$ and since for $(y, \xi) \in \operatorname{supp} b(y, \xi)$, (57) yields that 
$\left|\nabla_{\xi} \Phi(x, y, \xi)\right|=\frac{1}{|x|}\left|x-\nabla_{\xi} \varphi(y, \xi)\right| \gtrsim 1$, the non-stationary phase estimate of Lemma

2.18 could be used to deduce that

$$
\left|\int_{\mathbb{R}^{n}} e^{i \lambda \Phi(x, y, \xi)} b(y, \xi) đ \xi\right| \lesssim 2^{j m}\left(2^{j}|x|\right)^{-N},
$$

for any $N>0$. Thus using this in (58) and summing in $j,(56)$ follows. Hence

$$
\int_{\mathbb{R}^{n} \backslash B^{\prime}}\left|\left(T_{a}^{\varphi}\right)^{*} \mathfrak{a}(x)\right| \mathrm{d} x \lesssim \int_{\mathbb{R}^{n} \backslash B^{\prime}} \frac{1}{|x|^{N}}\left(\int_{B}|\mathfrak{a}(y)| \mathrm{d} y\right) \mathrm{d} x \lesssim 1 .
$$

\section{Step 3 - Globalisation of Steps 1 \& 2}

At this point we once again use the conditions on the phase function to reduce our analysis to the case of operators $T_{a}^{\varphi}$ with a phase function $\varphi(x, \xi)=\theta(x, \xi)+x \cdot \xi$, $\theta \in \Phi^{1}$ and $\theta(x, \xi)+x \cdot \xi$ is SND (see Sect. 3).

As far as the amplitude of $T_{a}^{\varphi}$ is concerned, we assume that $a(x, \xi)$ is as in the previous steps of the proof, albeit without the assumption of the compact support in $x$.

Our actual goal here is to globalise the results that we have obtained so far for both $T_{a}^{\varphi}$ and $\left(T_{a}^{\varphi}\right)^{*}$ at the same time. Whenever we write $T$ we refer to both $T_{a}^{\varphi}$ and $\left(T_{a}^{\varphi}\right)^{*}$.

Now with the reduction mentioned above we proceed to describe the globalisation procedure. In [22], Ruzhansky and Sugimoto developed a new technique to transfer local boundedness of Fourier integral operators, which was proven by Seeger, Sogge and Stein [23], to a global result, where the amplitudes of the corresponding operators do not have compact spatial supports.

Let us consider the FIO given by

$$
T f(x)=\iint_{\mathbb{R}^{2 n}} \sigma(x, y, \xi) e^{i(x-y) \cdot \xi+i \vartheta(x, y, \xi)} f(y) \mathrm{d} y \mathrm{~d} \xi,
$$

where $\sigma(x, y, \xi) \in \mathcal{C}^{\infty}\left(\mathbb{R}^{n} \times \mathbb{R}^{n} \times \mathbb{R}^{n}\right)$ satisfies the estimate

$$
\left|\partial_{\xi}^{\alpha} \partial_{x}^{\beta} \partial_{y}^{\gamma} \sigma(x, y, \xi)\right| \lesssim\langle\xi\rangle^{m-\rho|\alpha|+\delta|\beta+\gamma|},
$$

with $m \leq 0,0<\rho \leq 1,0 \leq \delta \leq 1$ for all multi-indices $\alpha, \beta$ and $\gamma$ and $(x, y, \xi) \in$ $\mathbb{R}^{n} \times \mathbb{R}^{n} \times \mathbb{R}^{n}$ (thus note that the case of $\rho=0$ is excluded due to a technical reason in the proof of Lemma 6.7 below). Here the phase function $\vartheta(x, y, \xi)$ is either $\theta(x, \xi)$ or $-\theta(y, \xi)$, with $\theta \in \Phi^{1}$ and is assumed to be smooth on the support of the amplitude $\sigma(x, y, \xi)$.

Then one defines the function

$$
H(x, y, z):=\inf _{\xi \in \mathbb{R}^{n}}\left|z+\nabla_{\xi} \vartheta(x, y, \xi)\right|
$$

and

$$
\Delta_{r}:=\left\{(x, y, z) \in \mathbb{R}^{n} \times \mathbb{R}^{n} \times \mathbb{R}^{n}: H(x, y, z) \geq r\right\} .
$$


We also define

$$
\widetilde{H}(z):=\inf _{x, y \in \mathbb{R}^{n}} H(x, y, z)=\inf _{x, y, \xi \in \mathbb{R}^{n}}\left|z+\nabla_{\xi} \vartheta(x, y, \xi)\right|
$$

and

$$
\widetilde{\Delta}_{r}:=\left\{z \in \mathbb{R}^{n}: \widetilde{H}(z) \geq r\right\}
$$

and set

$$
M_{L}:=\sum_{|\gamma| \leq L} \sup _{x, y, \xi \in \mathbb{R}^{n}}\left|\langle\xi\rangle^{-(m-\rho|\gamma|)} \partial_{\xi}^{\gamma} \sigma(x, y, \xi)\right|
$$

and

$$
N_{L}:=\sum_{1 \leq|\gamma| \leq L} \sup _{x, y, \xi \in \mathbb{R}^{n}}\left|\langle\xi\rangle^{-(1-|\gamma|)} \partial_{\xi}^{\gamma} \vartheta(x, y, \xi)\right| .
$$

Here we observe that $N_{L}<\infty$ by the $\Phi^{1}$-condition on $\theta$ above. Given these definitions one has the following lemma.

Lemma 6.6 Let $r \geq 1$ and $L \geq 1$. Then we have $\mathbb{R}^{n} \backslash \widetilde{\Delta}_{2 r} \subset\left\{z:|z|<\left(2+N_{L}\right) r\right\}$. Furthermore for $r>0, x \in \widetilde{\Delta}_{2 r}$ and $|y| \leq r$ we have

$$
\widetilde{H}(x) \leq 2 H(x, y, x-y)
$$

and therefore $(x, y, x-y) \in \Delta_{r}$.

Proof For $z \in \mathbb{R}^{n} \backslash \widetilde{\Delta}_{2 r}$, we have $\widetilde{H}(z)<2 r$. Hence, there exist $x_{0}, y_{0}, \xi_{0} \in \mathbb{R}^{n}$ such that

$$
\left|z+\nabla_{\xi} \vartheta\left(x_{0}, y_{0}, \xi_{0}\right)\right|<2 r .
$$

Since, $r \geq 1$, this yields that

$$
|z| \leq\left|z+\nabla_{\xi} \vartheta\left(x_{0}, y_{0}, \xi_{0}\right)\right|+\left|\nabla_{\xi} \vartheta\left(x_{0}, y_{0}, \xi_{0}\right)\right| \leq 2 r+N_{L} \leq\left(2+N_{L}\right) r .
$$

The claim that $(x, y, x-y) \in \Delta_{r}$ follows from (63) and the definition of $\Delta_{r}$. Therefore it only remains to prove (63). Now, if $|y| \leq r$ and $x \in \widetilde{\Delta}_{2 r}$ then since $\widetilde{H}(x) \geq 2 r$, we have that

$$
\begin{aligned}
\widetilde{H}(x) & \leq|x+\nabla \vartheta(x, y, \xi)| \leq|x-y+\nabla \vartheta(x, y, \xi)|+|y| \\
& \leq|x-y+\nabla \vartheta(x, y, \xi)|+\frac{\widetilde{H}(x)}{2} .
\end{aligned}
$$

From this, (63) follows at once. 
In order to prove the global boundedness, the following result is of particular importance.

Lemma 6.7 Let $\sigma(x, y, \xi)$ satisfy (60) with some $\rho \in(0,1]$. The kernel associated to the operator $T$ in (59) that is given by

$$
K(x, y, z):=\int_{\mathbb{R}^{n}} e^{i z \cdot \xi+i \vartheta(x, y, \xi)} \sigma(x, y, \xi) \mathrm{d} \xi
$$

is smooth on $\cup_{r>0} \Delta_{r}$. Moreover, for all $L>n / \rho$ and $r \geq 1$ it satisfies

$$
\left\|H^{L} K\right\|_{L^{\infty}\left(\Delta_{r}\right)} \leq C\left(L, M_{L}, N_{L+1}\right),
$$

where $C\left(L, M_{L}, N_{L+1}\right)$ is a positive constant depending only on $L, M_{L}$ and $N_{L+1}$. For $L>n$ and $r \geq 1$, the function $\widetilde{H}(z)$ satisfies the bound

$$
\left\|\widetilde{H}^{-L}\right\|_{L^{1}\left(\widetilde{\Delta}_{r}\right)} \leq C\left(L, N_{L+1}\right) .
$$

Proof If one introduces the differential operator

$$
D:=\frac{\left(z+\nabla_{\xi} \vartheta\right) \cdot \nabla_{\xi}}{i\left|z+\nabla_{\xi} \vartheta\right|^{2}}
$$

with the transpose $D^{*}$, then integrating by parts $L$ times yields

$$
K(x, y, z)=\int_{\mathbb{R}^{n}} e^{i z \cdot \xi+i \vartheta(x, y, \xi)}\left(D^{*}\right)^{L} \sigma(x, y, \xi) \mathrm{d} \xi .
$$

Now (65) follows from the relation

$$
r \leq H(x, y, z) \leq\left|z+\nabla_{\xi} \vartheta(x, y, \xi)\right|,
$$

which is valid for $(x, y, z) \in \Delta_{r}$ and $\xi \in \mathbb{R}^{n}$. Moreover

$$
|z| \leq\left|z+\nabla_{\xi} \vartheta(x, y, \xi)\right|+N_{L+1},
$$

for any $\xi \neq 0$, which yields that

$$
|z| \leq \widetilde{H}(z)+N_{L+1}
$$

Hence for $|z| \geq 2 N_{L+1}$ one has

$$
|z| \leq \widetilde{H}(z)+|z| / 2
$$

and therefore

$$
|z| \leq 2 \widetilde{H}(z)
$$


Using this we get

$$
\begin{aligned}
\left\|\widetilde{H}^{-L}\right\|_{L^{1}\left(\tilde{\Delta}_{r}\right)} & \leq\left\|\widetilde{H}^{-L}\right\|_{L^{1}\left(\tilde{\Delta}_{r} \cap\left\{|z| \leq 2 N_{L+1}\right\}\right)}+\left\|\widetilde{H}^{-L}\right\|_{L^{1}\left(\tilde{\Delta}_{r} \cap\left\{|z| \geq 2 N_{L+1}\right\}\right)} \\
& \leq r^{-L} \int_{|z| \leq 2 N_{L+1}} \mathrm{~d} z+2^{L} \int_{|z| \geq 2 N_{L+1}}|z|^{-L} \mathrm{~d} z \\
& \leq C\left(L, N_{L+1}\right),
\end{aligned}
$$

which proves (66) .

Now at this point we have all the tools that would help us to achieve our globalisation, and this amounts to prove that

$$
\int_{\mathbb{R}^{n}}|T \mathfrak{a}(x)| \mathrm{d} x \lesssim 1
$$

when there is no requirement on the support of the amplitude. To this end, first we observe that a global norm estimate for $T \mathfrak{a}$ with $\mathfrak{a}$ supported in a ball with an arbitrary centre, would follow from a norm-estimate that is uniform in $s$ for $\tau_{s}^{*} T \tau_{s} \mathfrak{a}$, with an atom $\mathfrak{a}$ whose support is inside a ball centred at the origin. Note that here $\tau_{s}$ is the operator of translation by $s \in \mathbb{R}^{n}$. This is because by translation invariance of the $L^{1}$-norm one has that

$$
\|T \mathfrak{a}\|_{L^{1}\left(\mathbb{R}^{n}\right)}=\left\|\tau_{s}^{*} T \tau_{s} \tau_{-s} \mathfrak{a}\right\|_{L^{1}\left(\mathbb{R}^{n}\right)} .
$$

Thus our goal is to establish that

$$
\left\|\tau_{S}^{*} T \tau_{s} \mathfrak{a}\right\|_{L^{1}\left(\mathbb{R}^{n}\right)} \lesssim 1
$$

where the estimate is uniform in $s$ and $\mathfrak{a}$ has its support in a ball centred at the origin.

Now let $r \geq 1, L>n / \rho$ and $s \in \mathbb{R}^{n}$ and suppose $\mathfrak{a}$ is an $\mathscr{H}^{1}$-atom supported in a ball $B$, centred at the origin, with radius $r$. We use the notions that were introduced in connection to the globalisation procedure in Section 3 and split the $L^{1}$-norm of $\tau_{s}^{*} T \tau_{s} \mathfrak{a}$ into the following two pieces:

$$
\left\|\tau_{s}^{*} T \tau_{s} \mathfrak{a}\right\|_{L^{1}\left(\mathbb{R}^{n}\right)}=\left\|\tau_{s}^{*} T \tau_{s} \mathfrak{a}\right\|_{L^{1}\left(\widetilde{\Delta}_{2 r}\right)}+\left\|\tau_{s}^{*} T \tau_{s} \mathfrak{a}\right\|_{L^{1}\left(\mathbb{R}^{n} \backslash \widetilde{\Delta}_{2 r}\right)},
$$

where $\widetilde{\Delta}_{r}$ is defined in (62). First let us show that

$$
\left\|\tau_{s}^{*} T \tau_{s} \mathfrak{a}\right\|_{L^{1}\left(\tilde{\Delta}_{2 r}\right)} \leq C\left(n, M_{L}, N_{L+1}\right) .
$$

By Lemma 6.6, for $x \in \widetilde{\Delta}_{2 r}$ and $|y| \leq r$, we have

$$
\widetilde{H}(x) \leq 2 H(x, y, x-y)
$$


and $(x, y, x-y) \in \Delta_{r}$. Now since for the kernel of the operator $T$ given by (64) one has that

$$
K(x, y, x-y)=\int_{\mathbb{R}^{n}} e^{i(x-y) \cdot \xi+i \vartheta(x, y, \xi)} \sigma(x, y, \xi) đ \xi
$$

(67) and Lemma 6.7 yield for any atom a supported in $B(0, r)$ that

$$
\begin{aligned}
|T \mathfrak{a}(x)| & \leq 2^{L} \widetilde{H}(x)^{-L} \int_{|y| \leq r}\left|H(x, y, x-y)^{L} K(x, y, x-y) \mathfrak{a}(y)\right| \mathrm{d} y \\
& \leq 2^{L} \widetilde{H}(x)^{-L}\left\|H^{L} K\right\|_{L^{\infty}\left(\Delta_{r}\right)}\|\mathfrak{a}\|_{L^{1}\left(\mathbb{R}^{n}\right)} \\
& \leq C\left(n, L, M_{L}, N_{L+1}\right) \widetilde{H}(x)^{-L}
\end{aligned}
$$

since $\|\mathfrak{a}\|_{L^{1}\left(\mathbb{R}^{n}\right)} \leq 1$. Therefore, if $r \geq 1$, choosing $L>n / \rho$, Lemma 6.7 and the monotonicity of $\Delta_{r}$ yield

$$
\|T \mathfrak{a}\|_{L^{1}\left(\widetilde{\Delta}_{2 r}\right)} \lesssim\left\|\widetilde{H}(x)^{-L}\right\|_{L^{1}\left(\widetilde{\Delta}_{2 r}\right)} \leq C\left(n, M_{L}, N_{L+1}\right) .
$$

Observe that the phase function and the amplitude of $\tau_{s}^{*} T \tau_{s}$ are of the form $\theta(x+$ $s, \xi)+(x-y) \cdot \xi$ and $\sigma(x+s, \xi)$ respectively when $T=T_{a}^{\varphi}$ (a similar property is also true for $\left.\left(T_{a}^{\varphi}\right)^{*}\right)$. Therefore the conjugation of $T$ by $\tau_{s}$ renders the constants $M_{L}$ and $N_{L+1}$ unchanged and therefore the estimate above also yields the very same one for $\tau_{s}^{*} T \tau_{s}$. This means that

$$
\left\|\tau_{s}^{*} T \tau_{s} \mathfrak{a}\right\|_{L^{1}\left(\widetilde{\Delta}_{2 r}\right)} \lesssim 1
$$

On the other hand for $\left\|\tau_{s}^{*} T \tau_{s} \mathfrak{a}\right\|_{L^{1}\left(\mathbb{R}^{n} \backslash \widetilde{\Delta}_{2 r}\right)}$, Lemma 6.6, Hölder's inequality and the properties of the atom $\mathfrak{a}$ yield that

$$
\begin{aligned}
\left\|\tau_{s}^{*} T \tau_{s} \mathfrak{a}\right\|_{L^{1}\left(\mathbb{R}^{n} \backslash \widetilde{\Delta}_{2 r}\right)} & \leq\left|\mathbb{R}^{n} \backslash \widetilde{\Delta}_{2 r}\right|^{1 / 2}\left\|\tau_{s}^{*} T \tau_{s} \mathfrak{a}\right\|_{L^{2}\left(\mathbb{R}^{n}\right)} \\
& \lesssim r^{n / 2}\|\mathfrak{a}\|_{L^{2}\left(\mathbb{R}^{n}\right)} \lesssim r^{n / 2} r^{-n / 2}=1 .
\end{aligned}
$$

Now if the atom is supported in a ball of radius $r \leq 1$ then clearly supp $\mathfrak{a} \subset B(0,1)$. Now write $\mathbb{R}^{n}=\widetilde{\Delta}_{2} \cup\left(\mathbb{R}^{n} \backslash \widetilde{\Delta}_{2}\right)$ and observe that we can now use Lemma 6.7 with $r=1$ to conclude that

$$
|T \mathfrak{a}(x)| \lesssim \widetilde{H}(x)^{-L},
$$

which in turn yields that

$$
\left\|\tau_{s}^{*} T \tau_{s} \mathfrak{a}\right\|_{L^{1}\left(\tilde{\Delta}_{2}\right)} \lesssim 1
$$

Finally, in view of the first part of Lemma 6.6 we see that $\mathbb{R}^{n} \backslash \widetilde{\Delta}_{2} \subset B\left(0,2+N_{L}\right)$ which together with the local boundedness result that we established in Steps 1 and 2 implies

$$
\left\|\tau_{S}^{*} T \tau_{s} \mathfrak{a}\right\|_{L^{1}\left(\mathbb{R}^{n} \backslash \widetilde{\Delta}_{2}\right)} \lesssim\left\|\tau_{s}^{*} T \tau_{s} \mathfrak{a}\right\|_{L^{1}\left(B\left(0,2+N_{L}\right)\right)} \lesssim\|\mathfrak{a}\|_{\mathscr{H}^{1}\left(\mathbb{R}^{n}\right)} \lesssim 1
$$


Now that we have boundedness from $\mathscr{H}^{1}\left(\mathbb{R}^{n}\right)$ to $L^{1}\left(\mathbb{R}^{n}\right)$ for both $T_{a}^{\varphi}$ itself and its adjoint, as well as $L^{2}$-boundedness, we can use a standard Riesz-Thorin interpolation argument to conclude that $T_{a}^{\varphi}$ is bounded from $L^{p}\left(\mathbb{R}^{n}\right)$ to itself.

\subsection{Forbidden amplitudes}

The case of operators with amplitudes in $S_{\rho, 1}^{m}\left(\mathbb{R}^{n}\right)$ with $0 \leq \rho \leq 1$ is rather special since the involved FIOs are, in general, not $L^{2}$-bounded. However, Proposition 2.16 yields that if $m<-n(1-\rho) / 2$ then the associated FIO is indeed $L^{2}$-bounded, and this result is sharp. Here, only for the sake of completeness of exposition we state the result proven in [11] regarding the $L^{p}$-boundedness of FIOs with forbidden amplitudes.

Proposition 6.8 Let $a \in S_{\rho, 1}^{m}\left(\mathbb{R}^{n}\right), \varphi$ be an SND phase function in the class $\Phi^{2}$ and let $T_{a}^{\varphi}$ be given as in Definition 2.15. For $0 \leq \rho \leq 1$ and

$$
m<-n(1-\rho) \max \left(\frac{1}{p}, \frac{1}{2}\right)-(n-1)\left|\frac{1}{p}-\frac{1}{2}\right|
$$

the FIO $T_{a}^{\varphi}$ is $L^{p}$-bounded for $1 \leq p \leq \infty$.

Proof See [17, Propositions 2.3 and 2.5] for the case $n=1$, which is essentially the pseudodifferential case, and [11, Theorem 2.17] for $n \geq 2$.

\section{Sobolev space boundedness of FIOs with $S_{1,1}^{0}$-amplitudes}

It turns out that just as in the case of pseudodifferential operators, the FIOs with forbidden amplitudes, say in $S_{1,1}^{0}\left(\mathbb{R}^{n}\right)$, despite failing to be $L^{2}$-bounded are bounded on $H^{s}\left(\mathbb{R}^{n}\right)$ with $s>0$. As it was mentioned in the introduction, the proof of the Sobolevboundedness in the pseudodifferential case goes back to E. Stein and independently to Y. Meyer. Other proofs were given by Bourdaud [3] and Hörmander [15]. Following Bourdaud, we establish the Sobolev boundedness of FIOs with amplitudes in the class $S_{1,1}^{0}\left(\mathbb{R}^{n}\right)$.

Proposition 7.1 Let $r>0, a \in C_{*}^{r} S_{1,1}^{0}\left(\mathbb{R}^{n}\right)$ and $\varphi$ be an SND phase function in the class $\Phi^{2}$. Then for $\max (0, r-1 / 2)<s<r$, the FIO $T_{a}^{\varphi}$ is bounded from the Sobolev space $H^{s}\left(\mathbb{R}^{n}\right)$ to $H^{s}\left(\mathbb{R}^{n}\right)$.

Proof We divide the proof into five steps.

Step 1 - Reduction of the FIOs with amplitudes in $\mathbf{C}_{*}^{\mathbf{r}} \mathbf{S}_{1,1}^{\mathbf{0}}\left(\mathbb{R}^{\mathbf{n}}\right)$ class

Following [3], for an amplitude in $C_{*}^{r} S_{1,1}^{0}\left(\mathbb{R}^{n}\right)$ one has the decomposition

$$
a(x, \xi)=\sum_{k=0}^{\infty} M_{k}(x) \psi_{k}(\xi)
$$


where $\psi_{k}$ was introduced in Definition 2.1 and $M_{k}(x)$ satisfies

$$
\left|M_{k}(x)\right| \lesssim 1, \quad\left\|M_{k}\right\|_{C_{*}^{r}\left(\mathbb{R}^{n}\right)} \lesssim 2^{k r}
$$

The $C_{*}^{r}$-norm is given in Definition 2.9.

We treat the case $k=0$ (the low frequency portion of the FIO) separately, so for now assume that $k \geq 1$.

Using the Littlewood-Paley partition of unity $I=\sum_{j=0}^{\infty} \psi_{j}(D)$ and setting

$$
M_{k j}(x):=\psi_{j}(D) M_{k}(x), \quad f_{k}:=\psi_{k}(D) f \quad \text { and } \quad F_{k}:=T_{1}^{\varphi} f_{k},
$$

(the amplitude of the FIO $T_{1}^{\varphi}$ is identically equal to one) we have that for $k \geq 1$ and $j \geq 0$

$$
\left\|M_{k j}\right\|_{L^{\infty}\left(\mathbb{R}^{n}\right)} \lesssim 2^{r(k-j)} \quad \text { and } \quad\left\|F_{k}\right\|_{L^{2}\left(\mathbb{R}^{n}\right)} \lesssim\left\|f_{k}\right\|_{L^{2}\left(\mathbb{R}^{n}\right)} .
$$

Denoting the inverse Fourier transform of a function $\psi$ by $\psi^{\vee}$, the first estimate in (71) can be shown using the bound $\left\|M_{k}\right\|_{C_{*}^{r}\left(\mathbb{R}^{n}\right)} \lesssim 2^{k r}$, the properties of the Zygmund class given in (3) and that

$$
\int_{\mathbb{R}^{n}} 2^{j n} \psi^{\vee}\left(\frac{y}{2^{-j}}\right) \mathrm{d} y=\int_{\mathbb{R}^{n}} \psi^{\vee}(y) \mathrm{d} y=0,
$$

as follows

$$
\begin{aligned}
\left|\psi_{j}(D)\left(M_{k}\right)(x)\right| & \lesssim\left|\int_{\mathbb{R}^{n}} 2^{j n} \psi^{\vee}\left(\frac{y}{2^{-j}}\right)\left(M_{k}(x-y)-M_{k}(x)\right) \mathrm{d} y\right| \\
& \lesssim 2^{-j r+k r} \int_{\mathbb{R}^{n}}\left|2^{j n} \psi^{\vee}\left(\frac{y}{2^{-j}}\right)\right| \frac{|y|^{r}}{2^{-j r}} \mathrm{~d} y \lesssim 2^{(k-j) r},
\end{aligned}
$$

for $j>0$. For $j=0$ this is a consequence of the $L^{\infty}$-boundedness of $\psi_{0}(D)$ and the first estimate in (70).

The second estimate in (71) is of course a direct consequence of the $L^{2}$-boundedness of FIOs with amplitudes in $S_{1,0}^{0}\left(\mathbb{R}^{n}\right)$.

Using the above notation we can now decompose $T_{a}^{\varphi}$ as

$$
T_{a}^{\varphi} f(x)=\sum_{k=1}^{\infty} M_{k}(x) T_{1}^{\varphi} f_{k}(x)=\sum_{k=1}^{\infty} M_{k}(x) F_{k}(x) .
$$

At this point, taking into account the properties of the SND phase function $\varphi \in \Phi^{2}$ (i.e. $\left.\left|\nabla_{x} \varphi(x, \xi)\right| \approx|\xi|\right)$, Proposition 2.19 and choosing a smooth annulus-supported $\tilde{\psi}$ with $\psi \cdot\left(\tilde{\psi} \circ \nabla_{x} \varphi(x, \cdot)\right)=\psi$, we have for any integer $N_{1}>0$ and any $0<\varepsilon<1 / 2$

$$
F_{k}(x)=\int_{\mathbb{R}^{n}} \tilde{\psi}_{k}\left(\nabla_{x} \varphi(x, \xi)\right) e^{i \varphi(x, \xi)} \widehat{f}_{k}(\xi) đ \xi
$$




$$
\begin{aligned}
& =\tilde{\psi}_{k}(D) F_{k}(x)-\sum_{0<|\alpha|<N_{1}} \frac{2^{-k \varepsilon|\alpha|}}{\alpha !} T_{\sigma_{\alpha, k}}^{\varphi} f_{k}(x)-2^{-k \varepsilon N_{1}} T_{r_{k}}^{\varphi} f_{k}(x) \\
& =: F_{k}^{1}(x)+F_{k}^{2}(x)+F_{k}^{3}(x),
\end{aligned}
$$

with

$$
\begin{gathered}
\left|\partial_{\xi}^{\beta} \partial_{x}^{\gamma} \sigma_{\alpha, k}(x, \xi)\right| \lesssim\langle\xi\rangle^{-(1 / 2-\varepsilon)|\alpha|-|\beta|}, \quad|\alpha| \geq 0, \\
\operatorname{supp}_{\xi} \sigma_{\alpha, k}(x, \xi)=\left\{\xi \in \mathbb{R}^{n}: C_{1} 2^{k} \leq|\xi| \leq C_{2} 2^{k}\right\},
\end{gathered}
$$

and

$$
\left|\partial_{\xi}^{\beta} \partial_{x}^{\gamma} r_{k}(x, \xi)\right| \lesssim\langle\xi\rangle^{-(1 / 2-\varepsilon) N_{1}-|\beta|},
$$

where the estimates above are uniform in $k$.

Thus

$$
T_{a}^{\varphi} f(x)=\sum_{k=1}^{\infty} M_{k}(x) F_{k}^{1}(x)+\sum_{k=1}^{\infty} M_{k}(x) F_{k}^{2}(x)+\sum_{k=1}^{\infty} M_{k}(x) F_{k}^{3}(x) .
$$

Step 2 - Analysis of $\sum_{k=1}^{\infty} M_{k}(x) F_{k}^{1}(x)$

Now to analyse $F_{k}^{1}$ we write

$$
M_{k}=\sum_{j=0}^{\infty} \psi_{j}(D) M_{k}=: \sum_{j=0}^{\infty} M_{k j}
$$

and split the sum in $j$ into the following pieces

$$
\begin{aligned}
\sum_{k=1}^{\infty} \sum_{j=0}^{\infty} M_{k j}(x) F_{k}^{1}(x) & =\sum_{k=1}^{\infty} \sum_{j=0}^{k-1} M_{k j}(x) F_{k}^{1}(x)+\sum_{k=1}^{\infty} \sum_{j=k}^{\infty} M_{k j}(x) F_{k}^{1}(x) \\
& =: \mathbf{A}+\mathbf{B} .
\end{aligned}
$$

Firstly, we establish the $H^{s}$-boundedness of $\mathbf{A}$. To this end we have

$$
\sum_{k=1}^{\infty} \sum_{j=0}^{k-1} M_{k j}(x) F_{k}^{1}(x)=: \sum_{k=1}^{\infty} b_{k}(x)
$$

The Fourier transform of $b_{k}$ is given by

$$
\widehat{b_{k}}(\eta)=\sum_{j=0}^{k-1} \int_{\mathbb{R}^{n}} \psi_{j}(\eta-\xi) \widehat{M}_{k}(\eta-\xi) \tilde{\psi}_{k}(\xi) \widehat{F_{k}}(\xi) \mathrm{d} \xi
$$


By recalling the defining property (2) of the Littlewood-Paley decomposition and the telescoping property of their partial sums, we see that the Fourier transform of $b_{k}$ satisfies

$$
\begin{aligned}
\widehat{b_{k}}(\eta) & =\sum_{j=0}^{k-1} \int \psi_{j}(\eta-\xi) \widehat{M}_{k}(\eta-\xi) \tilde{\psi}_{k}(\xi) \widehat{F}_{k}(\xi) đ \xi \\
& =\int \psi_{k-1}(\eta-\xi) \widehat{M}_{k}(\eta-\xi) \tilde{\psi}_{k}(\xi) \widehat{F}_{k}(\xi) đ \xi \\
& =\int \psi_{0}\left(2^{-(k-2)}(\eta-\xi)\right) \widehat{M}_{k}(\eta-\xi) \tilde{\psi}_{k}(\xi) \widehat{F}_{k}(\xi) đ \xi
\end{aligned}
$$

Note using the information in Definition 2.1 regarding the support of $\psi_{0}$ we see that $|\eta-\xi| \leq 2^{k-1}$, and due to the fact that $\tilde{\psi}$ is supported in an annulus, we have $|\xi| \sim 2^{k}$. Therefore the support of $\widehat{b_{k}}$ is bounded above by $|\eta| \leq|\eta-\xi|+|\xi| \lesssim 2^{k}\left(2^{-1}+1\right)$, and from below by $|\eta| \geq|\xi|-|\eta-\xi| \gtrsim 2^{k}\left(1-2^{-1}\right)$. From this it follows that the spectrum of $b_{k}$ is contained in an annulus $|\eta| \approx 2^{k}$. Hence by Lemma 2.5 it follows that for $s>0$,

$$
\begin{aligned}
\left\|\sum_{k=1}^{\infty} b_{k}\right\|_{H^{s}\left(\mathbb{R}^{n}\right)} & \lesssim\left\|\left\{\sum_{k=1}^{\infty} 4^{k s}\left|\sum_{j=0}^{k-1} M_{k j} F_{k}^{1}\right|^{2}\right\}^{1 / 2}\right\|_{L^{2}\left(\mathbb{R}^{n}\right)} \\
& \lesssim\left\|\left\{\sum_{k=1}^{\infty} 4^{k s}\left\|M_{k}\right\|_{L^{\infty}\left(\mathbb{R}^{n}\right)}^{2}\left|F_{k}^{1}\right|^{2}\right\}^{1 / 2}\right\|_{L^{2}\left(\mathbb{R}^{n}\right)} \\
& \lesssim\left\|\left\{\sum_{k=1}^{\infty} 4^{k s}\left|F_{k}^{1}\right|^{2}\right\}^{1 / 2}\right\|{ }_{L^{2}\left(\mathbb{R}^{n}\right)}=\left\{\sum_{k=1}^{\infty} 4^{k s}\left\|F_{k}^{1}\right\|_{L^{2}\left(\mathbb{R}^{n}\right)}^{2}\right\}^{1 / 2} \\
& \lesssim\left\{\sum_{k=1}^{\infty} 4^{k s}\left\|f_{k}\right\|_{L^{2}\left(\mathbb{R}^{n}\right)}^{2}\right\}^{1 / 2} \lesssim\|f\|_{H^{s}\left(\mathbb{R}^{n}\right)}
\end{aligned}
$$

where we have used Remark 2.4 and that

$$
\left|\sum_{j=0}^{k-1} M_{k j}\right| \lesssim\left\|M_{k}\right\|_{L^{\infty}\left(\mathbb{R}^{n}\right)} \lesssim 1
$$

Now for term $\mathbf{B}$ in (73), applying Lemma 2.5 to

$$
h_{j}(x):=\sum_{k=1}^{j} M_{k j}(x) F_{k}^{1}(x),
$$

we obtain (using Fubini's theorem for sums, (71) and Young's inequality for discrete convolutions) 


$$
\begin{aligned}
\left\|\sum_{k=1}^{\infty} \sum_{j=k}^{\infty} M_{k j} F_{k}^{1}\right\|_{H^{s}\left(\mathbb{R}^{n}\right)} \lesssim\left\|\sum_{j=1}^{\infty} \sum_{k=1}^{j} M_{k j} F_{k}^{1}\right\|_{H^{s}\left(\mathbb{R}^{n}\right)} \\
=\left\|\sum_{j=1}^{\infty} h_{j}\right\|_{H^{s}\left(\mathbb{R}^{n}\right)} \lesssim\left\|\left\{\sum_{j=1}^{\infty} 4^{j s}\left|h_{j}\right|^{2}\right\}^{1 / 2}\right\|_{L^{2}\left(\mathbb{R}^{n}\right)} \\
\quad \lesssim\left\|\left\{\sum_{j=1}^{\infty} 4^{j s}\left(\sum_{k=1}^{j} 2^{(k-j) r}\left|F_{k}^{1}\right|\right)^{2}\right\}^{1 / 2}\right\|_{L^{2}\left(\mathbb{R}^{n}\right)} \\
=\left\|\left\{\sum_{j=0}^{\infty}\left(2^{(\cdot)(s-r)} * 2^{(\cdot) s}\left|F_{(\cdot)}^{1}(j)\right|\right)^{2}\right\}^{1 / 2}\right\|_{L^{2}\left(\mathbb{R}^{n}\right)} \\
\quad \lesssim\left\|\left\{\sum_{j=0}^{\infty} 2^{j(s-r)}\right\}\left\{\sum_{0}^{\infty}\left(2^{j s} \tilde{\psi}_{j}(D) F_{j}\right)^{2}\right\}^{1 / 2}\right\|_{L^{2}\left(\mathbb{R}^{n}\right)} \\
\quad \lesssim\|f\|_{H^{s}\left(\mathbb{R}^{n}\right)},
\end{aligned}
$$

because of Remarks 2.3, 2.4 and $r>s$.

Step 3 - Analysis of $\sum_{k=1}^{\infty} M_{k}(x) F_{k}^{2}(x)$

To establish the Sobolev boundedness for the term $\sum_{k=1}^{\infty} M_{k}(x) F_{k}^{2}(x)$, we need to analyse the action of the Littlewood-Paley operator $\psi_{j}(D)$ on this term in order to use Definition 2.2 together with Remark 2.4. Here we also assume that $r \in(s, s+\varepsilon)$, where $\varepsilon \in(0,1 / 2)$ is given in representation of $F_{k}(x)$ in (72).

Then, for an integer $N_{2}>0$ and $0<\varepsilon^{\prime}<1 / 2$, write

$$
\begin{aligned}
\psi_{j}(D)\left(M_{k}(x) F_{k}^{2}\right)= & M_{k}(x) \psi_{j}(D) F_{k}^{2}(x)+\left[\psi_{j}(D), M_{k}\right] F_{k}^{2}(x) \\
= & M_{k}(x) \sum_{0<|\alpha|<N_{1}} \frac{2^{-k \varepsilon|\alpha|}}{\alpha !}\left(\sum_{|\beta|<N_{2}} \frac{2^{-j \varepsilon^{\prime}|\beta|}}{\beta !} T_{\sigma_{\alpha, \beta, k, j}}^{\varphi}\right. \\
& \left.+2^{-j \varepsilon^{\prime} N_{2}} T_{r_{j, k}}^{\varphi}\right) f_{k}(x) \\
& +\left[\psi_{j}(D), M_{k}\right] F_{k}^{2} \\
= & : \mathrm{I}+\mathrm{II}+\mathrm{III},
\end{aligned}
$$

with

$$
\begin{gathered}
\left|\partial_{\xi}^{\delta} \partial_{x}^{\gamma} \sigma_{\alpha, \beta, k, j}(x, \xi)\right| \lesssim\langle\xi\rangle^{-(1 / 2-\varepsilon)|\alpha|-\left(1 / 2-\varepsilon^{\prime}\right)|\beta|-|\delta|}, \quad|\alpha|>0, \quad|\beta| \geq 0, \\
\operatorname{supp}_{\xi} \sigma_{\alpha, \beta, k, j}(x, \xi)=\left\{\xi \in \mathbb{R}^{n}: C_{1} 2^{j} \leq|\xi| \leq C_{2} 2^{j}\right\}
\end{gathered}
$$

and

$$
\left|\partial_{\xi}^{\delta} \partial_{x}^{\gamma} r_{j, k}(x, \xi)\right| \lesssim\langle\xi\rangle^{-(1 / 2-\varepsilon) N_{1}-\left(1 / 2-\varepsilon^{\prime}\right) N_{2}-|\delta|},
$$


where both estimates above are uniform in $j$ and $k$. Moreover, since in the decomposition (69) of $a(x, \xi)$, we are at present considering the parts supported outside a neighbourhood of the origin in the $\xi$-variable, i.e. those for which $k \geq 1$, we also have that $r_{j, k}(x, \xi)$ vanishes in a neighbourhood of $\xi=0$.

For term I, and in view of the support properties of $\sigma_{\alpha, \beta, k, j}$, we claim that (uniformly in $j$ and $k$ )

$$
\left\|T_{\sigma_{\alpha, \beta, k, j}}^{\varphi} f\right\|_{L^{2}\left(\mathbb{R}^{n}\right)} \lesssim\left\|\Psi_{j}(D) f\right\|_{L^{2}\left(\mathbb{R}^{n}\right)},
$$

where $\Psi_{j}$ is a Littlewood-Paley-type frequency localisation that is equal to one on the support of $\sigma_{\alpha, \beta, k, j}$. Therefore $T_{\sigma_{\alpha, \beta, k, j}}^{\varphi} f=T_{\sigma_{\alpha, \beta, k, j}}^{\varphi} \Psi_{j}(D) f$, and it is enough to show that

$$
\left\|T_{\sigma_{\alpha, \beta, k, j}}^{\varphi} f\right\|_{L^{2}\left(\mathbb{R}^{n}\right)} \lesssim\|f\|_{L^{2}\left(\mathbb{R}^{n}\right)},
$$

uniformly in $k$ and $j$. To see this, we proceed by studying the boundedness of $S_{j}:=$ $T_{\sigma_{\alpha, \beta, k, j}}^{\varphi}\left(T_{\sigma_{\alpha, \beta, k, j}}^{\varphi}\right)^{*}$. A simple calculation shows that

$$
S_{j} f(x)=\int_{\mathbb{R}^{n}} K_{j}(x, y) f(y) \mathrm{d} y,
$$

with

$$
K_{j}(x, y):=\int_{\mathbb{R}^{n}} e^{i \varphi(x, \xi)-i \varphi(y, \xi)} \sigma_{\alpha, \beta, k, j}(x, \xi) \overline{\sigma_{\alpha, \beta, k, j}(y, \xi)} \mathrm{d} \xi .
$$

Now, since $\varphi$ is homogeneous of degree one in the $\xi$ variable, $K_{j}(x, y)$ can be written as

$$
K_{j}(x, y)=2^{j n} \int_{\mathbb{R}^{n}} b_{j}\left(x, y, 2^{j} \xi\right) e^{i 2^{j} \Phi(x, y, \xi)} \mathrm{d} \xi,
$$

with

$$
\Phi(x, y, \xi):=\varphi(x, \xi)-\varphi(y, \xi)
$$

and

$$
b_{j}(x, y, \xi):=\sigma_{\alpha, \beta, k, j}(x, \xi) \overline{\sigma_{\alpha, \beta, k, j}(y, \xi)} .
$$

Observe that the $\xi$-support of $b_{j}\left(x, y, 2^{j} \xi\right)$ lies in the compact set $\mathcal{K}:=\left\{C_{1} \leq|\xi|\right.$ $\leq C_{2}$ \}. From the SND condition (5) it also follows that

$$
\left|\nabla_{\xi} \Phi(x, y, \xi)\right| \approx|x-y|, \quad \text { for any } x, y \in \mathbb{R}^{n} \text { and } \xi \in \mathcal{K}
$$

Assume that $N_{3}>n$ is an integer, fix $x \neq y$ and $\operatorname{set} \phi(\xi):=\Phi(x, y, \xi), \vartheta:=\left|\nabla_{\xi} \phi\right|^{2}$. By the mean value theorem, (4) and (77), for any multi-index $\alpha$ with $|\alpha| \geq 1$ and any $\xi \in \mathcal{K}$,

$$
\left|\partial_{\xi}^{\alpha} \phi(\xi)\right| \lesssim\left|\nabla_{\xi} \Phi(x, y, \xi)\right|=\vartheta^{1 / 2}
$$


On the other hand, since

$$
\partial_{\xi}^{\alpha} \vartheta=\sum_{\nu=1}^{n} \sum_{\beta \leq \alpha}\left(\begin{array}{l}
\alpha \\
\beta
\end{array}\right) \partial_{\xi}^{\beta} \partial_{\xi_{\nu}} \phi \partial_{\xi}^{\alpha-\beta} \partial_{\xi_{\nu}} \phi
$$

it follows that, for any $|\alpha| \geq 0,\left|\partial_{\xi}^{\alpha} \vartheta\right| \lesssim \vartheta$. We estimate the kernel $K_{j}$ in two different ways. For the first estimate, (77) and Lemma 2.18 with $F(\xi):=b_{j}\left(x, y, 2^{j} \xi\right)$, yield

$$
\begin{aligned}
& \left|K_{j}(x, y)\right| \\
& \quad \leq 2^{j n} 2^{-j N_{3}} C_{M, \mathcal{K}} \sum_{|\alpha| \leq N_{3}} 2^{j|\alpha|} \int_{\mathbb{R}^{n}}\left|\partial_{\xi}^{\alpha} b_{j}\left(x, y, 2^{j} \xi\right)\right|\left|\nabla_{\xi} \Phi(x, y, \xi)\right|^{-N_{3}} \mathrm{~d} \xi \\
& \quad \lesssim 2^{-j N_{3}}|x-y|^{-N_{3}} \sum_{|\alpha| \leq N_{3}} 2^{j|\alpha|} \int_{\mathbb{R}^{n}}\left|\partial_{\xi}^{\alpha} b_{j}(x, y, \xi)\right| \mathrm{d} \xi \\
& \lesssim 2^{j n}\left(2^{j}|x-y|\right)^{-N_{3}}
\end{aligned}
$$

where the fact that the $\xi$-support of $b_{j}$ lies in a ball of radius $\approx 2^{j}$ and that for $|\alpha| \geq 0$

$$
\left|\partial_{\xi}^{\alpha} b_{j}(x, y, \xi)\right| \lesssim 2^{-j|\alpha|}
$$

have been used. By (79) we also obtain

$$
\left|K_{j}(x, y)\right| \leq 2^{j n} \int_{\mathbb{R}^{n}}\left|b_{j}\left(x, y, 2^{j} \xi\right)\right| đ \xi \lesssim 2^{j n},
$$

and when combining estimates (78) and (80) one has

$$
\left|K_{j}(x, y)\right| \lesssim 2^{j n}\left(1+2^{j}|x-y|\right)^{-N_{3}} .
$$

Thus, using (81) and Minkowski's inequality we have

$$
\left\|S_{j} f\right\|_{L^{2}\left(\mathbb{R}^{n}\right)} \lesssim\left\|\int_{\mathbb{R}^{n}} 2^{j n}\left(1+2^{j}|y|\right)^{-N_{3}} f(\cdot-y) \mathrm{d} y\right\|_{L^{2}\left(\mathbb{R}^{n}\right)} \lesssim\|f\|_{L^{2}\left(\mathbb{R}^{n}\right)} .
$$

The Cauchy-Schwarz inequality yields

$$
\begin{aligned}
\left\|\left(T_{\sigma_{\alpha, \beta, k, j}}^{\varphi}\right)^{*} f\right\|_{L^{2}\left(\mathbb{R}^{n}\right)}^{2} & =\left\langle T_{\sigma_{\alpha, \beta, k, j}}^{\varphi}\left(T_{\sigma_{\alpha, \beta, k, j}}^{\varphi}\right)^{*} f, f\right\rangle_{L^{2}\left(\mathbb{R}^{n}\right)} \\
& \lesssim\left\|S_{j} f\right\|_{L^{2}\left(\mathbb{R}^{n}\right)}\|f\|_{L^{2}\left(\mathbb{R}^{n}\right)} \\
& \lesssim\|f\|_{L^{2}\left(\mathbb{R}^{n}\right)}^{2}
\end{aligned}
$$


Therefore

$$
\left\|T_{\sigma_{\alpha, \beta, k, j}}^{\varphi}\right\|_{L^{2}\left(\mathbb{R}^{n}\right) \rightarrow L^{2}\left(\mathbb{R}^{n}\right)}=\left\|\left(T_{\sigma_{\alpha, \beta, k, j}}^{\varphi}\right)^{*}\right\|_{L^{2}\left(\mathbb{R}^{n}\right) \rightarrow L^{2}\left(\mathbb{R}^{n}\right)} \lesssim 1
$$

and (76) is proven.

Observe that (70), Cauchy-Schwarz inequality, Fubini's theorem, (75), and the definition of the Sobolev norm yield

$$
\begin{aligned}
& \sum_{j=0}^{\infty} 4^{j s}\left\|\sum_{k=1}^{\infty} M_{k}(x) \sum_{0<|\alpha|<N_{1}} \frac{2^{-k \varepsilon|\alpha|}}{\alpha !} \sum_{|\beta|<N_{2}} \frac{2^{-j \varepsilon^{\prime}|\beta|}}{\beta !} T_{\sigma_{\alpha, \beta, k, j}}^{\varphi} f_{k}\right\|_{L^{2}\left(\mathbb{R}^{n}\right)}^{2} \\
& \quad \lesssim \sum_{k=1}^{\infty} 2^{-k \varepsilon} \sum_{j=0}^{\infty} 4^{j s}\left\|\Psi_{j}(D) f_{k}\right\|_{L^{2}\left(\mathbb{R}^{n}\right)}^{2} \lesssim \sum_{k=1}^{\infty} 2^{-k \varepsilon}\left\|f_{k}\right\|_{H^{s}\left(\mathbb{R}^{n}\right)}^{2} \lesssim\|f\|_{H^{s}\left(\mathbb{R}^{n}\right)}^{2}
\end{aligned}
$$

For term II, we decompose $T_{r_{j, k}}^{\varphi}$ into Littlewood-Paley pieces as follows:

$$
T_{r_{j, k}}^{\varphi} f(x)=\sum_{\ell=0}^{\infty} \int_{\mathbb{R}^{n}} e^{i \varphi(x, \xi)} r_{j, k}(x, \xi) \psi_{\ell}(\xi) \widehat{f}(\xi) \mathrm{d} \xi=: \sum_{\ell=0}^{\infty} T_{r_{j, k}, \ell}^{\varphi} f(x)
$$

where the $\psi_{\ell}$ 's are defined in Definition 2.1. By a proof identical to the one of (75), we see that

$$
\left\|T_{r_{j, k, \ell}}^{\varphi} f\right\|_{L^{2}\left(\mathbb{R}^{n}\right)} \lesssim\left\|\Psi_{\ell}(D) f\right\|_{L^{2}\left(\mathbb{R}^{n}\right)}
$$

Thus

$$
\left\|T_{r_{j, k}}^{\varphi} f\right\|_{L^{2}\left(\mathbb{R}^{n}\right)} \lesssim \sum_{\ell=0}^{\infty}\left\|T_{r_{j, k, \ell}}^{\varphi} f\right\|_{L^{2}\left(\mathbb{R}^{n}\right)} \lesssim \sum_{\ell=0}^{\infty}\left\|\Psi_{\ell}(D) f\right\|_{L^{2}\left(\mathbb{R}^{n}\right)}
$$

Note that the estimate (83) is uniform in $j$. Then we claim that for $s>0$ one has

$$
T_{r_{j, k}}^{\varphi}: H^{s}\left(\mathbb{R}^{n}\right) \rightarrow L^{2}\left(\mathbb{R}^{n}\right)
$$

Indeed the Cauchy-Schwarz inequality yields

$$
\begin{aligned}
\left\|T_{r_{j, k}}^{\varphi} f\right\|_{L^{2}\left(\mathbb{R}^{n}\right)} & \lesssim \sum_{\ell=0}^{\infty}\left\|\Psi_{\ell}(D) f\right\|_{L^{2}\left(\mathbb{R}^{n}\right)}=\sum_{\ell=0}^{\infty} 2^{-\ell s}\left(2^{\ell s}\left\|\Psi_{\ell}(D) f\right\|_{L^{2}\left(\mathbb{R}^{n}\right)}\right) \\
& \lesssim\left(\sum_{\ell=0}^{\infty} 2^{-2 \ell s}\right)^{1 / 2}\left(\sum_{\ell=0}^{\infty} 2^{2 \ell s}\left\|\Psi_{\ell}(D) f\right\|_{L^{2}\left(\mathbb{R}^{n}\right)}^{2}\right)^{1 / 2} \lesssim\|f\|_{H^{s}\left(\mathbb{R}^{n}\right)}
\end{aligned}
$$


The last step follows from the definition of $H^{s}\left(\mathbb{R}^{n}\right)$, see Remark 2.3. Thus, for $N_{2}>\frac{s}{\varepsilon^{\prime}}$,

$$
\begin{aligned}
\sum_{j=0}^{\infty} 4^{j s} & \left\|\sum_{k=1}^{\infty} M_{k} 2^{-j \varepsilon^{\prime} N_{2}} \sum_{0<|\alpha|<N_{1}} \frac{2^{-k \varepsilon|\alpha|}}{\alpha !} T_{r_{j, k}}^{\varphi} f_{k}\right\|_{L^{2}\left(\mathbb{R}^{n}\right)}^{2} \\
& \lesssim\left(\sum_{k=1}^{\infty} 2^{-k \varepsilon}\right)^{2} \sum_{j=0}^{\infty} 4^{j\left(s-\varepsilon^{\prime} N_{2}\right)}\|f\|_{H^{s}\left(\mathbb{R}^{n}\right)}^{2} \lesssim\|f\|_{H^{s}\left(\mathbb{R}^{n}\right)}^{2}
\end{aligned}
$$

For term III of (74), the second estimate in (70) and relations (3) yield that

$$
\begin{aligned}
\left|\left[\psi_{j}(D), M_{k}\right]\left(F_{k}^{2}\right)(x)\right| & \lesssim\left|\int_{\mathbb{R}^{n}} 2^{j n} \psi^{\vee}\left(\frac{x-y}{2^{-j}}\right)\left(M_{k}(y)-M_{k}(x)\right) F_{k}^{2}(y) \mathrm{d} y\right| \\
& \lesssim 2^{-j r+k r} \int_{\mathbb{R}^{n}}\left|2^{j n} \psi^{\vee}\left(\frac{x-y}{2^{-j}}\right)\right| \frac{|x-y|^{r}}{2^{-j r}}\left|F_{k}^{2}(y)\right| \mathrm{d} y \\
& \lesssim 2^{-j r+k r} \mathcal{M}\left(\left|F_{k}^{2}\right|\right)(x),
\end{aligned}
$$

for $j>0$, where $\mathcal{M}$ is the Hardy-Littlewood maximal function. For $j=0$ this follows from the $L^{\infty}$-boundedness of $\psi_{0}(D)$ and the first estimate of (70). Therefore using the $L^{2}$-boundedness of Hardy-Littlewood maximal function, Proposition 2.16 (on $T_{\sigma_{\alpha, k}}^{\varphi}$ ) and the commutator estimate above, we obtain

$$
\begin{aligned}
\left\|\sum_{k=1}^{\infty}\left[\psi_{j}(D), M_{k}\right] F_{k}^{2}\right\|_{L^{2}\left(\mathbb{R}^{n}\right)}^{2} & \lesssim 4^{-j r} \sum_{0<|\alpha|<N_{1}}\left(\sum_{k=1}^{\infty} 2^{k(r-\varepsilon)}\left\|T_{\sigma_{\alpha, k}}^{\varphi} f_{k}\right\|_{L^{2}\left(\mathbb{R}^{n}\right)}\right)^{2} \\
& \lesssim 4^{-j r}\left(\sum_{k=1}^{\infty} 2^{k(r-\varepsilon)}\left\|f_{k}\right\|_{L^{2}\left(\mathbb{R}^{n}\right)}\right)^{2} .
\end{aligned}
$$

Hence, if $r \in(s, s+\varepsilon)$, by Cauchy-Schwarz's inequality we obtain

$$
\begin{aligned}
& \sum_{j=0}^{\infty} 4^{j s}\left\|\sum_{k=1}^{\infty}\left[\psi_{j}(D), M_{k}\right] F_{k}^{2}\right\|_{L^{2}\left(\mathbb{R}^{n}\right)}^{2} \\
& \quad \leq \sum_{j=0}^{\infty} 4^{j(s-r)}\left(\sum_{k=1}^{\infty} 2^{k(r-\varepsilon)} 2^{-k s} 2^{k s}\left\|f_{k}\right\|_{L^{2}\left(\mathbb{R}^{n}\right)}\right)^{2} \\
& \quad \leq \sum_{j=0}^{\infty} 4^{j(s-r)}\left(\sum_{k=1}^{\infty} 4^{k(r-\varepsilon-s)}\right)\left(\sum_{k=1}^{\infty} 4^{k s}\left\|f_{k}\right\|_{L^{2}\left(\mathbb{R}^{n}\right)}^{2}\right) \\
& \approx\|f\|_{H^{s}\left(\mathbb{R}^{n}\right)}^{2} .
\end{aligned}
$$

Thus putting this, (82) and (84) together, we obtain

$$
\left\|\sum_{k=1}^{\infty} M_{k} F_{k}^{2}\right\|_{H^{s}\left(\mathbb{R}^{n}\right)} \lesssim\|f\|_{H^{s}\left(\mathbb{R}^{n}\right)}
$$


We note that since we assumed that $s>0$ and $r \in(s, s+\varepsilon)$, and since $\varepsilon \in(0,1 / 2)$, these force $s$ to belong to the interval $\max (0, r-1 / 2)<s<r$, which is the claimed range of $s$ in the proposition.

Step 4 - Analysis of $\sum_{k=1}^{\infty} M_{k}(x) F_{k}^{3}(x)$

Finally we turn to the Sobolev boundedness of the term

$$
\sum_{k=1}^{\infty} M_{k}(x) F_{k}^{3}(x) .
$$

Once again, using the definition of $F_{k}^{3}$ above, we have

$$
\begin{aligned}
\psi_{j}(D) M_{k}(x) F_{k}^{3}(x)= & 2^{-k \varepsilon N_{1}} M_{k}(x) \psi_{j}(D) T_{r_{k}}^{\varphi} f_{k}(x) \\
& +2^{-k \varepsilon N_{1}}\left[\psi_{j}(D), M_{k}\right] T_{r_{k}}^{\varphi} f_{k}(x)
\end{aligned}
$$

The commutator term can be treated as term III in Step 3, since we can choose arbitrarily fast decay in $k$. After taking the sum in $k$ of the first term, using (70), then taking the $L^{2}$-norm, multiplying with $4^{j s}$ and then taking the $\ell^{2}$-norm in $j$ one has the estimate

$$
\begin{aligned}
& \sum_{k=1}^{\infty} 2^{-k \varepsilon N_{1}}\left(\sum_{j=0}^{\infty}\left\|2^{j s} M_{k}(x) \psi_{j}(D) T_{r_{k}}^{\varphi} f_{k}(x)(x)\right\|_{L^{2}\left(\mathbb{R}^{n}\right)}^{2}\right)^{1 / 2} \\
& \lesssim \sum_{k=1}^{\infty} 2^{-k \varepsilon N_{1}}\left(\sum_{j=0}^{\infty}\left\|2^{j s} \psi_{j}(D) T_{r_{k}}^{\varphi} f_{k}(x)(x)\right\|_{L^{2}\left(\mathbb{R}^{n}\right)}^{2}\right)^{1 / 2} \\
& \lesssim \sum_{k=1}^{\infty} 2^{-k \varepsilon N_{1}}\left\|T_{r_{k}}^{\varphi} f_{k}(x)\right\|_{H^{s}\left(\mathbb{R}^{n}\right)} \lesssim \sum_{k=1}^{\infty} 2^{-k \varepsilon N_{1}}\|f\|_{H^{s}\left(\mathbb{R}^{n}\right)} \lesssim\|f\|_{H^{s}\left(\mathbb{R}^{n}\right)},
\end{aligned}
$$

where we have also used Lemma 2.17.

Step 5 - Analysis of the case $\boldsymbol{k}=0$

We have, using the second estimate in (70), a similar argument as in (85), and the Sobolev-boundedness result in Lemma 2.17

$$
\begin{aligned}
& \left(\sum_{j=0}^{\infty} 4^{j s}\left\|\psi_{j}(D) M_{0} T_{1}^{\varphi} f_{0}\right\|_{L^{2}\left(\mathbb{R}^{n}\right)}^{2}\right)^{1 / 2} \\
& \leq\left(\sum_{j=0}^{\infty} 4^{j s}\left\|M_{0} \psi_{j}(D) T_{1}^{\varphi} f_{0}\right\|_{L^{2}\left(\mathbb{R}^{n}\right)}^{2}\right)^{1 / 2} \\
& \quad+\left(\sum_{j=0}^{\infty} 4^{j s}\left\|\left[\psi_{j}(D), M_{0}\right] T_{1}^{\varphi} f_{0}\right\|_{L^{2}\left(\mathbb{R}^{n}\right)}^{2}\right)^{1 / 2}
\end{aligned}
$$




$$
\begin{aligned}
& \lesssim\left\|T_{1}^{\varphi} f_{0}\right\|_{H^{s}\left(\mathbb{R}^{n}\right)}+\left(\sum_{j=0}^{\infty} 4^{j(s-r)}\left\|T_{1}^{\varphi} f_{0}\right\|_{L^{2}\left(\mathbb{R}^{n}\right)}^{2}\right)^{1 / 2} \\
& \lesssim\left\|f_{0}\right\|_{H^{s}\left(\mathbb{R}^{n}\right)}+\left\|f_{0}\right\|_{L^{2}\left(\mathbb{R}^{n}\right)} \lesssim\|f\|_{H^{s}\left(\mathbb{R}^{n}\right)} .
\end{aligned}
$$

As a corollary we obtain Theorem 2 in the introduction of the paper, namely

Corollary 7.2 Let $a \in S_{1,1}^{0}\left(\mathbb{R}^{n}\right)$ and $\varphi$ be an SND phase function in the class $\Phi^{2}$. Then for $s>0$ the FIO $T_{a}^{\varphi}$ is bounded from the Sobolev space $H^{s}\left(\mathbb{R}^{n}\right)$ to $H^{s}\left(\mathbb{R}^{n}\right)$.

Proof Note that by Proposition 7.1 we have the $H^{s}$-boundedness of $T_{a}^{\varphi}$ with $a(x, \xi) \in$ $C_{*}^{r} S_{1,1}^{0}\left(\mathbb{R}^{n}\right)$, for $\max (0, r-1 / 2)<s<r$. Moreover it is well-known that $S_{1,1}^{0}\left(\mathbb{R}^{n}\right) \subset$ $C_{*}^{r} S_{1,1}^{0}\left(\mathbb{R}^{n}\right)$ for all $r>0$, see $[3,19]$. Therefore the arbitrariness of $r$ would then yield the desired result for amplitudes in $S_{1,1}^{0}\left(\mathbb{R}^{n}\right)$.

Acknowledgements The authors are indebted to the referees for their meticulous reading of the manuscript, and for suggestions that improved the overall presentation of the paper. In particular, we are grateful for comments concerning one of our initial results, which prompted us to improve it further and transform it to a much better one.

Funding Open access funding provided by Uppsala University.

Open Access This article is licensed under a Creative Commons Attribution 4.0 International License, which permits use, sharing, adaptation, distribution and reproduction in any medium or format, as long as you give appropriate credit to the original author(s) and the source, provide a link to the Creative Commons licence, and indicate if changes were made. The images or other third party material in this article are included in the article's Creative Commons licence, unless indicated otherwise in a credit line to the material. If material is not included in the article's Creative Commons licence and your intended use is not permitted by statutory regulation or exceeds the permitted use, you will need to obtain permission directly from the copyright holder. To view a copy of this licence, visit http://creativecommons.org/licenses/by/4.0/.

\section{References}

1. Álvarez, J., Hounie, J.: Estimates for the kernel and continuity properties of pseudo-differential operators. Ark. Mat. 28, 1-22 (1990)

2. Bony, J.M.: Calcul symbolique et propagation des singularités pour les équations aux dérivées partielles non linéaires. Ann. Sci. École Norm. Sup. 4(14), 209-246 (1981)

3. Bourdaud, G.: Une algèbre maximale d'opérateurs pseudo-différentiels. Comm. Partial Differential Equations 13, 1059-1083 (1988)

4. Castro, A.J., Israelsson, A., Staubach, W., Yerlanov, M.: Regularity properties of Schrödinger integral operators and general oscillatory integrals. (arXiv:1912.08316)

5. Coifman, R.R., Meyer, Y.: Au delà des opérateurs pseudo-différentiels. Astérisque, vol. 57. Société Mathématique de France, Paris (1978)

6. Coifman, R.R., Weiss, G.: Extensions of Hardy spaces and their use in analysis. Bull. Amer. Math. Soc. 83, 569-645 (1977)

7. Cordero, E., Nicola, F., Rodino, L.: Boundedness of Fourier integral operators on $\mathscr{F} L^{p}$ spaces. Trans. Amer. Math. Soc. 361, 6049-6071 (2009)

8. Cordero, E., Nicola, F., Rodino, L.: On the global boundedness of Fourier integral operators. Ann. Global Anal. Geom. 38, 373-398 (2010)

9. Coriasco, S., Ruzhansky, M.: On the boundedness of Fourier integral operators on $L^{p}\left(\mathbf{R}^{n}\right)$. C. R. Math. Acad. Sci. Paris 348, 847-851 (2010) 
10. Coriasco, S., Ruzhansky, M.: Global $L^{p}$ continuity of Fourier integral operators. Trans. Amer. Math. Soc. 366, 2575-2596 (2014)

11. Dos Santos Ferreira, D., Staubach, W.: Global and local regularity of Fourier integral operators on weighted and unweighted spaces, Mem. Amer. Math. Soc., 229 (2014), pp. xiv+65

12. Fefferman, C.: A note on spherical summation multipliers. Israel J. Math. 15, 44-52 (1973)

13. Hassell, A., Portal, P., Rozendaal, J.: Off-singularity bounds and hardy spaces for fourier integral operators. To appear in Trans. Amer. Math. Soc. 2020, arXiv:1802.05932v4

14. Hörmander, L. Pseudo-differential operators and hypoelliptic equations. Singular integrals: (Proc. Sympos. Pure Math., Vol. X), Amer. Math. Soc., Providence, R.I., (1967), pp. 138-183

15. Hörmander, L.: Lectures on nonlinear hyperbolic differential equations. Mathématiques \&amp; Applications (Berlin), vol. 26. Springer-Verlag, Berlin (1997)

16. Israelsson, A., Rodríguez-López, S., Staubach, W.: Local and global estimates for hyperbolic equations in Besov-Lipschitz and Triebel-Lizorkin spaces. To appear in Analysis \& PDE 2020 (arXiv: $1802.05932 v 4)$

17. Kenig, C.E., Staubach, W.: $\Psi$-pseudodifferential operators and estimates for maximal oscillatory integrals. Studia Math. 183, 249-258 (2007)

18. Melrose, R.B., Taylor, M.E.: The radiation pattern of a diffracted wave near the shadow boundary. Comm. Partial Differential Equations 11, 599-672 (1986)

19. Meyer, Y.: Régularité des solutions des équations aux dérivées partielles non linéaires (d'après J.-M. Bony), in Bourbaki Seminar, Vol. 1979/80, vol. 842 of Lecture Notes in Math., Springer, Berlin-New York, 1981, pp. 293-302

20. Rodríguez-López, S., Rule, D., Staubach, W.: A Seeger-Sogge-Stein theorem for bilinear Fourier integral operators. Adv. Math. 264, 1-54 (2014)

21. Rodríguez-López, S., Staubach, W.: Estimates for rough Fourier integral and pseudodifferential operators and applications to the boundedness of multilinear operators. J. Funct. Anal. 264, 2356-2385 (2013)

22. Ruzhansky, M., Sugimoto, M.: A local-to-global boundedness argument and Fourier integral operators. J. Math. Anal. Appl. 473, 892-904 (2019)

23. Seeger, A., Sogge, C.D., Stein, E.M.: Regularity properties of Fourier integral operators. Ann. Math. 2(134), 231-251 (1991)

24. Stein, E.M.: Harmonic analysis: real-variable methods, orthogonality, and oscillatory integrals. Princeton Mathematical Series, vol. 43. Princeton University Press, Princeton, NJ (1993)

25. Taylor, M.E.: Tools for PDE, vol. 81 of Mathematical Surveys and Monographs, American Mathematical Society, Providence, RI, 2000. Pseudodifferential operators, paradifferential operators, and layer potentials

26. Triebel, H.: Theory of function spaces. Mathematik und ihre Anwendungen in Physik und Technik [Mathematics and its Applications in Physics and Technology], vol. 38. Akademische Verlagsgesellschaft Geest \& Portig K.-G, Leipzig (1983)

27. Triebel, H.: Theory of function spaces IV., vol. 107 of Monographs in Mathematics, Birkhäuser Verlag, Basel, (2020)

Publisher's Note Springer Nature remains neutral with regard to jurisdictional claims in published maps and institutional affiliations. 